ANL-6779

Engineering and

Equipment

(TID-4500, 24th Ed.)

AEC Research and

Development Report

\author{
ARGONNE NATIONAL LABORATORY \\ 9700 South Cass Avenue \\ Argonne, Illinois 60440
}

\author{
TWO-PHASE CRITICAL FLOW WITH APPLICATION \\ TO LIQUID-METAL SYSTEMS \\ (Mercury, Cesium, Rubidium, Potassium, \\ Sodium, and Lithium) \\ by \\ Hans K. Fauske \\ Reactor Engineering Division, ANL \\ and \\ Associated Midwest Universities
}

October 1963

This report is one of a series that describes heattransfer and fluid-flow studies performed at Argonne under a program sponsored jointly by the Associated Midwest Universities and the Argonne National Laboratory.

The earlier reports in this series are ANL-6625, ANL-6667, ANL-6674, ANL-6710, ANL-6734, ANL6738, ANL-6754, and ANL-6755.

Operated by The University of Chicago under

Contract $W-31-109$-eng-38 with the

U. S. Atomic Energy Commission 


\section{DISCLAIMER}

This report was prepared as an account of work sponsored by an agency of the United States Government. Neither the United States Government nor any agency Thereof, nor any of their employees, makes any warranty, express or implied, or assumes any legal liability or responsibility for the accuracy, completeness, or usefulness of any information, apparatus, product, or process disclosed, or represents that its use would not infringe privately owned rights. Reference herein to any specific commercial product, process, or service by trade name, trademark, manufacturer, or otherwise does not necessarily constitute or imply its endorsement, recommendation, or favoring by the United States Government or any agency thereof. The views and opinions of authors expressed herein do not necessarily state or reflect those of the United States Government or any agency thereof. 


\section{DISCLAIMER}

Portions of this document may be illegible in electronic image products. Images are produced from the best available original document. 
TABLE OF CONTENTS

Page

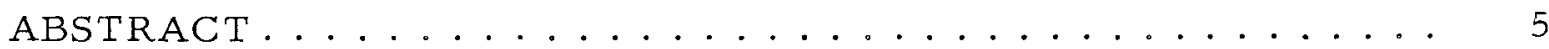

I. INTRODUCTION. .......................... 5

II. EXISTING DATA FOR STEAM-WATER CRITICAL FLOW IN PIPES ....................... 7

III. MODELS OF CRITICAL TWO-PHASE FLOW........... 9

A. Homogeneous-flow Mode1................. 9

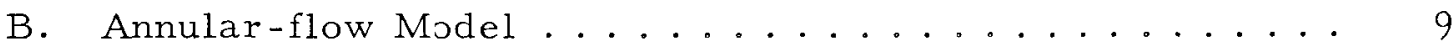

IV. COMPARISON OF FLOW MODELS AND EXPERIMENTAL DATA FOR CRITICAL FLOW OF STEAM-WATER . . . . . . 12

V. APPLICATION TO LIQUID-METAL SYSTEMS. . . . . . . . 18 APPENDICES

APPENDIX A - Fortran Listing Program.......... 22

APPENDIX B - Computer Output Print Outs of Critical Flow for Liquid Metals ............... 23

ACKNOWLEDGEMENT . . . . . . . . . . . . . . 48

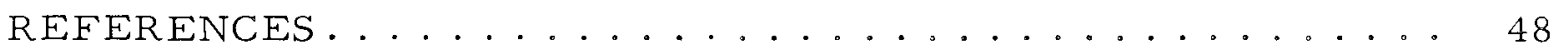

NOMENCLATURE . . . . . . . . . . . . . . . . . . 49 


\section{LIST OF FIGURES}

No.

Title

Page

1. Comparison between Various Two-phase Critical Flow

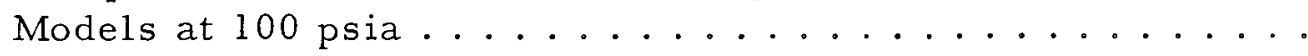

2. Comparison between Various Two-phase Critical Flow

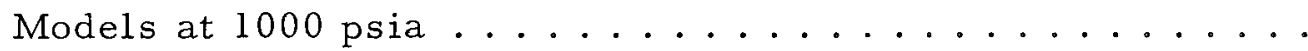

3. Comparison between Various Two-phase Critical Flow Models at $2000 \mathrm{psia} \ldots \ldots \ldots \ldots \ldots$

4. Theoretical Predictions of Critical-flow Rates for SteamWater from the Fauske Theory ...............

5. Comparison of Combined Data from References 2, 4, and 6 with the Fauske Model at 2 per cent Quality .........

6. Comparison of Combined Data from References 2, 4, and 6 with the Fauske Model at 5-15 per cent Quality ........

7. Comparison of Combined Data from References 2, 4, and 6 with the Fauske Model at 15-25 per cent Quality ........

8. Comparison of Combined Data from References 2, 4, and 6 with the Fauske Model at 25-35 per cent Quality ........

9. Comparison of the New Zealand Data with the Fauske Model at $20 \mathrm{psia} \ldots \ldots \ldots \ldots \ldots \ldots \ldots$

10. Comparison of the New Zealand Data with the Fauske Model

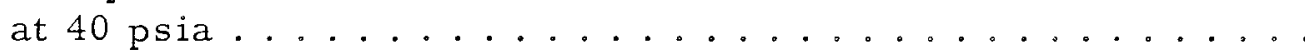

11. Theoretical Prediction of Void Fraction for Rubidium under Critical-flow Conditions . . . . . . . . . . . .

12. Theoretical Prediction of Slip Ratio for Rubidium under

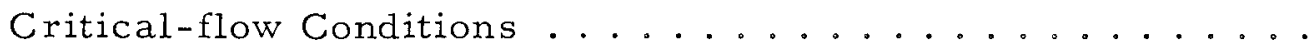

13. Theoretical Prediction of Two-phase Critical-flow Rates

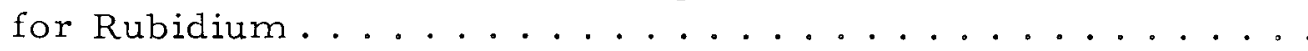

14. Comparison of Flow Rates Calculated from Fauske Theory and Homogeneous-equilibrium Theory for Rubidium at 5 psia. 


\section{LIST OF TABLES}

No.

Title

Page

1. Computer Input Data for Rubidium. . . . . . . . . . . . 24

2. Critical Flow Data for Rubidium ............ 25-27

3. Computer Input Data for Lithium............... 28

4. Critical Flow Data for Lithium ............. 29-30

5. Computer Input Data for Mercury ............ 31

6. Critical Flow Data for Mercury ................ 32-35

7. Computer Input Data for Potassium ............. 36

8. Critical Flow Data for Potassium ............ 37-39

9. Computer Input Data for Cesium ............... 40

10. Critical Flow Data for Cesium .............. 41-43

11. Computer Input Data for Sodium ............... 44

12. Critical Flow Data for Sodium .............. . 45-47 


\title{
TWO-PHASE CRITICAL FLOW WITH APPLICATION \\ TO LIQUID - METAL SYSTEMS \\ (Mercury, Cesium, Rubidium, Potassium, \\ Sodium, and Lithium)
}

by

Hans K. Fauske

\begin{abstract}
Existing data and models for critical two-phase flow of steam-water mixtures are examined. One model in particular shows considerable success in predicting the phenomena of critical flow. A Fortran program for an IBM-704 digital computer, for the application of this model to predict void fraction, slip ratio, and critical flow rates to the flashing flow for various liquid-vapor metal systems in pipes when the conditions are such that critical flow may be experienced, was developed. Calculations have been made for the following liquid-metal systems: mercury (temperature range, 450$1600^{\circ} \mathrm{F}$ ), cesium (temperature range, $500-2300^{\circ} \mathrm{F}$ ), rubidium (temperature range, $500-2300^{\circ} \mathrm{F}$ ), potassium (temperature range, $900-2500^{\circ} \mathrm{F}$ ), sodium (temperature range, $950-2500^{\circ} \mathrm{F}$ ), and lithium (temperature range, 2000-3500 $\mathrm{F}$ ). The vapor fraction or quality ranges from 0 to $100 \%$ 。

It is shown that the critical flow rates for liquidmetal systems calculated from the best model describing steam-water data are considerably higher in the low-quality region than predicted from a so-called "homogeneous flow model." This is explained by the large slippage between the liquid and the vapor phases, because the ratio of the densities of the liquid state to the vapor state is usually large for metallic fluids.
\end{abstract}

\section{INTRODUCTION}

Although steam-water systems have been utilized for a long time as a coolant, the higher heat fluxes experienced in current reactors, missiles, rockets, and space applications require the use of coolants with more attractive thermodynamic properties than water. For this reason the problems of two-phase flow and of heat transfer to liquid metals have recently received a great deal of attention. Although considerable critical 
flow data are available for water, no data exist for liquid metals. Extrapolation of a correlation built up of experimental parameters based primarily on water is dangerous because the physical properties of liquid metals differ markedly from those of water. Particularly striking for a given temperature is the occurrence of extremely high liquid-to-gas density ratios for liquid metals. However, one would be on much safer ground to utilize a model or theory with no experimental constants that explain steam-water data in order to estimate critical flow rates for liquid-vapor metal systems.

In the present report the best available theory is utilized to calculate void fractions, slip ratios, and flow rates for liquid-vapor metal systems in pipes under critical flow conditions. It is postulated that the mechanism causing critical flow for a liquid-vapor metal system is the same as for a steam-water system. Therefore, the only apparent dissimilarity between the two systems is the respective physical properties.

In the two sections that follow, existing data and models for critical two-phase flow of steam water are summarized. 


\section{EXISTING DATA FOR STEAM-WATER CRITICAL FLOW IN PIPES}

The primary purpose of this section is to summarize the present available data sources for one-component, two-phase critical flow of water in constant-area tubes. Only works which have specifically treated the phenomenon of critical flow will be mentioned.

Data of Isbin et al.

Isbin et al. ${ }^{(1)}$ in 1957 reported on the results of one of the first nonclassified studies undertaken explicitly to measure critical flows of steamwater mixtures over a wide range of qualities. Critical exit pressures from 4 to 43 psia were obtained, and the quality ranged from saturated vapor to $1 \mathrm{w} / 0$ vapor. Discharges were measured from $\frac{1}{4}-, \frac{1}{2}-, \frac{3}{4}-$, and $1-$ in. pipes and from annuli of intermediate cross-sectional areas. The experimental mass flow rates were found always to be greater than the values calculated on the basis of a homogeneous-flow model, discussed in the section that follows. Several empirical methods for correlating the data were determined, and comparisons were presented of the predictions of several semianalytical flow models. All of the se models showed substantial deviations from the measured flow rates at small qualities (less than $20 \%$ ).

Faletti's Data

In 1959, Faletti $^{(2)}$ presented one of the most extensive studies carried out on two-phase, one-component critical flow. The flow of steamwater mixtures was studied in concentric annuli having center rods of 0.187 - and 0.375 -in. OD. Critical throat pressures ranged from 26 to 106 psia, and qualities ranged from 0.1 to $97.5 \%$. The data were correlated by plotting the ratio of the observed mass velocity to the theoretical mass velocity (homogeneous equilibrium theory) versus the quality. This is the same approach as was used by Isbin. Also, Faletti found his measured flow rates to be considerably higher than predicted from the homogeneous-flow theory. This work was recently and excellently summarized by Faletti and Moulton. (3)

Fauske's Data

In 1961, Fauske ${ }^{(4)}$ presented critical-flow data at considerably higher pressures than previously reported. Critical two-phase, steamwater flows were measured over a range of quality from 0.01 to 1.0 , total flows from 500 to $4200 \mathrm{lb} / \mathrm{sec}-\mathrm{ft}^{2}$, pressures from 40 to $360 \mathrm{psia}$, and with pipe diameters of $0.125-, 0.269-$, and 0.5-in. ID. A theory was developed for the two-phase, critical-flow phenomenon, and extensive comparisons of predicted with experimental values showed substantial agreement. This theory will be derived in the section that follows. Details of this work are reported elsewhere. (5) 
Zaloudek's Data

Also in 1961 Zaloudek(6) presented his data on two-phase critical flow in pipes. Experiments were limited to circular full-bore cross sections of $0.520-$ and 0.625 -in. diameter, and lengths up to $4 \mathrm{ft}$. Test-section exit qualities from 0.4 to $99 \%$ and critical mass velocities between $10^{2}$ and $10^{3} \mathrm{lb} / \mathrm{sec}-\mathrm{ft}^{2}$ were investigated. Corresponding critical pressures ranged from 40 to 110 psia. The results obtained were correlated by employing the conventional technique of comparing results with theoretical predictions of the homogeneous-flow model. Also in this investigation the measured flow rates were considerably higher than predicted from the homogeneous flow theory.

James' Data

In 1962 , James $(7)$ presented critical-flow data at considerably larger pipe sizes than previously reported. Steam and steam-water flashing flow were used to obtain critical discharge pressures for pipes over the stagnation enthalpy range from 230 to $1200 \mathrm{Btu} / 1 \mathrm{~b}$ and for the critical pressure range from 14 to 64 psia. Pipes used were 3,6 , and 8 in. in diameter. A method similar to that of Isbin et al. (1) was used to correlate the data.

For experimental details and procedures of these works, the reader is referred to the respective reference. In the section that follows various flow models are derived and compared with the above-mentioned data.

The following major conclusions can be extracted from these works briefly summarized above:

1. Each set of experimental data seems to agree fairly well with the others. No irregularities appear, although experimental techniques are not always the same.

2. No geometry or upstream-mixing effects appear. Hence, for the geometries examined, the critical-flow phenomenon can be described by the local conditions at the exit of the test section only.

3. In all cases the measured critical-flow rates were higher than values calculated from the homogeneous-flow model, particularly in the low-quality region.

4. A theoretical model has been developed that satisfactorily describes the above-mentioned data (see next section). 


\section{MODELS OF CRITICAL TWO-PHASE FLOW}

Before discussing individual flow models, the basic equations of change governing a two-phase flow system will be written:

$$
\begin{aligned}
& \frac{\partial}{\partial t}\left(\sum_{i=1}^{2} \alpha_{i} \rho_{i}\right)+\frac{\partial}{\partial x}\left(\sum_{i=1}^{2} \alpha_{i} \rho_{i} \bar{u}_{i}\right)=0 \\
& \frac{\partial}{\partial t}\left(\sum_{i=1}^{2} \alpha_{i} \rho_{i} \bar{u}_{i}\right)+\frac{\partial}{\partial x}\left(\sum_{i=1}^{2} \alpha_{i} \rho_{i} \bar{u}_{i}^{2}\right)+\frac{\partial p}{\partial x}+\frac{\partial \tau_{2}}{\partial \mathbf{x}}+\sum_{i=1}^{2} \alpha_{i} \rho_{i} g=0
\end{aligned}
$$

where $\bar{u}_{i}$ is the average velocity of the $i^{\text {th }}$ phase in the $x$ direction.

Equations (1) and (2) will now be utilized to derive various simpli.fied models.

\section{A. Homogeneous-flow Mode1}

In this theory, the momentum and continuity equations are applied to a homogeneous mixture in which the average velocities of liquid and steam are equal. Furthermore, steady-state flow is assumed, and the critical-flow condition is defined under the restraints that the change in mass flow rate with respect to the pressure is zero at constant entropy. Under these conditions, Eqs. (1) and (2) reduce to the following equations which define the critical mass flow rate:

$$
\begin{aligned}
G^{2} & =-g_{c}\left(\frac{d p}{d v}\right)_{s} ; \\
v & =v_{f}+v_{f g} \chi
\end{aligned}
$$

B. Annular-flow Model

An annular-flow pattern is assumed with an average liquid velocity in the annulus and an average steam velocity in the core, but different in magnitude. The process is considered isentropic with both phases in equilibrium. Under these conditions, Eqs. (1) and (2) reduce to the following equations, which define the critical mass flow rate:

$$
\begin{aligned}
\mathrm{G}^{2} & =-\mathrm{g}_{\mathrm{C}}\left(\frac{\mathrm{dp}}{\mathrm{dv}}\right)_{\mathrm{s}} ; \\
\mathrm{v} & =\frac{\chi^{2} \mathrm{v}_{\mathrm{g}}}{\alpha_{\mathrm{g}}}+\frac{(1-\chi)^{2} \mathrm{v}_{\mathrm{f}}}{1-\alpha_{\mathrm{g}}} .
\end{aligned}
$$


The unknown quantity introduced here, the void fraction $\alpha$, has indeed never been measured for steam-water systems under critical-flow conditions. It has, therefore, been the practice to utilize void-fraction correlations obtained under restricted flow conditions where normally the velocities are much smaller than pertaining to critical flow. Two of the most frequently used void-fraction correlations will be quoted here.

\section{Modified Martinelli Data for Void Fraction}

The Martinelli values of void fraction were modified and fitted to a curve at the Bettis Atomic Power Laboratory, Westinghouse Electric Corporation. (8)

\section{Modified Armand Formula for Void Fraction}

The Armand formula for void fraction, modified at the Hanford Atomic Products Operation, General Electric,(9) is as follows:

$$
\alpha_{g}=\frac{(0.833+0.167 \chi) \chi v_{g}}{(1-\chi) v_{f}+\chi v_{g}}
$$

The incorporation of these two void-fraction correlations with Eqs. (5) and (6) renders two possible solutions for the critical-flow rate.

\section{Fauske Mode1}

The following assumptions are made in the development of the model (for a more detailed discussion, see Ref. 5):

a. An average velocity exists for each phase and is different in magnitude for each phase.

b. The vapor and liquid are in equilibrium throughout the flow path.

c. Critical flow is attained when the flow rate no longer increases with decreasing static pressure:

$\frac{\mathrm{dG}}{\mathrm{dp}}=0$

d. The pressure gradient attains a finite, maximum value for a given flow rate and quality:

$$
\left|\frac{\mathrm{dp}}{\mathrm{dx}}\right|_{\mathrm{G}, \chi}=\{\operatorname{Max}\}_{\text {Finite }} .
$$


In a two-phase flow system, the pressure drop becomes a function of slip ratio besides the usual variables occurring in single-phase flow. From Eq. (9) it can be seen that all system variables are fixed except the void fraction $\alpha$. The void fraction is, in other words, one extra degree of freedom in the two-phase system. If isentropic flow is assumed for these conditions, the maximization of the flow rate is achieved by varying the void fraction $\alpha$ :

$$
\frac{\partial}{\partial \alpha_{g}}\left[\left\{\begin{array}{l}
\text { Rate of momentum } \\
\text { gain by convection }
\end{array}\right\}\right]=0 .
$$

Under these conditions Eqs. (1) and (2) reduce to the following equations defining the critical mass flow rate:

$$
\begin{aligned}
\mathrm{G}^{2} & =-\mathrm{g}_{\mathrm{c}}\left(\frac{\mathrm{dp}}{\mathrm{dv}}\right)_{\mathrm{s}} ; \\
\mathrm{v} & =\frac{\chi^{2} \mathrm{v}_{\mathrm{g}}}{\alpha_{\mathrm{g}}}+\frac{(1-\chi)^{2} \mathrm{v}_{f}}{1-\alpha_{\mathrm{g}}} ; \\
\alpha_{\mathrm{g}} & =\left[\frac{1-\chi}{\chi}\left(\frac{\mathrm{v}_{f}}{\mathrm{v}_{\mathrm{g}}}\right)^{1 / 2}+1\right]^{-1},
\end{aligned}
$$


IV. COMPARISON OF FLOW MODELS AND EXPERIMENTAL DATA FOR CRITICAL FLOW OF STEAM-WATER

Due to the extremely steep pressure gradients prevailing under critical-flow conditions, one would expect slip to occur between the liquid and vapor phases, even more so for liquid-metal than for steam-water systems. It is, therefore, to be expected that models including slip will yield results closer to the experimental findings than obtained from the homogeneous-flow model. Since void-fraction measurements have not been carried out for critical flow, various void-fraction correlations for restricted steam-water flow have been utilized. To extrapolate these to liquid-metal systems may be dangerous. A theoretical relationship for the void fraction under critical-flow conditions has been developed in the Fauske model. Because of its theoretical derivation, there appears to be no reason why this relationship should not hold for liquid-metal systems.

Nahavandi and Rashevsky, $(10)$ in describing the loss-of-coolant accident in a nuclear reactor, compared the same flow models as described in this report. Figures 1,2 , and 3 are reproduced from their report and illustrate their conclusions as quoted below:

1. The Fauske theory yields consistent results over a wide range of pressures and steam qualities encountered in the two-phase steam-water region.

2. The annular-flow theory with modified Martinelli or Armand void fractions is in good agreement with the Fauske theory for steam qualities above $10 \%$. At lower steam qualities, the calculated values of mass flow velocities by this theory become inconsistent and are not dependable. This discontinuity is caused by the inaccuracies of modified Martinelli and Armand void fractions at low steam qualities.

3. The homogeneous-model theory does not agree gene rally with the other theories except at very high steam qualities.

4. The Fauske theory appears to be the best choice for the prediction of critical flow discharge of steam-water mixtures. On the basis of this theory, a critical flow chart has been prepared.

In Fig. 4 the critical-flow solutions for the Fauske model are shown for a pressure range from 20 psia to the critical pressure, and for qualities from 0.01 to 1.0. In Fig. 5, 6, 7, 8, 9, and 10 comparisons between the Fauske model and the previously discussed data are shown. Particularly encouraging is the good agreement with the New Zealand data, which were obtained with the use of considerably larger pipe sizes than had been previously reported in the literature. 


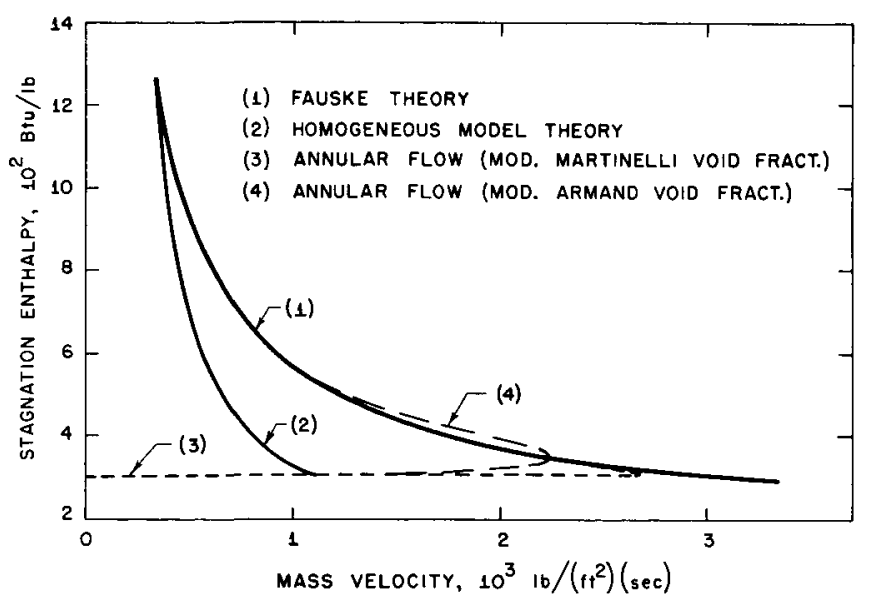

Fig. 1

Comparison between Various Two-phase Critical Flow Models at 100 psia (taken from Ref. 10)

Fig. 2

Comparison between Various Two-phase Critical Flow Models at 1000 psia (taken from Ref. 10)
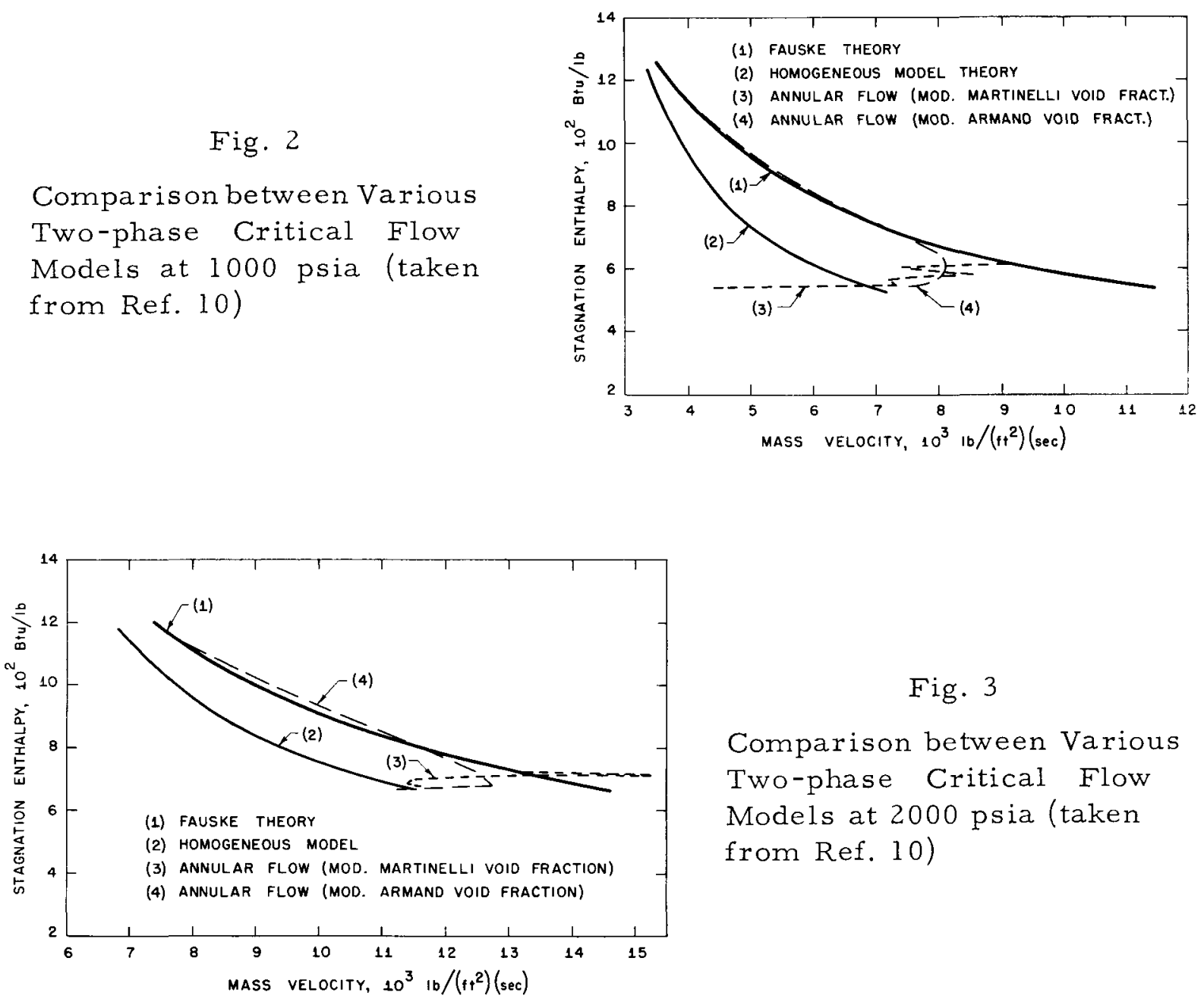

Fig. 3

Comparison between Various Two-phase Critical Flow Models at 2000 psia (taken from Ref. 10) 


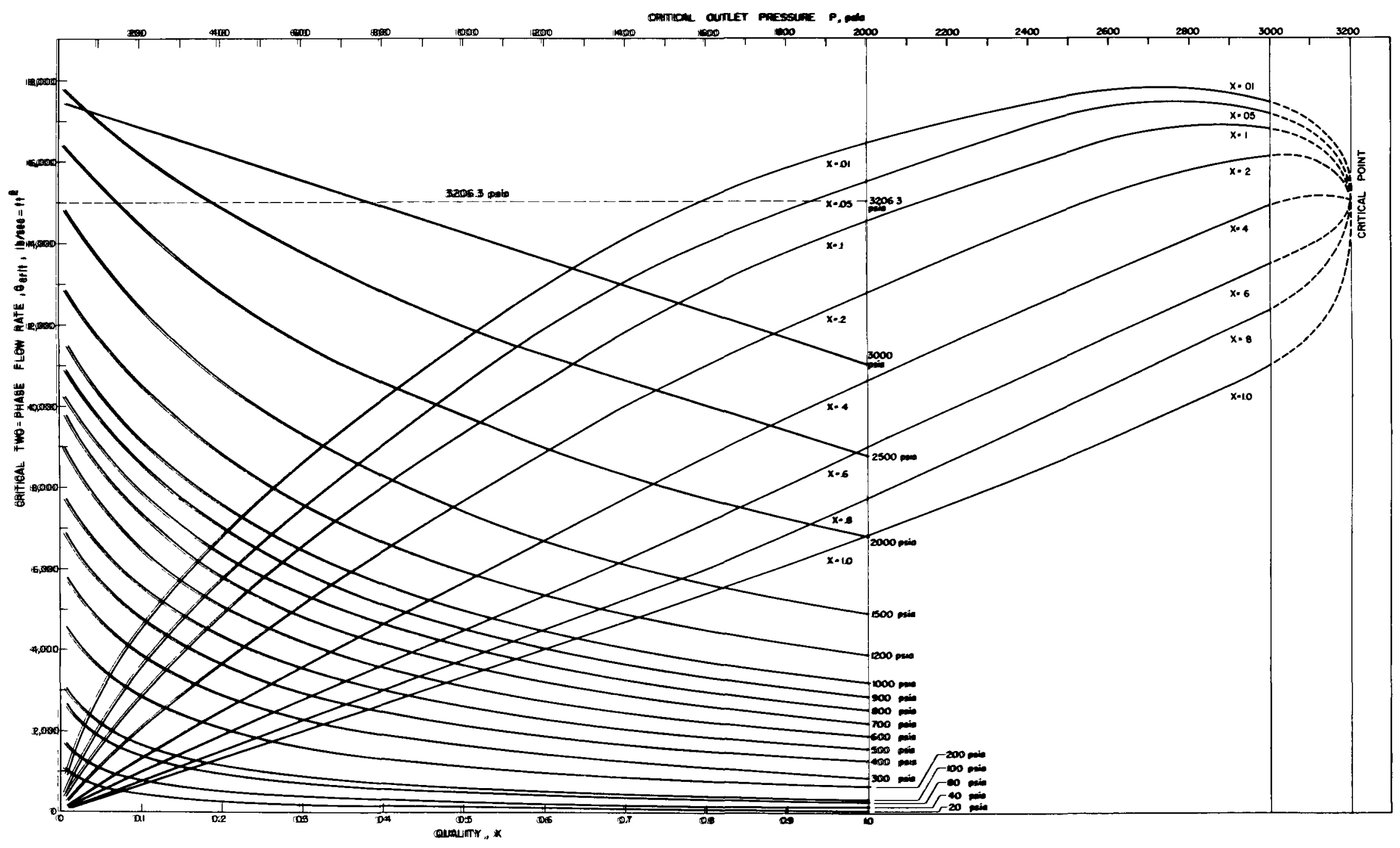

$112-2310$

Fig. 4.. Theoreticall Predictions of Critical-flow Rates for Steam-Water from the Fauske Theory 


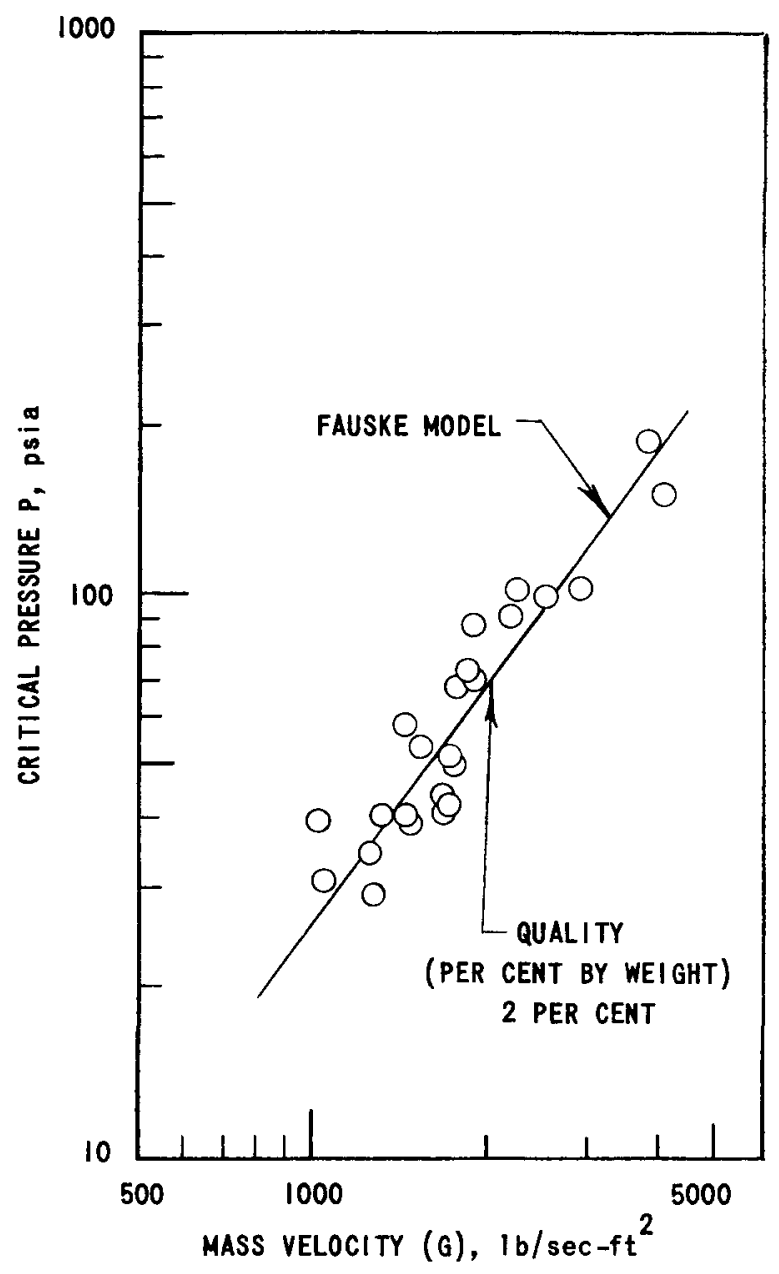

Fig. 5

Comparison of Combined Data from Refs. 2, 4, and 6 with the Fauske Model at 2 Per Cent Quality (Data points reproduced from Ref. 6)

$112-2168$

Fig. 6

Comparison of Combined Data from Refs. 2, 4, and 6 with the Fauske Model at 5-15 Per Cent Quality (Reproduced from Ref. 6)

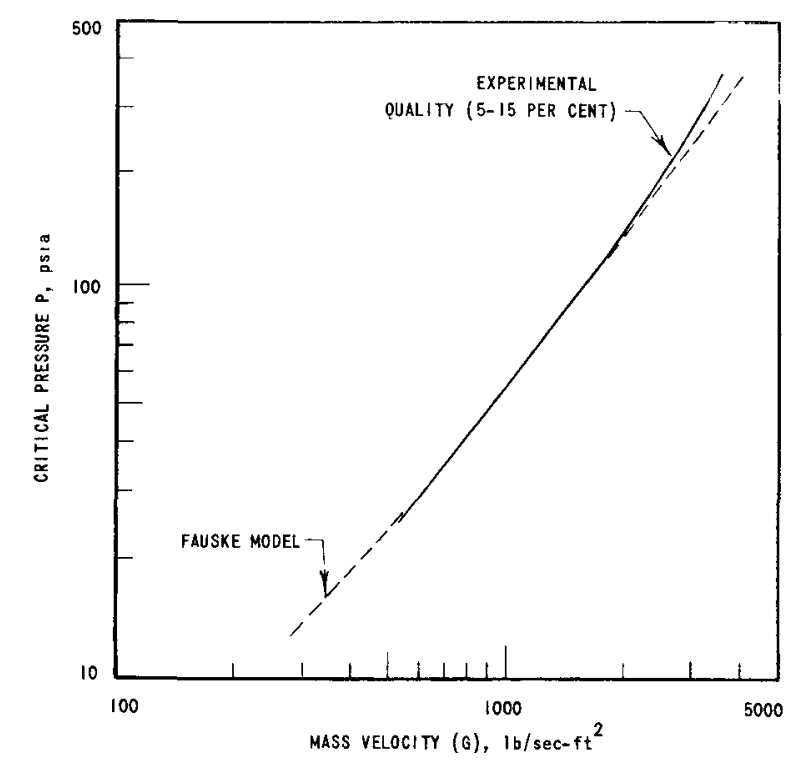

$112-2169$ 


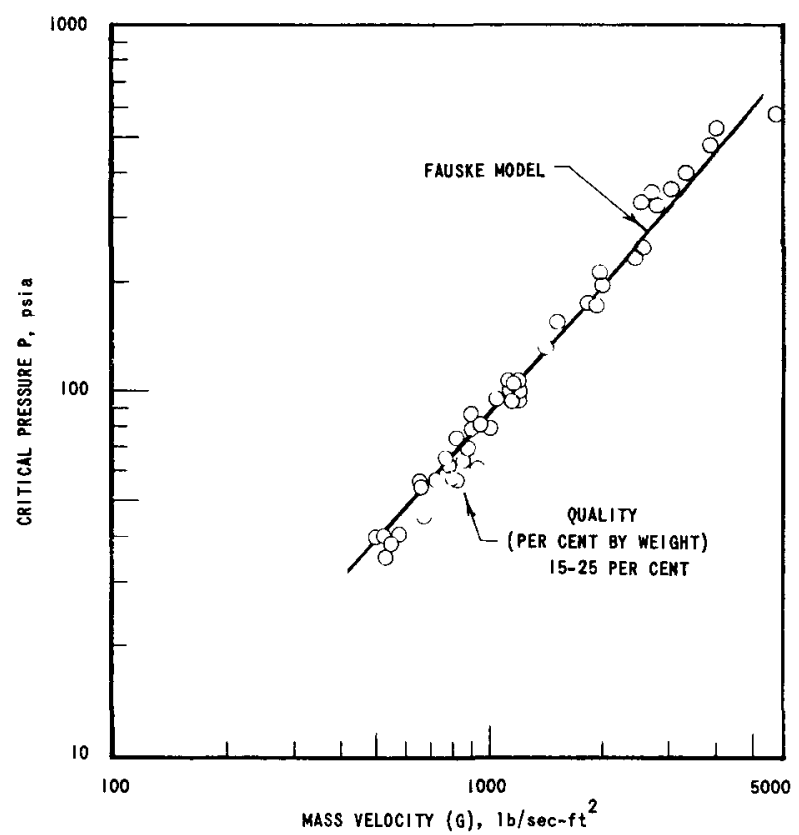

$112-2163$
Fig. 7

Comparison of Combined Data from Refs. 2, 4, and 6 with the Fauske Model at 15-25 Per Cent Quality (Data points reproduced from Ref. 6)
Fig. 8

Comparison of Combined Data from Refs. 2, 4, and 6 with the Fauske Model at 25-35 Per Cent Quality (Data points reproduced from Ref. 6)

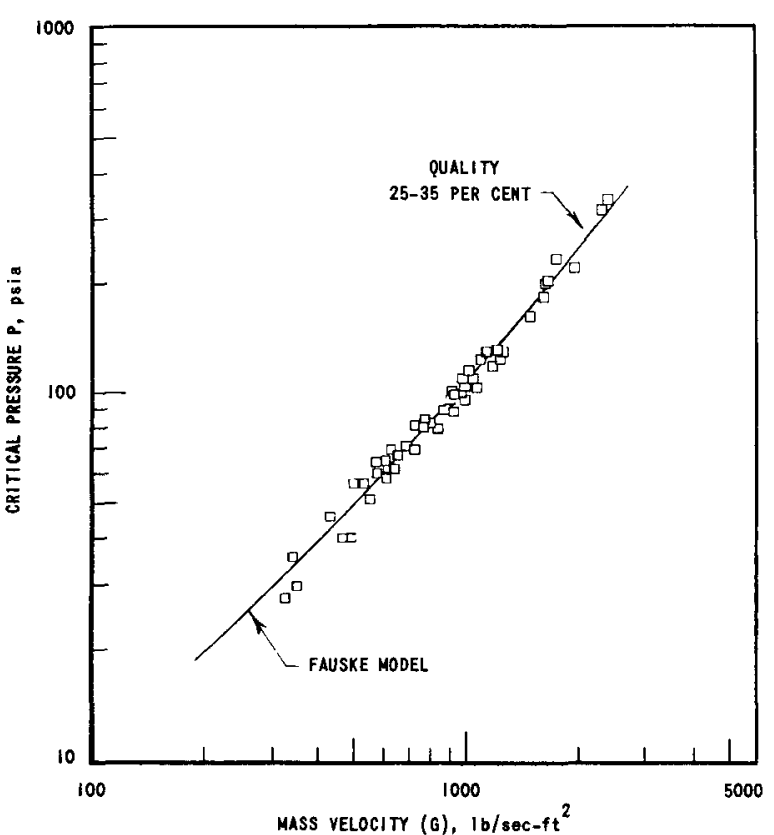

$112-2166$ 


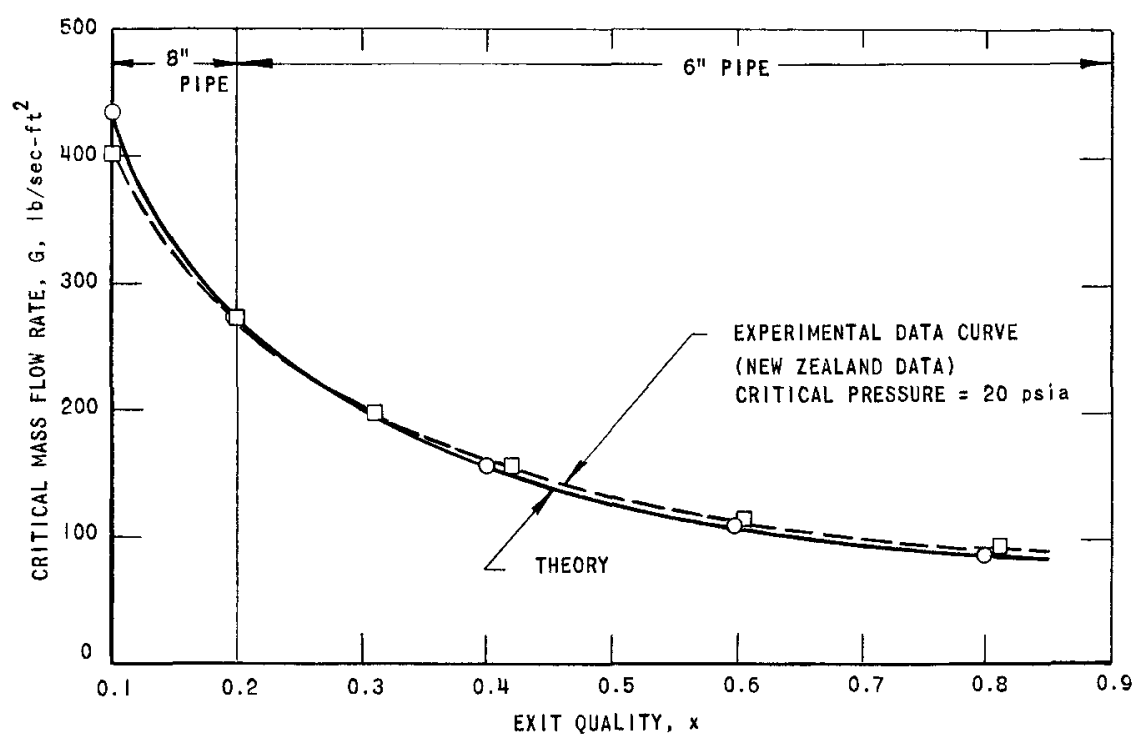

$112-2173$

Fig. 9. Comparison of the New Zealand Data with the Fauske Model at 20 psia

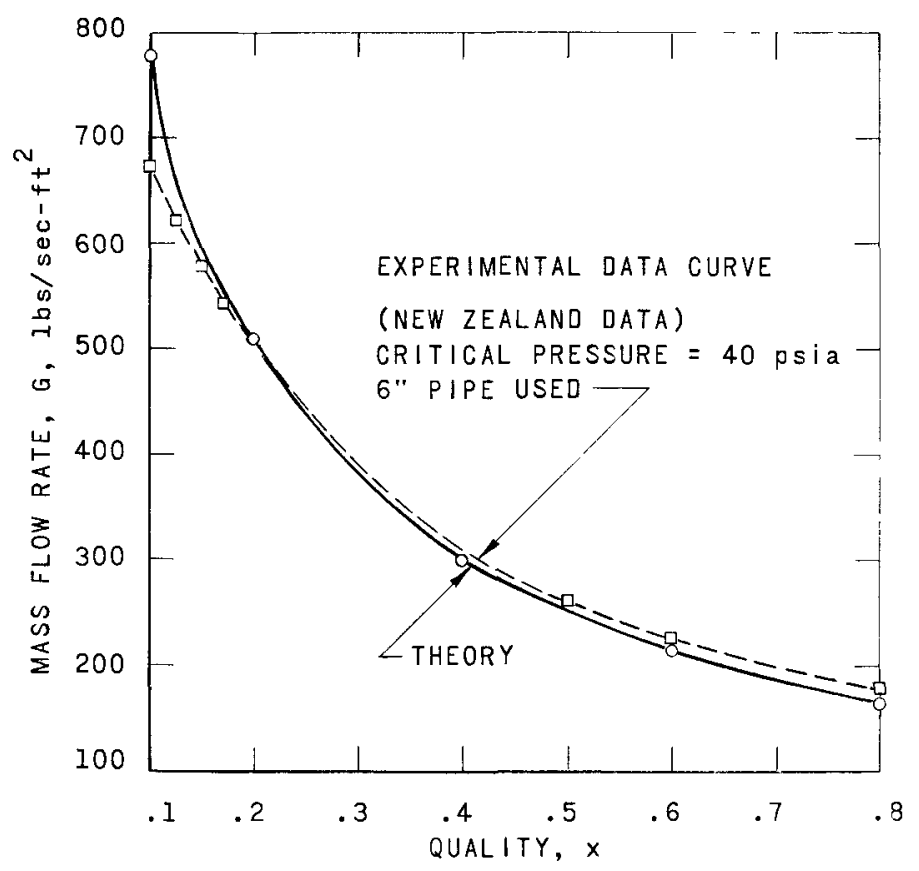

$112-2164$

Fig. 10. Comparison of the New Zealand Data with the Fauske Model at 40 psia 


\section{APPLICATION TO LIQUID-METAL SYSTEMS}

Theoretical predictions by Fauske of the two-phase critical-flow phenomenon for steam-water, have yielded such encouraging agreement with experimental data that it seems legitimate to make a similar application to the critical flow of liquid-vapor metal systems in pipes. The following liquid-metal systems were considered: mercury (temperature range, $450-1600^{\circ} \mathrm{F}$ ), cesium (temperature range, 500-2300 ${ }^{\circ} \mathrm{F}$ ), rubidium (temperature range, $500-2300^{\circ} \mathrm{F}$ ), potassium (temperature range, $900-$ $2500^{\circ} \mathrm{F}$ ), sodium (temperature range, $950-2500^{\circ} \mathrm{F}$ ), and lithium (temperature range, $2000-3500^{\circ} \mathrm{F}$ ). The vapor fraction or quality ranged from 0 to $100 \%$.

The equations as programmed on an IBM 704 computer are as follows:

$$
\begin{aligned}
& k=\left(v_{g} / v_{f}\right)^{1 / 2} ; \\
& \alpha_{\mathrm{g}}=\frac{1}{1+\mathrm{k}^{-1}[(1 / \chi)-1]} ; \\
& \frac{d \chi}{d p}=-\frac{1}{s_{g}-s_{f}}\left[(1-\chi) \frac{d s_{f}}{d p}+\chi \frac{d s_{g}}{d p}\right] ; \\
& G=\left\{\frac{-144 k g}{[(1-\chi+k \chi) \chi] \frac{d v_{g}}{d p}+\left[v_{g}(1+2 k \chi-2 \chi)+v_{f}\left(2 k \chi-2 k-2 k^{2} \chi+k^{2}\right)\right] \frac{d \chi}{d p}}\right\}_{(17)}^{1 / 2}
\end{aligned}
$$

Equation (17) is obtained by substituting Eqs. (12) and (13) into Eq. (11) and performing the differentiation with respect to pressure.

The variables used are pressure $p$, metal-vapor quality $\chi$, and mas flow rate $G$. The pressure and quality are taken as independent variables. The mass velocity is used as dependent variable and is calculated in terms of the pressure and steam quality. The computational scheme is as follows:

1. Assume a value for the pressure.

2. Calculate the slip ratio from Eq. (14).

3. Assume a value for the quality.

4. Calculate void fraction from Eq. (15).

5. Calculate $\mathrm{d} \chi / \mathrm{dp}$ from Eq. (16).

6. Calculate the mass flow rate from Eq. (17).

7. Maintaining the pressure constant, assume a new value for the steam quality, and repeat steps 4 to 7 until all the values of quality array are used. 
8. Assume a new value for the pressure and repeat steps 2 to 8 until all the values of pressure array are used.

Input data beside $p$ and $\chi$ are $v_{g}, v_{f}, s_{g}, s_{f}, d v_{g} / d p, d s_{f} / d p$, and $\mathrm{dsg} / \mathrm{dp}$. The physical and thermodynamic properties of the various liquid metals are taken from Weatherford et al. (11)

The Fortran source program listing and the output print-outs are presented in Appendices $A$ and $B$. The output print-outs consist of the input data and the calculated values for $d \chi / d p$, slip ratio $k$, void fraction $\alpha_{g}$, and critical flow rate $G$ for the various liquid metals.

In Figs. 11, 12, and 13 the computed results are shown for rubidium. Figure 11 shows how void fraction $\alpha_{g}$ varies with quality $\chi$, the pressure $p$ being taken as a parameter. Figure 12 presents the slip ratio $k$ as a function of pressure. In Fig. 13 the critical-flow rate $G$ is plotted versus the quality, with pressure as parameter. The range of quality is from 1 to $100 \%$ and that of pressure from 1 to 300 psia. These graphs indicate the various trends of the computed results and are similar for each liquid-metal system. The computed results in tabulated form may be found in Appendix B.

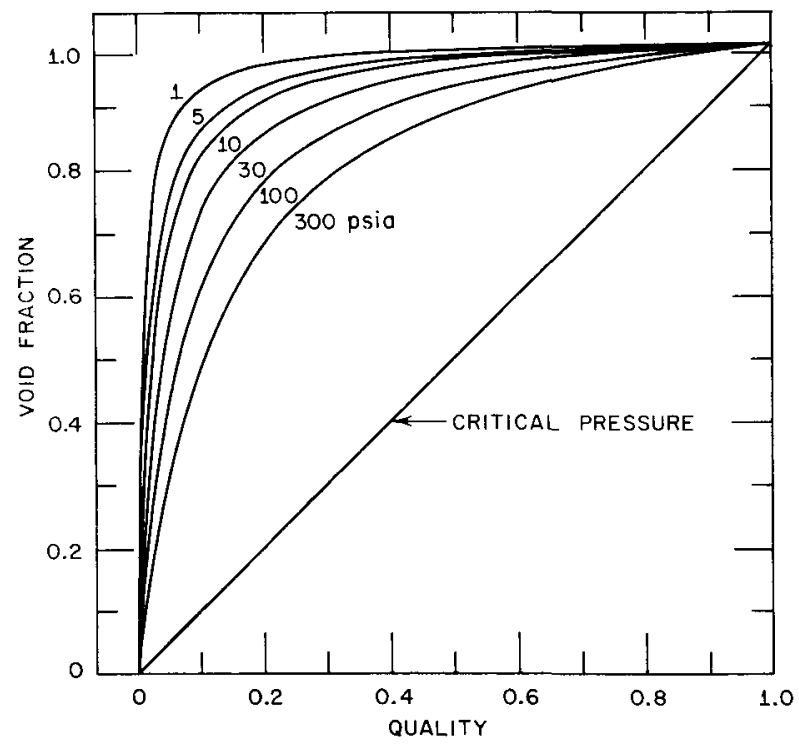

Fig. 11

Theoretical Prediction of Void Fraction for Rubidium under Critical-flow Conditions

Fig. 12

Theoretical Prediction of Slip Ratio for Rubidium under Criticalflow Conditions

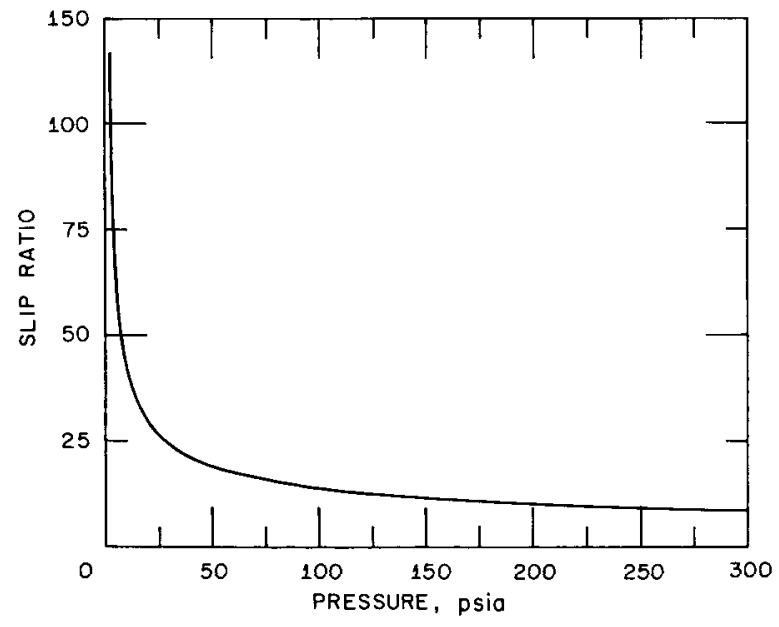




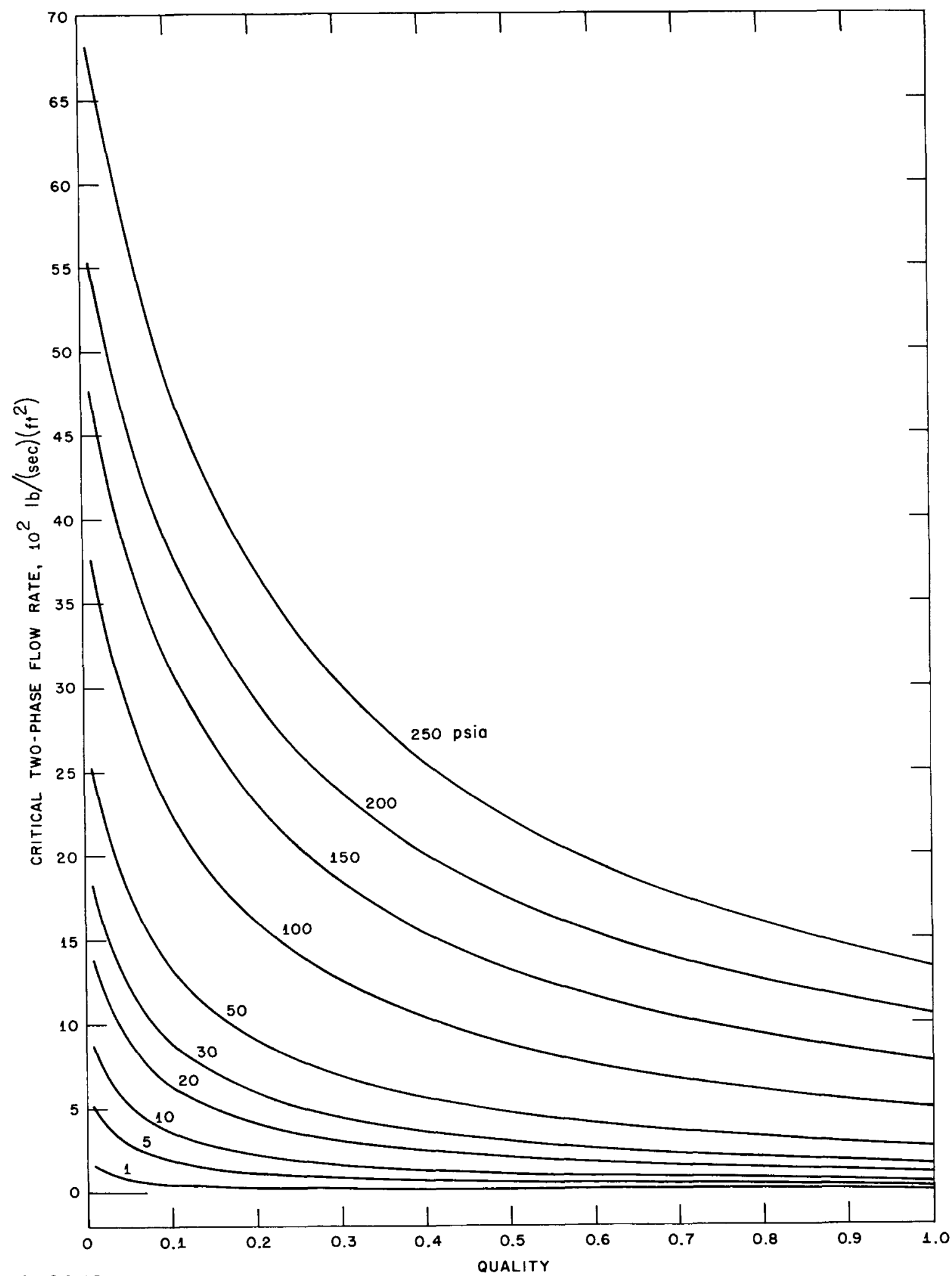

$112-3152$

Fig. 13. Theoretical Prediction of Two-phase Critical-flow Rates for Rubidium 
Figure 14 clearly indicates the large difference obtained in criticalflow rates when using a slip model and the homogeneous-flow model. It is to be expected that large slip ratios exist for liquid-metal systems for the large pressure gradients prevailing under critical-flow conditions. Since the ratio of the densities of the liquid state to the vapor state is usually large for metallic fluids, it is reasonable to conclude that the lighter phase will be accelerated significantly more than the heavier phase when large pressure drops are occurring. As the calculations show, the momentum due to forced convection are smaller for slip than for no-slip flow. Consequently, for a given $\triangle p$, a larger total flow rate is obtained for slip than for no-slip flow

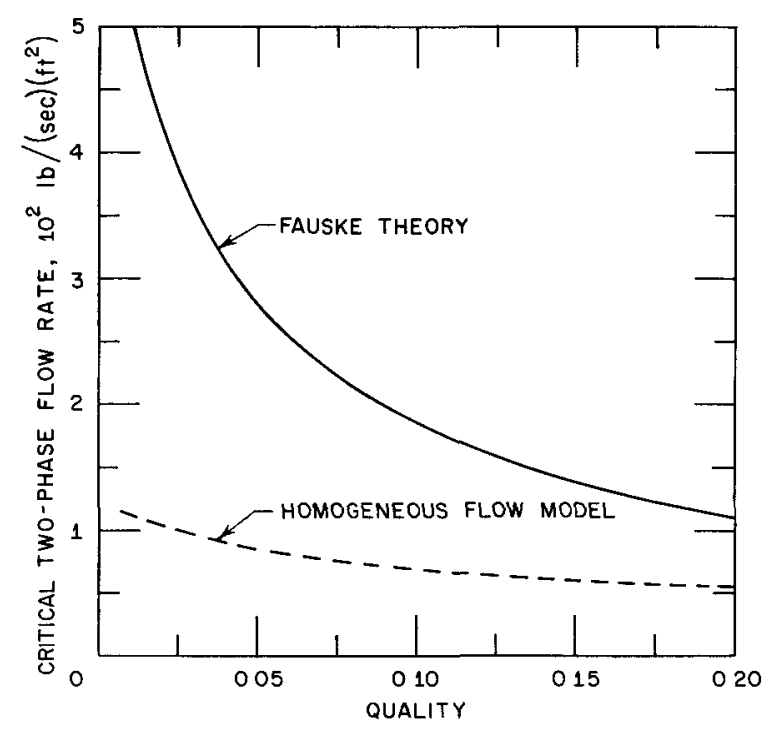

$$
\text { Fig. } 14
$$

Comparison of Flow Rates Calculated from Fauske Theory and Homogeneousequilibrium Theory for Rubidium at 5 psia

As a final remark, although the theory has been proven satisfactorily for steam-water systems, the numerical results given herein must be considered estimates, and experimental verification of the theory for critical flow of liquid metals is needed 
APPENDIX A

FORTRAN LISTING PROGRAM

C FAUSKE THEORY FOR LIQUID METAL SYSTEMS

DIMENSION RECDRD (12), X(20), VG (20), VF (20), SG (20), SF(20), XDVGDP $(20), D S G D P(20), D S F D P(20), P(20), X K(20), D X D P(20), G(20), A(20)$

1 FORMAT (2I 4 )

2 FORMAT (6E12.6)

3 FORMAT (12A6)

4 READ INPUT TAPE $7,3,(\operatorname{RECORD}(\mathrm{J}), \mathrm{J}=1,12$ )

5 READ INPUT TAPE 7,1 , IMAX, JMAX

6 READ INPUT TAPE $7,2,(X(J), J=1, J M A X)$

7 READ INPUT TAPE 7,2 , (VG (I), I $=1$, IMAX)

8 READ INPUT TAPE $7,2,(V F(I), I=1, I M A X)$

10 READ INPUT TAPE $7,2,(S F(I): I=1$, IMAX)

11 READ INPUT TAPE 7,2, (DVGDP(I), I=1, IMAX)

12 READ INPUT TAPE 7,2, (DSGDP (I): I=1; IMAX)

13 READ INPUT TAPE 7,2 , (DSFDP (I): I=1: IMAX)

14 READ INPUT TAPE $7,2,(P(1), I=1, I M A X)$

16 WRITE DUTPUT TAPE $6,3,($ RECORD $(j), j=1,12)$

17 WRITE OUTPUT TAPE 6,51

18 WRITE OUTPUT TAPE $6,52,(P(I), I=1, I M A X)$

19 WRITE DUTPUT TAPE 6,53

20 WRITE DUTPUT TAPE $6: 52,(X(\mathrm{~J}), \mathrm{J}=1, \mathrm{JMAX})$

21 WRITE OUTPUT TAPE $6: 54$

22 WRITE OUTPUT

23 WRITE OUTPUT

25 WRITE OUTPUT

$6,52,(V G(I), I=1, I M A X)$

TAPE 6,55

(VFE (I), I = 1, I MAX)

6.56

TAPE $6,52,(S G(I), I=1,[M A X)$

WR ITE OUTPUT TAPE 6,5\%

WRITE OUTPUT TAPE 6,58

WR ITE OUTPUT TAPE $6,52,(D V G D P(1), I=1, I M A X)$

WRITE DUTPUT TAPE 6,59

4 WRITE DUTPUT TAPE 6,52, (DSFDP(I), I=1, IMAX)

$35 \mathrm{GC}=32 \cdot 2$

36 DO $4 \overline{8} I=1$, IMAX

$L=I-1$

$37 \times K(I)=S Q R T F(V G(I) / V F(I))$

38 DO4 $\mathrm{J}=1, \mathrm{JMAX}$

39 DXDP $(J)=-(1 . /(S G(I)-S F(I)) *((1 .-X(J)) * D S F D P(I)+X(J) * D S G D P(I))$

$40 \mathrm{G}(\mathrm{J})=$ SQRTF $(i-144 * X K(I) * G \mathrm{C}) /((1 ;-X(\mathrm{~J})+X K(I) * X(J)) * X(J) * D V G D P(I)$

$1+(V G(I) *(1 .+2 * x K(I) * x(j)-2 . * x(j) j+V F(I) *(2 * x K(I) * x(j)-2 * * x K(I)$

2-2*XK(I)*XK(I)*X(J)+XK(I)*XK(I)))*DXDP(J))

$41 A(j)=1.1(1 .+(1 . / \times K(1)) *(1 . / \times(j)-1)$.

IF $(L-3 *(i, 3) ; 1000,44,45$

44 WRITE DUTPUT TAPE $6,3,(R E C O R D(K), K=1,12)$

45 WRITE OUTPUT TAPE 6,61, P (I)

46 WRITE OUTPUT TAPE 6,62, XK(I)

72 WRITE OUTPUT TAPE 6.63

73 WRITE DUTPUT TAPE $6,64,(X(J), D X D P(J), A(J),(j(J), J=1, J M A X)$

48 CONTINUE

$49 \mathrm{GO}$ TO 4

1000 WRITE OUTPUT TAPE 6.65

CALL SYSTEM

51 FORMAT ( $40 X, 19 H P R E S S U R E$ INPUT DATA)

52 FORMAT (6E 15.6$)$

53 FORMAT ( $40 X, 18$ HQUALITY INPUT DATA)

54 FORMAT ( $40 X$, I 3 HVG INPUT DATA)

55 FORMAT ( $40 X, 13$ HVF INPUT DATA)

56 FORMAT (40X: I 3HSG INPUT DATA)

57 FORMAT ( $40 X, 13$ HSF INPUT DATA)

58 FORMAT (40X, 16 HDVGDP INPUT DATA)

59 FORMAT $(40 X, 16 H D S G D P$ INPUT DATA)

60 FORMAT $(40 X, 16 H D S F D P$ INPUT DATA

61 FORMAT ( 1 HO, $41 \times, 16$ HPRESSURE, PSIA $=E 12.6$ )

62 FORMAT (IH,41X, 15HSLIP RAIIO K $=E 13.6$ )

63 FORMAT ( IHO, $61 X, 4$ HVOID, $11 X, 4$ HMASS/IH, $28 X$, 9HOUALITY, $X$,

$X 8 X, 5$ HDX /DP, $8 X, 1$ OHFRACTION, A, $5 X, 1$ OHVELOCITY, G)

64 FORMAT (IH:23X,E15,6,1X,E15.6,E15.6,E15.6)

65 FDRMAT ( 1 HO, 5 HERROR)

END 11 , 1,0 ) 
APPENDIX B

COMPUTER OUTPUT PRINT OUTS OF CRITICAL FLOW FOR LIQUID METALS 
Table 1

COMPUTER INPUT DATA FOR RUBIDIUM

\begin{tabular}{|c|c|c|c|c|}
\hline 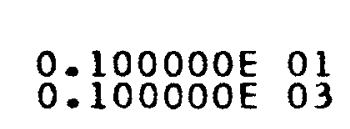 & $\begin{array}{l}0.500000 E \\
0.150000 E\end{array}$ & 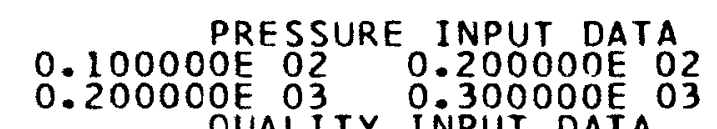 & $0.300000 E 02$ & $0.500000 E \quad 02$ \\
\hline $\begin{array}{l}1.000000 E-02 \\
0.400000 \mathrm{E}-00 \\
0.950000 \mathrm{E} \mathrm{O0}\end{array}$ & $\begin{array}{l}0.300000 E-01 \\
0.500000 E 00\end{array}$ & $\begin{array}{l}0.500000 \mathrm{QULIITY} \\
0.500000 \mathrm{E}-00 \\
0.60000 \mathrm{O}\end{array}$ & $\begin{array}{l}0.200000 E-00 \\
0.800000 E \text { OD }\end{array}$ & $\begin{array}{l}0.300000 E-00 \\
0.900000 E\end{array}$ \\
\hline $\begin{array}{l}0.159000 E \\
0.250000 \mathrm{E}\end{array}$ & $\begin{array}{l}0.380000 E \\
0.172000 E\end{array}$ & $\begin{array}{ll}\text { OOOE } & 02 \\
\text { OOOE OO }\end{array}$ & $0.725000 E 01$ & $0.465000 E 01$ \\
\hline $\begin{array}{l}0.117330 \mathrm{E}-01 \\
0.127760 \mathrm{E}-01\end{array}$ & $\begin{array}{l}0.119850 \mathrm{E}-01 \\
0.129440 \mathrm{E}-01\end{array}$ & $\begin{array}{l}0.121210 \mathrm{E}-01 \\
0.130780 \mathrm{E}-01\end{array}$ & $0.123850 E-01$ & $0.125350 E-01$ \\
\hline $\begin{array}{l}0.584200 E \text { OO } \\
0.504400 \mathrm{E}\end{array}$ & $\begin{array}{l}0.556000 \mathrm{E} O 00 \\
0.498400 \mathrm{E}-00\end{array}$ & $\begin{array}{l}0.543000 \mathrm{O} 00 \\
0.494500 \mathrm{E}-00 \\
.40\end{array}$ & $0.523600 E \quad 00$ & $0.515000 E 00$ \\
\hline $\begin{array}{l}0.315600 \mathrm{E}-00 \\
0.358900 \mathrm{E}-00\end{array}$ & $\begin{array}{l}0.328100 E-00 \\
0.364100 \mathrm{E}-00\end{array}$ & & $0.345000 E-00$ & $0.350500 E-00$ \\
\hline $\begin{array}{l}-0.127500 E-03 \\
-0.240000 E-01\end{array}$ & $\begin{array}{l}-0.750000 E \text { O } 1 \\
-1.000000 \mathrm{E}-02\end{array}$ & 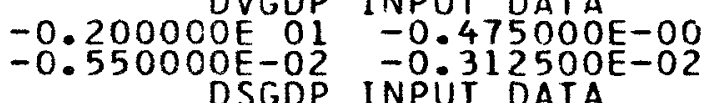 & $-0.225000 E-00$ & $-0.850000 E-01$ \\
\hline $\begin{array}{l}-0.180000 E-01 \\
-0.160000 E-03\end{array}$ & $\begin{array}{l}-0.338000 E-02 \\
-1.00000 E-04\end{array}$ & $\begin{array}{lll}-0.200000 E-02 & -0.800000 E-03 \\
-0.700000 E-04 & -0.350000 E-04 \\
\text { DSFDP }\end{array}$ & $-0.560000 E-03$ & $-0.300000 E-03$ \\
\hline $\begin{array}{l}0.750000 \mathrm{E}-02 \\
0.725000 \mathrm{E}-03\end{array}$ & $\begin{array}{l}0.180000 \mathrm{E}-02 \\
0.900000 \mathrm{E}-04\end{array}$ & $\begin{array}{l}0.900000 \mathrm{E}-03 \\
0.725000 \mathrm{E}-04\end{array}$ & $0.350000 \mathrm{E}-03$ & $0.220000 E-03$ \\
\hline
\end{tabular}


Table 2

CRITICAL FLOW DATA FOR RUBIDIUM

PRESSURE,PSIA $=0.100000 E 01$ SLIP RATIO K $=0.116411 E 03$

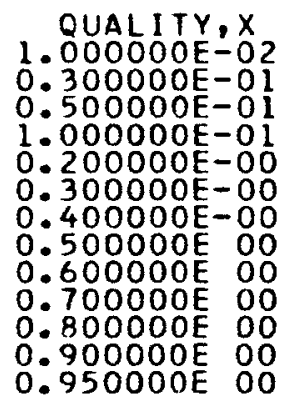

OUALITY,X

1. 00000OE-02

$0.500000 \mathrm{E}-0 \mathrm{I}$

1. $000000 \mathrm{E}-01$

$0.200000 \mathrm{E}-00$

$0.300000 E-00$

$0.400000 \mathrm{E}-0 \mathrm{O}$

0.500000 E 00

$0.600000 E$ OO

$0.700000 E$ DO

0.800000 OO

$0.900000 E$ OO

0.950000 E רO

QUALITY,X

1. 0OOOOOE-02

$0.300000 \mathrm{E}-01$

$0.500000 \mathrm{E}-01$

1.000000E-01

$0.200000 \mathrm{E}-00$

$0.300000 E-00$

$0.400000 E-00$

0.500000 E OO

0.600000 E 00

0.700000 E 00

0.800000 E DO

0.900000 E 00

0.950000 E OD

QUALITY, $X$

1. 000000E-02

0.300000 E- 01

$0.500000 \mathrm{E}-01$

1. $000000 \mathrm{E}-01$

$0.200000 \mathrm{E}-00$

$0.300000 E-00$

0.400000 E- 00

0.500000 E 00

0.600000 E 0

0.700000 E 00

0. B00000E OO

0.900000 E OO

0.950000 E 00
VOID

FRACTI ON, A

$0.540413 \mathrm{E}$ OO

$0.782625 \mathrm{E}$ OD

$0.859686 \mathrm{E} 00$

0.928236 E OO

0.966780 E DO

0.980350 E OO

$0.987279 E$ OO

$0.991483 \mathrm{E}$ OO

$0.994306 \mathrm{E} \mathrm{OO}$

$0.996332 \mathrm{E}$ OO

0.997857 E OO

$0.999046 \mathrm{E}$ OD

$0.999548 E$ OO
PRESSUREPPSIA $=0.500000 E$ OI

$-0.721632 \mathrm{E}-02$

$-0.676174 \mathrm{E}-02$

$-0.335235 \mathrm{E}-02$

$-0.107942 \mathrm{E}-02$

$0.119351 \mathrm{E}-02$

$0.346643 \mathrm{E}-02$

$0.573936 \mathrm{E}-02$

$0.102852 \mathrm{E}-01$

$0.125581 \mathrm{E}-01$

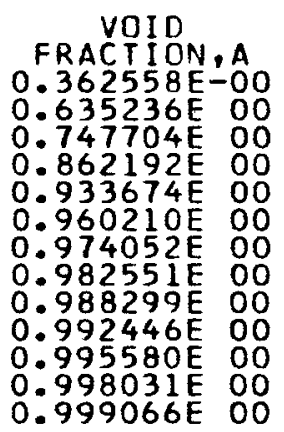

PRESSURE,PSIA $=0.100000 E 02$ SLIP RATIO K $=0.406205 \mathrm{E}$ O2

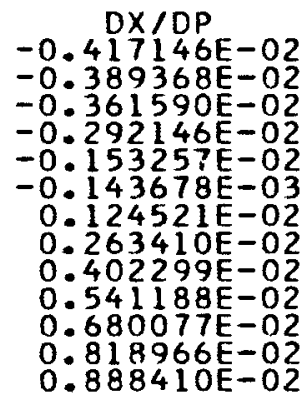

FRACTION, A

$0.290935 \mathrm{E}-00$

0.556798 E DO

$0.681318 E$ OO

$0.818624 \mathrm{E} \quad 00$

$0.910355 \mathrm{E}$ OO

0.945678 E 00

$0.964388 \mathrm{E} \quad 00$

$0.975973 \mathrm{E}$ OD

$0.983853 \mathrm{E} 00$

$0.989560 \mathrm{E}$ OO

$0.993883 \mathrm{E}$ DO

$0.997272 \mathrm{E} \quad 00$

$0.998706 \mathrm{E}$ OO

PRESSURE, PSIA $=0.200000 E 02$ SLIP RATIO KA $=0.295184 \mathrm{E} 02$

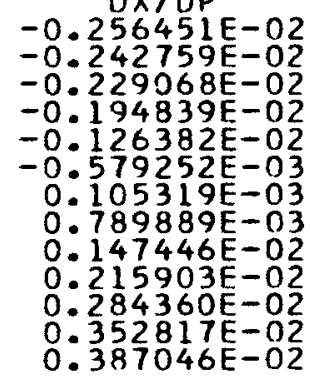

VOID

FRACTI ON, A

$0.229682 \mathrm{E}-00$

$0.477244 E-00$

$0.608396 \mathrm{E}$ OO

$0.766345 \mathrm{E}$ OO

$0.880663 E$ OO

$0.926744 E$ OO

0.951642 E DO

$0.967233 \mathrm{E}$ OO

$0.977914 \mathrm{E}$ OD

0.985689 E 00

$0.991602 \mathrm{E} \mathrm{OD}$

0.996250 E DO

$0.998220 \mathrm{E}$ OO
$-0.767091 \mathrm{E}-02$

$-0.562527 \mathrm{E}-02$
MASS

VELOC ITY, G $0.160077 E^{0} 3$

$0.101548 E \quad 03$

0.763354 E 02

0.481558 E 02

$0.281342 E$ O2

$0.199492 \mathrm{E} 02$

$0.154684 E$ O2

$0.126358 E \quad 02$

0.106817 E 02

0.925188 E 01

$0.816003 E$ OL

$0.729889 \mathrm{E}$ OI

$0.693312 E$ OI

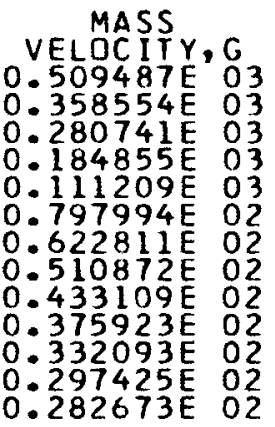

MASS

VFLOC ITY, G

$0.859168 \mathrm{E}^{0} 3$

$0.637263 \mathrm{E} 03$

$0.511944 \mathrm{E} \quad 3$

$0.347696 \mathrm{E}$ O3

$0.214249 \mathrm{E}$ O3

$0.155355 \mathrm{E} \quad 3$

$0.121973 \mathrm{E} 03$

$0.100436 \mathrm{E} 03$

$0.853783 \mathrm{E} 02$

$0.742535 \mathrm{E} \quad 2$

$0.656970 \mathrm{E}$ O2

0.589106 E 02

$0.560179 \mathrm{E}$ O2

MASS

VELOCITY, G

$0.135774 E^{\prime} 04$

$0.106989 \mathrm{E} \quad 04$

$0.890743 \mathrm{E} \quad 03$

$0.636233 E \quad 03$

0.410557 E 03

$0.304507 \mathrm{E} 03$

0.242349 E 03

0.201385 E 03

0.172316 E 03

$0.150605 E$ O3

$0.133764 \mathrm{E} 03$

$0.120318 E$ O3

$0.114562 \mathrm{E} \quad 03$ 
Table 2 (Contd.)

PRESSURE, PSIA $=0.300000 E 02$ SLIP RATIO K $=0.241947 \mathrm{E} 02$

QUALITY, X 1. 000000E-02 $0.300000 E-01$ $0.500000 \mathrm{E}-01$ 1. $000000 E-01$ $0.200000 E-00$ $0.300000 \mathrm{E}-00$ $0.400000 E-00$ 0.500000E 00 0.600000 E 00 0.700000 E 00 0.800000 E 0.900000 E 00 0.950000 E OO
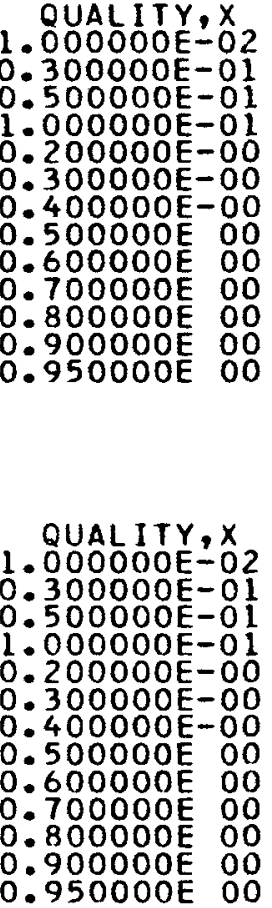

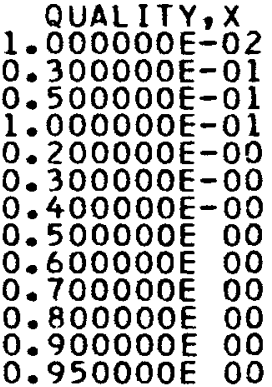

\begin{tabular}{|c|}
\hline 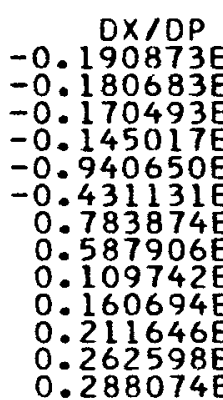 \\
\hline
\end{tabular}

VOID

FRACTION,A

$0.196394 \mathrm{E}-00$

$0.428013 \mathrm{E}-00$

$0.560132 \mathrm{E} 00$

0.728873 E 00

$0.858130 \mathrm{E}$ OO

$0.912043 \mathrm{E} \quad 00$

$0.941622 \mathrm{E}$ OO

$0.960309 \mathrm{E} \mathrm{OO}$

$0.982595 \mathrm{E} \quad 00$

$0.995429 E$ OO

0.997829 E 00 SLIP RATIO K $=0.192604 E 02$

VOID

$-0.0 X / D P$

$-0.117933 \mathrm{E}-02$

$-0.102128 \mathrm{E}-02$

$-0.705167 \mathrm{E}-03$

$-0.389058 \mathrm{E}-03$

$-0.729483 E-04$

$0.243161 \mathrm{E}-03$

$0.559271 \mathrm{E}-03$

$0.875380 \mathrm{E}-03$

$0.119149 \mathrm{E}-02$

$0.150760 \mathrm{E}-02$

FRACTION, A

$0.162864 \mathrm{E}-00$

$0.373308 \mathrm{E}-00$

$0.503402 \mathrm{E}$ OO

$0.681533 \mathrm{E}$ DO

$0.828034 \mathrm{E} 00$

$0.891944 \mathrm{E} \quad 00$

$0.927747 \mathrm{E}$ DO

$0.950643 \mathrm{E}$ OO

$0.966545 \mathrm{E} 00$

$0.978233 \mathrm{E}$ 0O

$0.987186 \mathrm{E} 00$

$0.994264 \mathrm{E} 00$

$0.997275 \mathrm{E} 00$

PRESSURE PSIA $=0.100000 E$ O3

$-0.839519 \mathrm{E}-03$

$-0.800344 \mathrm{E}-03$

$-0.761168 \mathrm{E}-03$

$-0.663230 \mathrm{E}-03$

$-0.467354 \mathrm{E}-03$

$-0.271478 \mathrm{E}-03$

$-0.756014 \mathrm{E}-04$

$0.120275 \mathrm{E}-03$

$0.316151 \mathrm{E}-03$

$0.512027 \mathrm{E}-03$

$0.707904 \mathrm{E}-03$

$0.903780 \mathrm{E}-03$

$0.100172 E-02$

PRE SSURE, PSIA =

$0.150000 \mathrm{E} \quad 03$

$0.301986 \mathrm{E}-00$

$0.424043 \mathrm{E}-00$

$0.608501 \mathrm{IE}$ OO

$0.777636 \mathrm{E} 00$

$0.857043 \mathrm{E} \quad 00$

$0.903154 \mathrm{E}$ 00

$0.933282 \mathrm{E} \quad 00$

$0.954510 \mathrm{E}$ OO

$0.970273 \mathrm{~F} 00$

$0.982442 \mathrm{E}$ DO

0.992120 E DO

$0.996252 \mathrm{E} \quad 00$
$0.973185 \mathrm{E}$ 00

0.989773 E 00

PRESSURE, PSIA $=0.500000 E 02$

$-0.130578 \mathrm{E}-02$

$0.123805 \mathrm{E}-0$

\section{SLIP RATIOK $=0.115274 \mathrm{E} 02$}

$\mathrm{D} \times / \mathrm{DP}$

$-0.655994 \mathrm{E}-03$

$-0.627699 \mathrm{E}-03$

$-0.599404 \mathrm{E}-03$

$-0.528667 \mathrm{E}-03$

$-0.387193 E-03$

$-0.245719 \mathrm{E}-03$

$-0.104244 \mathrm{E}-03$

$0.372301 \mathrm{E}-04$

$0.178704 \mathrm{E}-03$

$0.320179 \mathrm{E}-03$

$0.461653 \mathrm{E}-03$

$0.673864 \mathrm{E}-03$
VOID

FRACTION, A

$0.104294 \mathrm{E}-00$

$0.262818 \mathrm{E}-00$

$0.377608 E-00$

0.742390 E 00

$0.831658 \mathrm{E} \quad 00$

$0.884858 E$ OO

$0.920175 E$ OO

$0.945328 E \quad 00$

$0.964154 \mathrm{E} \quad 00$

0.978773 E OO

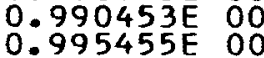

$0.603127 \mathrm{E}-03$

$0.561561 \mathrm{E}$ 00
MASS

VELOCITY, G

$0.144083 \mathrm{E} 04$

0.121912 E 04

$0.890088 \mathrm{E} 03$

$0.585293 \mathrm{E} 03$

$0.437959 \mathrm{E} \quad 3$

$0.350382 \mathrm{E} \quad 03$

0.292166 E 03

$0.250612 \mathrm{E} 03$

$0.219442 \mathrm{E} 03$

$0.195186 \mathrm{E} \quad 3$

$0.175769 \mathrm{E} 03$

$0.167444 \mathrm{E}$ O3

MASS

VELOCITY, G

$0.247147 \mathrm{E}^{04}$

$0.206692 \mathrm{E} \quad 04$

0.178520 E 04

$0.134361 \mathrm{E} 04$

$0.909306 \mathrm{E} \quad 03$

$0.690304 E \quad 03$

0.557166 E 03

$0.467389 \mathrm{E} \quad 03$

0.402662 E 03

$0.353747 \mathrm{E} 03$

$0.315465 E$ O3

0.284679 E 03

$0.271440 \mathrm{E} 03$
MASS

VELOC ITY, G $0.372484 \mathrm{E}^{04}$ 0.324387 E 04 0.288221 E 04 $0.226927 \mathrm{E} \quad 04$ $0.160833 E 04$ 0.125128 E 04 $0.102572 \mathrm{E} \quad 04$ $0.869735 \mathrm{E} 03$ $0.755243 \mathrm{E} 03$ 0.667546 E 03 $0.598184 \mathrm{E} 03$ $0.541931 \mathrm{E} 03$ $0.517607 E \quad 03$ 
Table 2 (Contd.)

PRESSURE PSIA $=0.200000 E 03$
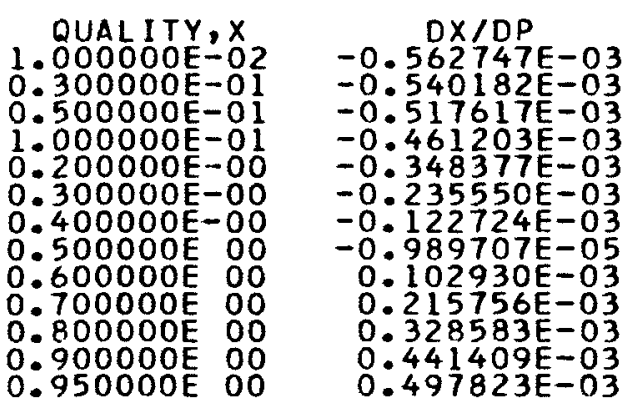

VOID

FRACTIDN, A

$0.930749 E-01$

$0.348424 \mathrm{E}-00$

$0.530273 \mathrm{E} 00$

0.717515 E DO

$0.813235 \mathrm{E} 00$

$0.871356 \mathrm{E} \quad 00$

$0.910395 \mathrm{E}$ OO

$0.938424 \mathrm{E}$ DO

$0.959525 \mathrm{E} 00$

$0.975985 E$ OD

$0.989182 \mathrm{E} \quad 00$

$0.994846 \mathrm{E} \quad 00$

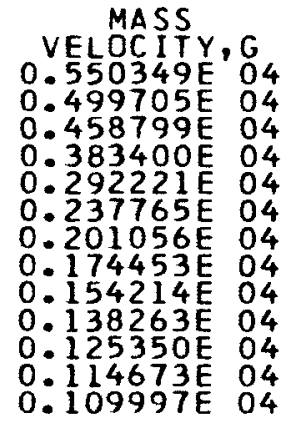

PRESSURE, PSIA =

SLIP RATIO K =

$\begin{array}{ll}0.300000 E & 03 \\ 0.858944 E & 01\end{array}$

QUAL ITY:X

1.000000E-02

$0.300000 \mathrm{E}-01$

$0.500000 \mathrm{E}-01$

1.000000E-01

$0.200000 E-00$

$0.300000 \mathrm{E}-00$

$0.400000 \mathrm{E}-00$

0.500000 E 00

0.600000 E 00

0.700000 E 00

0.800000 E 00

0.900000 E 0

VOID

FRACTION, A

DX/DP
$-0.448134 \mathrm{E}-03$

$-0.432943 E-03$

$-0.417752 \mathrm{E}-03$

$-0.379774 \mathrm{E}-03$

$-0.303819 E-03$

$-0.227865 \mathrm{E}-03$

$-0.151910 \mathrm{E}-03$

$-0.759549 E-04$

$-0.592119 \mathrm{E}-11$

$0.759549 E-04$

$0.151910 E-03$

$0.227865 \mathrm{E}-03$

$0.798354 \mathrm{E}-01$

$0.209894 E-00$

$0.311331 \mathrm{E}-00$

$0.488329 E-00$

$0.682273 \mathrm{~F} 00$

$0.786379 E$ OO

0.851330 E OO

$0.895719 \mathrm{~F}$ OO

$0.927975 \mathrm{E}$ OO

$0.952476 E$ OO

0.971718 E 00

$0: 987229 E$ OO

0.109997 E 04

$0.950000 E$ DO

0.993910 E DO

MASS

VELOCITY, G $0.620154 \mathrm{E} 04$ $0.572880 \mathrm{E} 04$ $0.483098 E \quad 04$ $0.370686 \mathrm{E} \quad 04$ 0.302060 E 04 $0.255386 \mathrm{E} 04$ $0.221436 E \quad 04$ 0.195570 E 04 $0.175178 E \quad 04$ 0.158675 E 04 $0.145037 \mathrm{E} \quad 04$ 
Table 3

COMPUTER INPUT DATA FOR LITHIUM

\begin{tabular}{|c|c|c|c|c|}
\hline $\begin{array}{l}0.200000 E \\
0.100000 \mathrm{E}\end{array} 0 \frac{1}{3}$ & $0.500000 E 01$ & 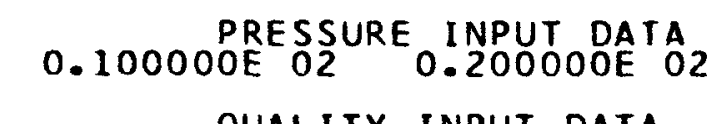 & $0.300000 E 02$ & $0.500000 E \quad 02$ \\
\hline $\begin{array}{l}1.000000 E-02 \\
0: 400000 E-00 \\
0.950000 E 00\end{array}$ & $\begin{array}{l}0.300000 E-01 \\
0.500000 E \text { OD }\end{array}$ & 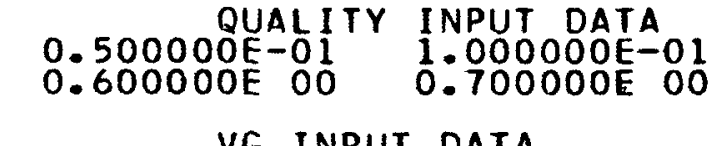 & $\begin{array}{l}0.200000 \mathrm{E}-00 \\
0.800000 \mathrm{E} 00\end{array}$ & $\begin{array}{l}0.300000 E-00 \\
0.900000 E \quad 00\end{array}$ \\
\hline $\begin{array}{l}0.180000 E \\
0.460000 E\end{array}$ & $0.740000 E \quad 03$ & $0.390000 E$ O3 O. O.205000E 03 & $0.138500 E \quad 03$ & $0.870000 \mathrm{E} 02$ \\
\hline $\begin{array}{l}0.367900 E-01 \\
0.401400 E-01\end{array}$ & $0.373500 E-01$ & $010.384150 E-01$ & $0.387800 E-01$ & $0.342750 E-01$ \\
\hline $\begin{array}{l}0.624700 E \\
0.531100 E \text { O1 } \\
0.51\end{array}$ & $0.600100 E 01$ & & $0.557400 E 01$ & $0.546700 \mathrm{E} 01$ \\
\hline $\begin{array}{l}0.268680 E \text { O } \\
0.302900 \mathrm{E} \\
01\end{array}$ & $0.276280 E \quad 01$ & $0.281880 \mathrm{E} \mathrm{OI} \quad 0.287730 \mathrm{E} \mathrm{Ol}$ & $0.291440 E 01$ & $0.296250 E$ \\
\hline $\begin{array}{l}-0.850000 \mathrm{E} \quad 03 \\
-0.450000 \mathrm{E}-00\end{array}$ & $-0.137500 E \quad 03$ & $\begin{array}{c}-0.400000 E \text { O2 } \\
\text { DSGDP INPUT DATA }\end{array}$ & $-0.425000 \mathrm{E} \mathrm{O1}$ & $-0.800000 E 00$ \\
\hline $\begin{array}{l}-0.120000 E-00 \\
-0.200000 E-02\end{array}$ & $-0.500000 E-01$ & $\begin{array}{r}-0.250000 \\
0\end{array}$ & $-0.800000 E-02$ & $-0.460000 E-02$ \\
\hline $\begin{array}{l}0.3200 \\
0.1025\end{array}$ & $.165000 \mathrm{E}$ & & $0.300000 E-02$ & 0.180000 \\
\hline
\end{tabular}


Table 4

CRITICAL FLOW DATA FOR LITHIUM

PRESSURE,PSIA $=0.200000 E$ O01
SLIP RATIOK $=0.221193$ E 03

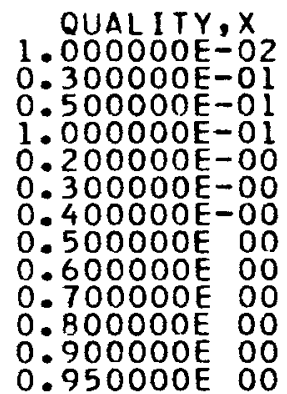

QUAL I TY,X

1.000000E-02

$0.300000 \mathrm{E}-01$

$0.500000 \mathrm{E}-01$

1.000000E-O 1

$0.200000 \mathrm{E}-00$

$0.300000 E-00$

$0.400000 \mathrm{E}-00$

$0.500000 \mathrm{E}$ OO

$0.600000 E$ OO

0.700000 E 00

0.800000 E 00

0.900000 E 00

0.950000 E 00

QUALITY, X 1. 00000OE-02 $0.300000 E-01$ $0.500000 \mathrm{E}-01$

1.000000E-01

$0.200000 \mathrm{E}-00$

0.300000 E- 00

$0.400000 E-00$

0.500000 E 00

0.600000 E OO

0.700000 E 00

$0.800000 \mathrm{E}$ OO

0.900000 E 00

0.950000 E 00

QUAL ITY, $X$

1. 0000OOE-02

$0.300000 \mathrm{E}-01$

$0.500000 \mathrm{E}-01$

$1.000000 \mathrm{E}-01$

$0.200000 \mathrm{E}-00$

$0.300000 \mathrm{E}-00$

$0.400000 \mathrm{E}-00$

0.500000 E 00

0.600000 E 00

0.700000 E 00

0.800000 E 00

$0.950000 E$ OO
VOID

FRACTIDN, A

$-0.856132 \mathrm{E}-02$

$-0.770743 \mathrm{E}-02$
$-0.685355 \mathrm{~F}-02$

$-0.471884 \mathrm{E}-02$

$-0.449413 \mathrm{E}-03$

$0.382001 \mathrm{E}-02$

$0.123589 \mathrm{E}-01$

$0.166283 \mathrm{E}-01$

$0.208977 \mathrm{E}-01$

$0.251671 \mathrm{E}-01$

$0.294365 \mathrm{E}-01$

$0.690811 \mathrm{E}$ OO

$0.872466 \mathrm{E} \quad 00$

$0.920897 \mathrm{E}$ 0O

$0.960902 E$ OO

0.982237 E 00

$0.989561 \mathrm{E} 00$

$0.993264 \mathrm{E} \quad 00$

$0.995499 E$ OO

$0.996995 \mathrm{E}$ OD

$0.998066 \mathrm{E} \mathrm{OO}$

$0.998871 \mathrm{E}$ OO

$0.999498 \mathrm{E}$ OO

PRESSURE,PSIA $=0.500000 E 01$

SLIP RATIO K $=0.140757 \mathrm{~K} 03$

VOID

$D X / D P$

$-0.489006 E-02$

$-0.447934 \mathrm{E}-02$

$-0.406862 \mathrm{E}-02$

$-0.304181 \mathrm{E}-02$

$-0.988203 \mathrm{E}-03$

$0.106541 \mathrm{E}-02$

$0.311902 \mathrm{E}-02$

$0.517263 \mathrm{E}-02$

$0.927985 \mathrm{E}-02$

$0.113335 \mathrm{E}-01$

$0.133871 \mathrm{E}-01$

$0.144139 \mathrm{E}-01$

PRESSURE, PSIA $=0.100000 E 02$ SLIP RATIO K $=0.101510 \mathrm{E} 03$

FRACTION, A

$0.587082 E$ OO

$0.881070 \mathrm{E}$ OO

$0.939903 \mathrm{E} \quad 00$

$0.972368 \mathrm{E}$ OO

$0.983693 \mathrm{E}$ OO

$0.989456 \mathrm{E} 00$

$0.992946 \mathrm{E}$ OO

$0.995286 \mathrm{E} 00$

$0.996964 \mathrm{~F} 00$

$0.998227 \mathrm{E} 00$

$0.999211 \mathrm{E} 00$

$0.999626 \mathrm{E} \quad 00$

\section{VOID}

$D \times 1 O P$
$-0.244528 \mathrm{E}-02$
$-0.222838 \mathrm{E}-02$
$-0.201148 \mathrm{E}-02$
$-0.146922 \mathrm{E}-02$
$-0.384717 \mathrm{E}-03$
$0.699788 \mathrm{E}-03$
$0.178429 \mathrm{E}-02$
$0.286880 \mathrm{E}-02$
$0.395330 \mathrm{E}-02$
$0.503781 \mathrm{E}-02$
$0.612231 \mathrm{E}-02$
$0.720682 \mathrm{E}-02$
$0.774907 \mathrm{E}-02$

FRACTION, A

$0.506260 \mathrm{E}^{\prime} 00$

$0.758425 \mathrm{E}$ OO

0.842337 E 00

0.918560 E 00

$0.962089 E$ D 00

$0.977530 \mathrm{E}$ OO

$0.985438 E$ OO

$0.990245 \mathrm{E} \quad 00$

$0.993475 \mathrm{E}$ 0O

$0.995796 \mathrm{E}$ DO

$0.997543 \mathrm{E}$ OO

0.998907 E 00

$0.999482 E \quad 00$

PRESSURE,PSIA $=0.200000 E 02$ SLIP RATIO K $=0.730511 \mathrm{E} \mathrm{O2}$

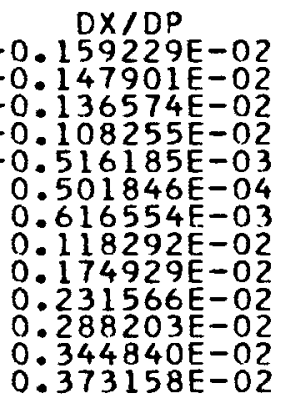

VOID

FRACTIDN, A

$0.424589 \mathrm{E}-00$

$0.693187 \mathrm{E} 00$

$0.793593 E$ OO

$0.890312 \mathrm{E} 00$

$0.948086 \mathrm{E} \quad 00$

$0.969048 E \quad 00$

$0.979880 E$ OO

$0.986496 \mathrm{E}$ OO

0.990957 E 00

0.994167 F 00

0.996589 E 00

$0.998481 \mathrm{E}$
0.90
MASS

VELDC I Y Y, G

$0.904168 \mathrm{E} 02$

$0.503835 \mathrm{E} 02$

$0.356857 \mathrm{E} 02$

$0.209092 \mathrm{E} 02$

$0.115194 \mathrm{E} \quad 2$

$0.796127 \mathrm{E}$ Ol

$0.608450 \mathrm{E} \mathrm{O1}$

$0.492434 \mathrm{E} \mathrm{OI}$

$0.413596 \mathrm{E}$ DI

$0.356527 \mathrm{E}$ OI

$0.313302 \mathrm{E} \mathrm{O1}$

$0.279427 \mathrm{E}$ Ol

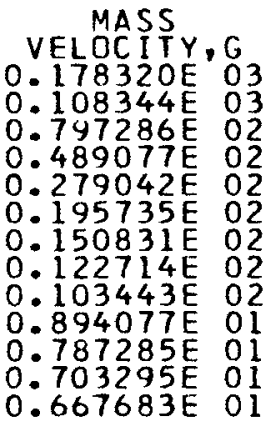

MASS

VELOCITY, G

$0.514442 \mathrm{E} 03$

$0.345392 \mathrm{E} \quad 3$

0.264457 E 03

$0.169448 \mathrm{E} \quad 3$

$0.997576 \mathrm{E} \quad 2$

$0.708981 E$ O2

$0.550314 \mathrm{E} 02$

$0.449804 E 02$

D.380387E 02

$0.329553 \mathrm{E} 02$

0.290715 E 02

$0.260072 \mathrm{E} 02$ 
Table 4 (Contd.)

PRESSURE,PSIA $=0.300000 E$ O2
SLIP RATIO K $=0.597614 \mathrm{E} 2$

QUALITY,X

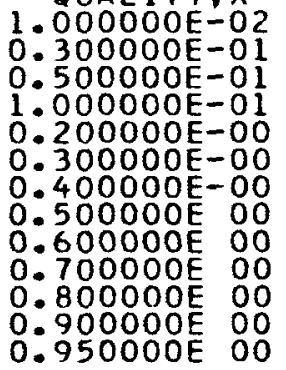

QUALITY, $X$
1. OOOOOOE-O2

1. 0000OOE-02

$0.500000 \mathrm{E}-01$

1. OOOOOOE-01

$0.200000 \mathrm{E}-00$

$0.300000 E-00$

$0.400000 E-00$

$0.500000 E$ OO

$0.600000 E$ OO

0.700000 E 00

0.800000 E OO

$0.900000 E$ OO

$0.950000 E$ OO

QUAL I TY, X

1. OOOOOOE-02

$0.300000 \mathrm{E}-01$

$0.500000 E-01$

1. $000000 \mathrm{E}-01$

$0.200000 E-00$

$0.300000 E-00$

$0.400000 E-00$

0.500000 E 0

0.600000 E DO

0.700000 E 00

0.800000 E 0

$0.900000 E$ OO

FRACTION , A

$D \times / D P$

$-0.108663 E-02$

$-0.100391 \mathrm{E}-02$

$-0.921191 \mathrm{E}-03$

$-0.714393 E-03$

$-0.300797 \mathrm{E}-03$

$0.112799 \mathrm{E}-03$

$0.526395 \mathrm{E}-03$

$0.939991 \mathrm{E}-03$

$0.135359 \mathrm{E}-02$

$0.176718 \mathrm{E}-02$

$0.218078 \mathrm{E}-02$

$0.259438 \mathrm{E}-02$

$0.280117 \mathrm{E}-02$

$0.376423 E-00$

$0.648912 \mathrm{E} \quad 00$

$0.758765 \mathrm{E} \quad 00$

$0.869113 \mathrm{E} \quad 00$

$0.937266 \mathrm{E} 00$

$0.962423 \mathrm{E}$ OO

$0.975515 \mathrm{E} \quad 00$

$0.983542 \mathrm{E}$ O०

$0.988968 \mathrm{E} \quad 00$

$0.992880 \mathrm{E}$ OO

$0.995834 \mathrm{E} \quad 00$

$0.998144 \mathrm{E}$ OO

0.999120 E 00

PRESSURE PSIA $=0.500000 E$ O2
SLIP RATIO K $=0.503815$ E

$\mathrm{DX} / \mathrm{DP}$

$-0.693152 \mathrm{E}-03$

$-0.642044 \mathrm{E}-03$

$-0.590936 \mathrm{E}-03$

$-0.463166 \mathrm{E}-03$

$-0.207626 \mathrm{E}-03$

$0.479137 \mathrm{E}-04$

$0.303454 \mathrm{E}-03$

$0.558994 \mathrm{E}-03$

$0.814534 E-03$

$0.107007 \mathrm{E}-02$

$0.132561 \mathrm{E}-02$

$0.158115 E-02$

$0.170892 \mathrm{E}-02$

PRESSURE, PSIA = SLIP RATIO K =

$D \times / D P$

$-0.435911 E-03$

$-0.409400 E-03$

$-0.382888 \mathrm{E}-03$

$-0.316608 \mathrm{E}-03$

$-0.184049 E-03$

$-0.514399 \mathrm{E}-04$

$0.810692 \mathrm{E}-04$

$0.213628 E-03$

$0.346188 E-03$

$0.478747 \mathrm{E}-03$

$0.611306 E-03$

$0.743865 \mathrm{E}-03$
VOID

FRACTION, A

$0.337267 \mathrm{E}-00$

0.609099 E 00

$0.726152 \mathrm{E} \quad 00$

$0.848438 \mathrm{E} \quad 00$

$0.926446 \mathrm{E}$ DO

$0.955737 E$ OO

$0.971088 \mathrm{E}$ OO

0.980538 E 00

$0.986940 \mathrm{E}$ OO

0.991565 E 00

0.995062 E OO

$0.997799 \mathrm{E} \quad 00$

0.998956 E 00

$0.100000 E 03$ 0.338525 E 02

VOID

FRACI ION, A

0.254812 E-0O

$0.511476 \mathrm{E} \quad 00$

$0.640509 E$ OO

$0.789977 \mathrm{~F} \quad 00$

0.894327 E 00

$0.935518 E$ OO

0.957570 E 00

0.971308 E 00

$0.980687 \mathrm{E}$ OD

0.987498 E 00

$0.992669 \mathrm{E}$ DO

$0.996729 \mathrm{E}$ OO
MASS

VELOCI TY, G

$0.500158 \mathrm{E} \quad 03$

0.389673 E 03

$0.255035 \mathrm{E} 03$

0.152699 E 03

$0.109338 \mathrm{E} 03$

0.852322 E 02

$0.698584 \mathrm{E} \quad 22$

$0.591921 E$ O2

$0.513556 \mathrm{E} 02$

$0.453536 \mathrm{E}$ O2

$0.406088 \mathrm{E} 02$

$0.385904 E \quad 02$

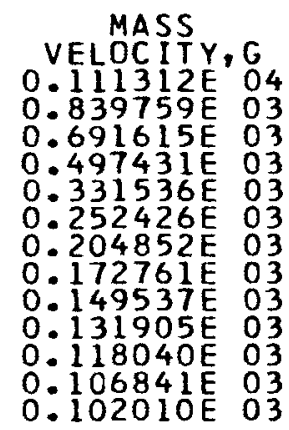

MASS

VELOCITY, G $0.164960 E^{\circ} 04$ D. 125675 E 04 0.102365 E 04

0.707067 E 03

$0.441373 E \quad 03$

$0.321837 \mathrm{E} 03$

$0.253478 E$ E

$0.209144 \mathrm{E} \quad 03$

$0.178040 E$ O3

$0.155003 E$ O3

0.137251 E 03

0.123152 E 03 
Table 5

COMPUTER INPUT DATA FOR MERCURY

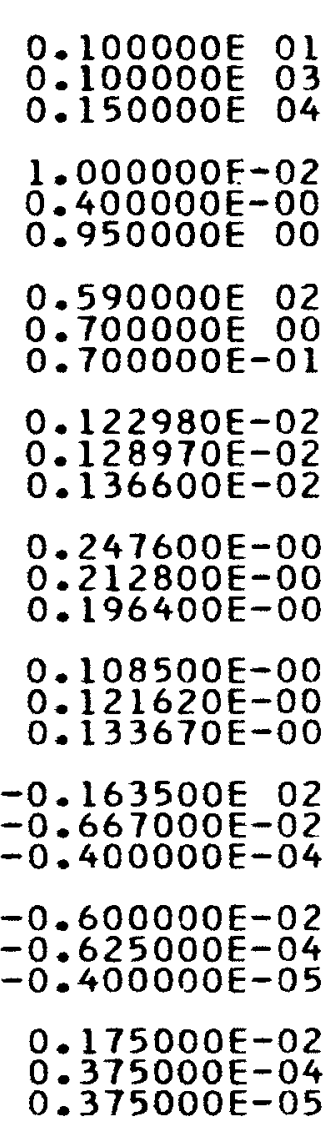

$\begin{array}{ll}0.500000 E & 01 \\ 0.200000 E & 03 \\ 0.200000 E & 04\end{array}$

$0.300000 E-01$
0.500000 E 00

$0.112000 E \quad 02$

$0.390000 \mathrm{E}-00$

$0.540000 \mathrm{E}-01$

$0.124640 \mathrm{E}-02$

$0.130400 \mathrm{E}-02$

$0.137830 \mathrm{E}-02$

$0.233700 E-00$ $0.208300 \mathrm{E}-00$ $0.194900 \mathrm{E}-00$

$0.112890 E-00$ $0.124240 \mathrm{E}-00$ $0.135380 \mathrm{E}-00$

- $0.160000 E$ O1 $-0.150000 \mathrm{E}-02$ $-0.250000 E-04$

$-0.187500 E-02$ $-0.325000 \mathrm{E}-04$
$-0.250000 \mathrm{E}-05$

$0.625000 \mathrm{E}-03$ $0.200000 E-04$ $0.312500 \mathrm{E}-05$
PRESSURE INPUT DATA $\begin{array}{ll}0.100000 E \text { O2 } & 0.200000 E \text { O2 } \\ 0.300000 E \text { 03 } & 0.500000 \mathrm{E}\end{array}$ QUALITY INPUT DATA $\begin{array}{ll}0.500000 E-01 & 1.000000 E-01 \\ 0.600000 E \text { OO } & 0.700000 \text { OD }\end{array}$ VG INPUT DATA

$0.590000 E$ OI O.3000OOE 01 $0.279000 E-00 \quad 0.179000 E-00$ $\begin{array}{ll}\text { VF INPUT DATA } \\ 0.125370 \mathrm{E}-02 & 0.126170 \mathrm{E}-02 \\ 0.131380 \mathrm{E}-02 & 0.132800 \mathrm{E}-02\end{array}$ $0.131380 \mathrm{E}-02$ 0.132800E-02

SG INPUT DATA

$0.229400 E-00$ O.224300E-0O $0.205700 E-00 \quad 0.202400$ E-OO

SF INPUT DATA

$0.114540 \mathrm{E}-00$ 0.116410E-00 $0.125870 \mathrm{E}-00$ 0.128100 E-O0 DVGDP
$-0.550000 \mathrm{~F}$ OD INPUT DATA $\begin{array}{ll}-0.8000 \mathrm{COE}-03 & -0.160000 \mathrm{E}-00 \\ -0.320000 \mathrm{E}-03\end{array}$ DSGDP INPUT DATA

$-0.750000 E-03-0.375000 E-03$ $\begin{array}{ll}-0.750000 E-03 & -0.375000 E-03 \\ -0.212500 E-04 & -0.125000 E-04\end{array}$ DSFDP
$0.275000 E-03$
INPUT DATA
0.150000 $0.275000 \mathrm{E}-03$ $0.137500 E-04$ $\begin{array}{ll}0.300000 E & 02 \\ 0.700000 E & 03\end{array}$

$0.200000 \mathrm{E}-00$

0.200000 E-0O
0.800000 E 00

$0.220000 E^{01}$

$0.133000 \mathrm{E}-00$

$0.126720 \mathrm{E}-02$

$\mathrm{OE}-02$

$0.221300 E-00$
$0.200400 E-00$

$0.117700 E-00$

$0.129690 E-00$

$-0.400000 E-01$
$-0.170000 E-03$

$-0.250000 E-03$

$-0.875000 \mathrm{E}-05$

$0.108000 E-03$

$0.725000 E-05$ $\begin{array}{ll}0.500000 E & 02 \\ 0.100000 E & 04\end{array}$

$0.300000 E-00$ 0.900000 E 00

$0.150000 E$ OL

$0.127740 E-02$
$0.135100 \mathrm{E}-02$

$0.217700 E-00$
$0.198500 \mathrm{E}-00$

$0.119310 E-00$

$0.131500 \mathrm{E}-00$

$-0.300000 E-01$ $-0.900000 E-04$

$-0.137500 E-03$ $-0.562500 E-05$

$0.667000 E-04$ $0.531000 E-05$ 
Table 6

CRITICAL ELOW DATA FOR MERCURY

PRESSUREPPSIA $=0.100000 E$ OL

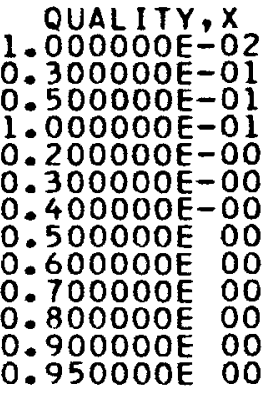

QUALITY, $X$ 1.000000E-02 $0: 300000 \mathrm{E}-01$ $1.000000 \mathrm{E}-01$ $0.200000 \mathrm{E}-00$ $0.300000 \mathrm{E}-00$ $0.400000 E-00$ 0.500000 E 00 $0.600000 \mathrm{E}$ OO 0.700000 E 00 $0.800000 E$ OO $0.900000 E$ OO $0.950000 E$ OO

QUALITY,X 1. 000000E-02 $0.500000 E-01$ 1.000000E-01 $0.200000 E-00$ $0.300000 E-00$ $0.400000 \mathrm{E}-00$ 0.500000 E 00 0.600000 E 00 0.700000 E 00 0.800000 E OO $0.900000 E$
$0.950000 E$

QUAL ITY,X

1. $000000 E-02$

$0.300000 \mathrm{E}-01$

1.000000E-01

$0.200000 \mathrm{E}-00$

$0.300000 \mathrm{E}-00$

$0.400000 E-00$

$0.500000 \mathrm{E} 00$

0.600000 E OO

$0.700000 E$ OO

$0.800000 E$ OD

0.900000 E 00

$0.950000 E$ DO
DX $1 D P$

$-0.120237 \mathrm{E}-01$

- $0.109094 \mathrm{E}-01$

$-0.700935 \mathrm{E}-02$

$-0.143781 \mathrm{E}-02$

$0.413372 \mathrm{E}-02$

$0.970525 \mathrm{E}-02$

$0.152768 \mathrm{E}-01$

$0.208483 \mathrm{E}-01$

$0.264198 \mathrm{E}-01$

$0.319914 \mathrm{E}-01$

$0.375629 \mathrm{E}-01$

$0.403487 \mathrm{E}-01$

PESSURE PSIA

SLIPRATIO $K=$

$0.500000 E$ O 1

FRACTIDN, A

$0.688711 \mathrm{E}$ OO

$0.871369 \mathrm{E}$ O0

$0.960532 \mathrm{E}$ OO

$0.982065 \mathrm{E}$ OO

$0.989459 E$ OO

$0.993198 \mathrm{E} \quad 00$

$0.995455 \mathrm{E}$ 00

$0.996966 \mathrm{E} 00$

$0.998047 \mathrm{E} 00$

$0.998860 \mathrm{E}$ OO

$0.999493 E \quad 00$

$0.999760 \mathrm{E} 00$

$0.947939 \mathrm{E}$ O2

$D \times 10 P$

$-0.496648 \mathrm{E}-02$

$-0.455260 \mathrm{E}-02$

$-0.413873 \mathrm{E}-02$

$-0.310405 \mathrm{E}-02$

$-0.103468 \mathrm{E}-02$

$0.103468 \mathrm{E}-02$

$0.310405 E-02$

$0.517341 \mathrm{E}-02$

$0.931214 \mathrm{E}-02$

$0.113815 \mathrm{E}-01$

$0.144856 \mathrm{E}-01$

PRESSURE, PSIA =

SLIP RATIO K =

FRACIION, A

$0.489148 \mathrm{E}-00$

$0.745662 \mathrm{E} \quad 00$

$0.833031 \mathrm{E} 00$

$0.913290 \mathrm{E} 00$

$0.959512 \mathrm{E} 00$

0.975977 E 00

$0.984423 E$ DO

$0.989561 \mathrm{E} 0 \mathrm{O}$

$0.993016 \mathrm{E}$ OD

$0.995499 E$ OO

0.997370 E 00

$0.998829 E$ OD

$0.999445 \mathrm{E} \quad 00$

$0.100000 \mathrm{E} \quad 02$
$0.724278 \mathrm{E}-02$

$0.134509 \mathrm{E}-01$
MASS

VELOC ITY, G

$0.450168 \mathrm{E}^{03}$

$0.275693 \mathrm{E}$ O3

O.208230E 03

0.134600 E 03

$0.813683 \mathrm{E}$ O2

$0.588252 \mathrm{E} \quad 02$

$0.461743 \mathrm{E}$ O2

$0.380369 \mathrm{E}$ D2

0.323520 E 02

0.281520 E 02

0.249205 E 02

0.223563 E 02

0.212629 E 02

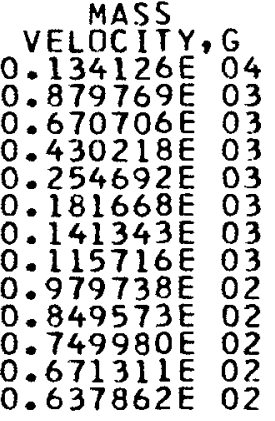

\section{VOID}

FRACTION, A

$-0.230498 \mathrm{E}-02$

$-0.212650 \mathrm{E}-02$

$-0.194802 \mathrm{E}-02$

$-0.150183 \mathrm{E}-02$

$-0.609438 \mathrm{E}-03$

$0.282953 \mathrm{E}-03$

$0.117534 \mathrm{E}-02$

$0.206773 \mathrm{E}-02$

$0.296013 \mathrm{E}-02$

$0.385252 \mathrm{E}-02$

$0.474491 \mathrm{E}-02$

$0.563730 \mathrm{E}-02$

$0.409311 \mathrm{E}-00$

$0.783107 \mathrm{E} 00$

$0.884022 \mathrm{E} D O$

$0.944904 \mathrm{E} \quad 00$

$0.967106 \mathrm{E}$ OD

$0.978602 \mathrm{E} 00$

$0.985632 \mathrm{E} \quad 00$

0.990375 E DO

$0.993791 \mathrm{E}$ OO

$0.996369 \mathrm{E}$ OO

$0.998383 \mathrm{E}$ DO

$0.200000 \mathrm{E} 02$

PRESSURE, PSIA = SLIP RATIO $K=$

$0.200000 E$ O2
$0.487621 \mathrm{E}$

VOID

FRACTION, A

$-0.134164 E-02$

$-0.124432 \mathrm{E}-02$

$-0.114700 \mathrm{E}-02$

$-0.903698 \mathrm{E}-03$

$-0.417091 \mathrm{E}-03$

$0.695152 E-04$

$0.556122 \mathrm{E}-03$

$0.104273 \mathrm{E}-02$

$0.152934 E-02$

$0.201594 \mathrm{E}-02$

$0.250255 E-02$

$0.298916 \mathrm{E}-02$

$0.323246 E-02$
$0.330004 \mathrm{E}-00$

$0.601293 \mathrm{E}$ OO

0.719607 E 00

0.844189 E OO

$0.924188 \mathrm{E}$ OO

$0.954334 E \quad 00$

$0.970156 \mathrm{E} 00$

$0.979904 E$ OO

$0.986513 E$ OO

$0.991288 E$ OO

$0.994899 \mathrm{E}$ OO

0.997727 E OO

$0.998922 \mathrm{E}$ OO
MASS

VELOCITY, G $0.242392 E^{04}$ $\begin{array}{ll}0.159626 \mathrm{E} & 04 \\ 0.120335 \mathrm{E} & 04\end{array}$

$0.752111 E$ O3

$0.432548 \mathrm{E} \quad 03$

0.304031 E 03

0.234476 E 03

$0.190846 E$ O3

$0.160915 \mathrm{E} 03$

$0.139104 E$ O3

$0.122502 E \quad 03$

$0.109441 \mathrm{E} 03$
MASS

VELDCITY, G

$0.401657 \mathrm{E}^{04}$

0.276993 E 04

0.212770 E 04

D. 135527 E 04

D.788628E 03

$0.556717 \mathrm{E} 03$

$0.430321 \mathrm{E} 03$

0.350733 E 03

$0.296002 E \quad 03$

$0.256053 \mathrm{E} 03$

$0.225607 \mathrm{E} 03$

0.201634 E 03

0.191462E 03 
Table 6 (Contd.)

PRESSURE, PSIA $=0.300000 E 02$ SLIP RATIO K $=0.416667 \mathrm{E} 02$

QUALITY, $X$ 1.000000E-02 $0.300000 \mathrm{E}-01$ $0.500000 \mathrm{E}-01$ 1. $000000 \mathrm{E}-01$ $0.200000 \mathrm{E}-00$ $0.300000 \mathrm{E}-00$ 0.400000 E- 00 0.500000 E OO $0.600000 E$ OO 0.700000 E 00 0.800000 E 00 0.900000 E 00 $0.950000 E$ OO

QUALITY,X 1. 0OOODOE-02 $0.300000 E-01$ $0.500000 \mathrm{E}-01$ 1.000000E-01 $0.200000 \mathrm{E}-00$ $0.300000 \mathrm{E}-00$ $0.400000 \mathrm{E}-00$ 0.500000 E OO 0.600000 E 00 0.700000 E 00 0.800000 E 00 0.900000 E 00 0.950000 E OO

QUAL ITY, X 1. 000000E-02 $0.300000 E-01$ $0.500000 \mathrm{E}-01$ 1. $000000 \mathrm{E}-0 \mathrm{I}$ $0.200000 \mathrm{E}-00$ $0.300000 \mathrm{E}-00$ $0.400000 \mathrm{E}-00$ 0.500000 E 00 0.600000 e 00 0.700000 E 0 0.800000 E 00 $0.900000 E$ DO 0.950000 E 00

OUAL I TY, $X$

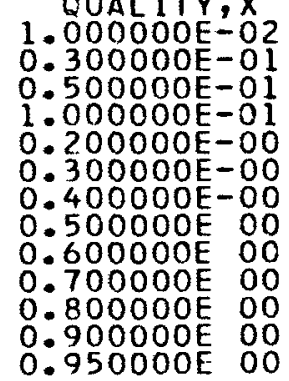

DX/OP
$-0.100791 \mathrm{E}-02$
$-0.938803 \mathrm{E}-03$
$-0.869691 \mathrm{E}-03$
$-0.696911 \mathrm{E}-03$
$-0.351351 \mathrm{E}-03$
$-0.579151 \mathrm{E}-05$
$0.339768 \mathrm{E}-03$
$0.685328 \mathrm{E}-03$
$0.103089 \mathrm{E}-02$
$0.137645 \mathrm{E}-02$
$0.172201 \mathrm{E}-02$
$0.206757 \mathrm{E}-02$
$0.224035 \mathrm{E}-02$

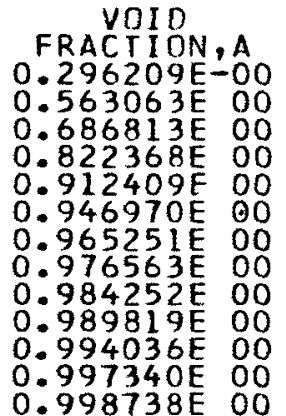
SLIP RATIO K $=0.342675$ E 02
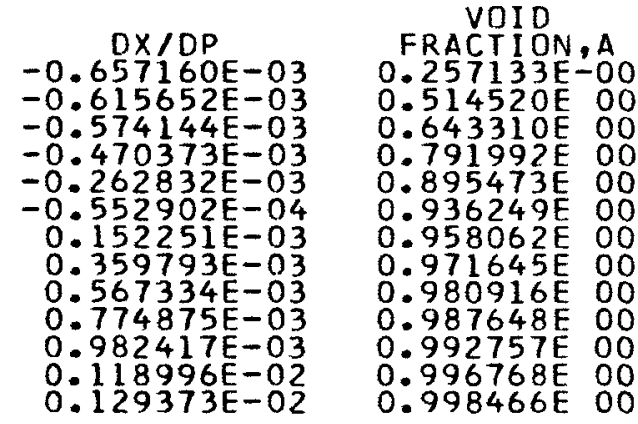

PRESSURE, PSIA $=0.100000 E 03$ SLIP RATIO K $=0.232973 \mathrm{E} \mathrm{O2}$

\begin{tabular}{|c|c|}
\hline $\begin{array}{l}\text { DX/DP } \\
-0.400307 \mathrm{E}-03 \\
-0.378372 \mathrm{E}-03 \\
-0.356438 \mathrm{E}-03 \\
-0.301601 \mathrm{E}-03 \\
-0.191928 \mathrm{E}-03 \\
-0.822549 \mathrm{E}-04 \\
0.274183 \mathrm{E}-04 \\
0.137091 \mathrm{E}-03 \\
0.246765 \mathrm{E}-03 \\
0.356438 \mathrm{E}-03 \\
0.466111 \mathrm{E}-03 \\
0.575784 \mathrm{E}-03 \\
0.630621 \mathrm{E}-03\end{array}$ & 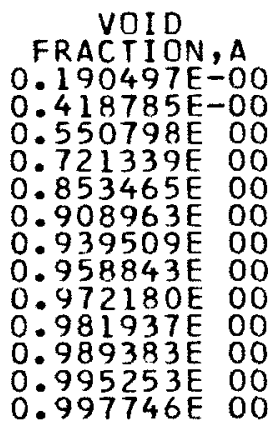 \\
\hline $\begin{array}{l}\text { PRESSURE, PSIA }= \\
\text { SLIP RATIO K }=\end{array}$ & $\begin{array}{l}0.200000 E \\
0.172939 E\end{array}$ \\
\hline $\begin{array}{l}D \times / D P \\
231680 \mathrm{E}-03 \\
219189 \mathrm{E}-03 \\
206698 \mathrm{E}-03 \\
175470 \mathrm{E}-03 \\
113015 \mathrm{E}-03 \\
505591 \mathrm{E}-04 \\
118963 \mathrm{E}-04 \\
743517 \mathrm{E}-04 \\
136807 \mathrm{E}-03 \\
199262 \mathrm{E}-03 \\
261718 \mathrm{E}-03 \\
324173 \mathrm{E}-03 \\
355401 \mathrm{E}-03\end{array}$ & $\begin{array}{l}\text { VOID } \\
\text { ACTION, A } \\
48709 E-00 \\
48476 \mathrm{E}-00 \\
76496 \mathrm{E}-00 \\
57716 \mathrm{E} 00 \\
12153 \mathrm{E} 00 \\
81118 \mathrm{E} 00 \\
20187 \mathrm{E} 00 \\
45337 \mathrm{E} 00 \\
62882 \mathrm{E} 00 \\
75818 \mathrm{E} 00 \\
85750 \mathrm{E} 00 \\
93616 \mathrm{E} 00 \\
96966 \mathrm{E} 00\end{array}$ \\
\hline
\end{tabular}

PRESSURE PSIA $=0.500000 E 02$

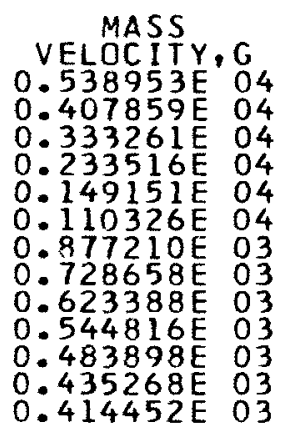

MASS

VELOC ITY, G $0.733845 \mathrm{E} 04$ $0.543409 \mathrm{E} \quad 04$ 0.433667 E 04 $0.289889 \mathrm{E} \quad 04$ $0.175174 \mathrm{E} \quad 04$ 0.125682 E 04 $0.980306 E$ O3 $0.803633 \mathrm{E} \quad 3$ 0.680960 E O3 $0.590798 E$ O3 0.521730 E 03 $0.467126 E \quad 03$ $0.443899 \mathrm{E} \quad 03$

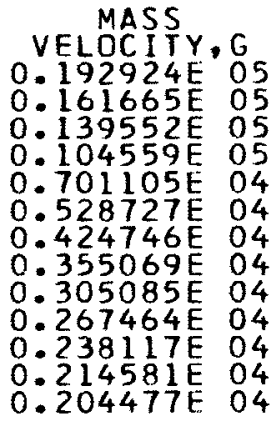


Table 6 (Contd.)

PRESSURE,PSIA $=0.300000 E 03$ SLIP RATIOK $=0.145726 E$ O2

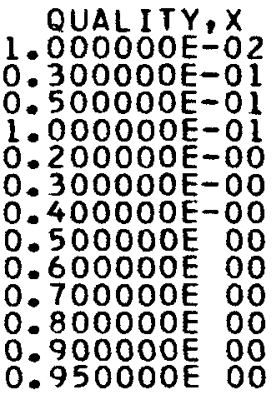

QUAL ITY,X 1. OOOOOOE-02 $0.300000 \mathrm{E}-01$ $0.500000 \mathrm{E}-01$ 1. $000000 \mathrm{E}-01$ $0.200000 E-00$ $0.300000 \mathrm{E}-00$ 0.400000 E- 00 0.500000 E 00 $0.600000 \mathrm{E}$ 00 0.700000 E 00 $0.800000 \mathrm{~F}$ OO O.80000 DO $0.950000 E$ OO

\section{QUALITY, $X$}

1.000000E-02

$0.300000 \mathrm{E}-0 \mathrm{I}$

$0.500000 \mathrm{E}-01$

1.000000E-01

$0.200000 \mathrm{E}-0 \mathrm{O}$

$0.300000 \mathrm{E}-0 \mathrm{O}$

$0.400000 \mathrm{E}-00$

0.500000 E 00

$0.600000 \mathrm{E}$ 00

0.700000 E 00

0.800000 E OO

0.900000 E 00

0.950000 E 00 1. QUALOITY,X $0.300000 \mathrm{E}-01$ $0.500000 \mathrm{E}-01$ 1.000000E-O I 0.200000 E-00 $0.300000 \mathrm{E}-00$ $0.400000 \mathrm{E}-00$ 0.500000 E OO 0.600000 E 00 0.700000 E 00 0.800000 E 00 $0.900000 E$ OD 0.950000 E 00
VOID

FRACTION,A

$-0.167857 \mathrm{E}-03$

$-0.159088 \mathrm{E}-03$

$-0.150319 \mathrm{E}-03$

$-0.128398 \mathrm{E}-03$

$-0.845547 \mathrm{E}-04$

$-0.407115 \mathrm{E}-04$

$0.469748 \mathrm{E}-04$

$0.908180 E-04$

$0.134661 \mathrm{E}-03$

0.128311 E-DO

$0.310677 \mathrm{E}-00$

$0.434063 \mathrm{E}-00$

$0.618201 \mathrm{E}$ OD

0.861982 E 00

$0.906674 \mathrm{E} \quad 00$

$0.935785 E$ OO

$0.956253 \mathrm{E}$ OO

$0.971431 E$ DO

0.983134 E DO

$0.992433 \mathrm{E} 00$

$0.244269 \mathrm{E}-03$

$0.996401 E 00$ PRESSURE, PSIA $=0.500000 E 03$

DX/OP VOID

$-0.114906 \mathrm{E}-03$

$-0.109186 \mathrm{E}-03$

$-0.103466 \mathrm{E}-03$

$-0.891655 \mathrm{E}-04$

$-0.605653 \mathrm{E}-04$

$-0.319650 \mathrm{E}-04$

$-0.336474 E-05$

$0.252355 \mathrm{E}-04$

$0.538358 \mathrm{E}-04$

$0.824361 \mathrm{E}-04$

$0.111036 \mathrm{E}-03$

$0.139637 \mathrm{E}-03$

$0.153937 \mathrm{E}-03$

FRAC TION, A

$0.104962 \mathrm{E}-00$

$0.264202 \mathrm{E}-00$

$0.379285 \mathrm{~F}-00$

$0.563316 \mathrm{E}$ OO

$0.743752 \mathrm{E}$ OO

$0.832654 \mathrm{E}$ OO

$0.885582 \mathrm{E} \quad 00$

$0.920697 E$ EO

$0.945696 \mathrm{E}$ DO

0.964400 E 00

$0.978921 \mathrm{E}$ OO

$0.990520 \mathrm{E}$ OD

$0.995487 E$ OO

PRESSURE,PSIA $=0.700000 E 03$ SLIP RATIO K $=0.996820 E$ OI

DX/DP
$-0.100269 \mathrm{E}-03$
$-0.957432 \mathrm{E}-04$
$-0.912176 \mathrm{E}-04$
$-0.799038 \mathrm{E}-04$
$-0.572762 \mathrm{E}-04$
$-0.346466-04$
$-0.120209 \mathrm{E}-04$
$0.106067 \mathrm{E}-04$
$0.332343 \mathrm{E}-04$
$0.558620 \mathrm{E}-04$
$0.784896 \mathrm{E}-04$
$0.101117 \mathrm{E}-03$
$0.112431 \mathrm{E}-03$

VOID

FRACTION,A

$0.914780 \mathrm{E}-01$

$0.235646 \mathrm{E}-00$

$0.344108 E-00$

$0.525522 \mathrm{E}$ DO

$0.713635 \mathrm{E}$ OO

$0.810322 \mathrm{E} 00$

$0.869204 E$ OO

$0.908827 \mathrm{E} 00$

$0.937313 \mathrm{E}$ OO

$0.958778 \mathrm{E} 00$

$0.975534 \mathrm{E}$ OO

$0.988976 \mathrm{E}$ OO

0.994748 E 00

PRESSURE,PSIA $=0.100000 E 04$ SLIP RATIO K $=$

$0.851697 \mathrm{E} 01$

DX/DP

$-0.776216 E-04$

$-0.743575 \mathrm{E}-04$

$-0.710933 E-04$

$-0.629328 \mathrm{E}-04$

$-0.466119 E-04$

$-0.302910 E-04$

$-0.139701 \mathrm{E}-04$

$0.235075 \mathrm{E}-05$

$0.186716 \mathrm{E}-04$

$0.349925 \mathrm{E}-04$

$0.513134 \mathrm{E}-04$

$0.676343 \mathrm{E}-04$
VOID

FRACTION, A

$0.792152 \mathrm{E}-01$

$0.208492 \mathrm{E}-00$

$0.309517 \mathrm{E}-00$

$0.486213 \mathrm{E}-00$

$0.680434 \mathrm{E} \quad 00$

$0.784952 \mathrm{E} \quad 0$

$0.850254 \mathrm{E} 00$

$0.894925 E \quad 00$

0.927407 E 00

$0.952091 \mathrm{E}$ OO

0.971484 E DO

$0.987122 \mathrm{E} 00$
$0.313165 \mathrm{E}-05$

$0.178504 \mathrm{E}-03$

$0.222347 \mathrm{E}-03$

$0.784629 \mathrm{E}$ OO
MASS

VELOCITY, G

$0.249942 \mathrm{E} 05$

$0.212160 \mathrm{E} 05$

$0.184601 \mathrm{E}$ O5

$0.139770 \mathrm{E} 05$

$0.944433 \mathrm{E} \quad 04$

$0.714243 E \quad 04$

$0.574562 \mathrm{E} 04$

$0.480681 \mathrm{E} 04$

$0.413215 \mathrm{E} 04$

$0.362378 \mathrm{E} \quad 04$

$0.322690 \mathrm{E} 04$

$0.290844 \mathrm{E} \quad 04$

0.277169 E 04

MASS

VELOCITY, G

$0.345408 \mathrm{E} 05$

$0.300167 \mathrm{E} 05$

0.265628 E 05

0.206638 E 05

$0.143433 E$ O5

$0.109949 E$ D

0.891721 E 04

$0.750114 E \quad 04$

$0.647370 \mathrm{E}$ 04

$0.569407 E \quad 04$

$0.508218 E \quad 04$

$0.45891 \mathrm{IE} 04$

$0.437682 \mathrm{E} \quad 04$

MASS

VELOCITY, G

$0.406290 E^{\circ}$

$0.360579 \mathrm{E}$ O5

$0.324367 E$ O5

0.259727 E 05

$0.186226 \mathrm{E}$ O5

0.145338 F 05

$0.119231 \mathrm{E} 05$

0.101097 E 05

$0.877616 \mathrm{E} 04$

0.775395 E 04

$0.694531 \mathrm{E} 04$

$0.628957 \mathrm{E} 04$

$0.600609 \mathrm{E} \quad 04$

MASS

VELOC ITY, G

$0.506323 \mathrm{E}^{\circ} 05$

$0.455039 \mathrm{E}$ O5

$0.413366 \mathrm{E}$ O5

0.336699 E 05

$0.246021 \mathrm{E}$ O5

0.193987 E 05

0.160175 E 05

$0.136421 \mathrm{E} 05$

0.118813 E 05

0.105236 E 05

$0.944467 \mathrm{E} 04$

$0.856656 \mathrm{E} \quad 04$

0.818606E 04 
Table 6 (Contd.)

PRESSURE,PSIA $=0.150000 E$
SLIP RATIO

QUALITY,X

1.000000E-02

$0.300000 \mathrm{E}-01$

$0.500000 E-01$

1. $000000 \mathrm{E}-01$

$0.200000 E-00$

$0.300000 E-00$

$0.400000 \mathrm{E}-00$

0.500000 E 00

0.600000 E 00

0.700000 E 00

0.800000 E 00

0.900000 E 00

0.950000 E 00

QUALITY,X

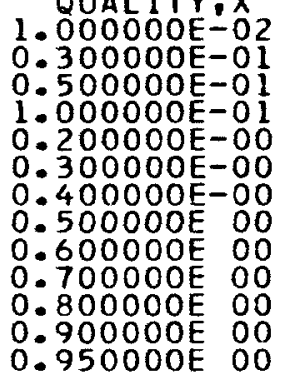

VOID

FRACTION, A $0.674324 \mathrm{E}-01$

$0.181266 \mathrm{E}-00$

$0.273659 \mathrm{E}-00$

$0.443019 \mathrm{E}-00$

0.641530 E DO

$0.754175 \mathrm{E}$ OO

$0.826760 \mathrm{E}$ OO

$0.877429 \mathrm{E} 00$

$0.914805 \mathrm{E} \quad 00$

$0.943513 \mathrm{E} 00$

$0.966255 \mathrm{E} \quad 00$

$0.984716 \mathrm{E}$ OO

$0.992701 E$ OD

$0.514108 \mathrm{E}-04$
$0.575881 \mathrm{E}-04$

PRESSURE, PSIA =

SLIP RATIO $K=$

$D X / D P$

$-0.515583 E-04$

$-0.496682 \mathrm{E}-04$

$-0.477781 \mathrm{E}-04$

$-0.430528 \mathrm{E}-04$

$-0.336022 \mathrm{E}-04$

$-0.241515 \mathrm{E}-04$

$-0.147009 \mathrm{E}-04$

$-0.525034 \mathrm{E}-05$

$0.420027 \mathrm{E}-05$

$0.136509 \mathrm{E}-04$

$0.231015 \mathrm{E}-04$

$0.325521 \mathrm{E}-04$

$0.372774 \mathrm{E}-04$
$0.200000 E 04$

VOID

FRACTION, A

$0.594654 \mathrm{E}-01$

$0.162189 \mathrm{E}-00$

$0.247801 \mathrm{E}-00$

$0.410195 E-00$

0.610109 E 00

$0.728449 \mathrm{E} 0 \mathrm{O}$

$0.806683 \mathrm{E} 00$

$0.862245 E$ OD

$0.903744 \mathrm{E} \quad 00$

$0.935918 \mathrm{E} 00$

$0.961593 \mathrm{E}$ OO

$0.982558 E \quad 00$

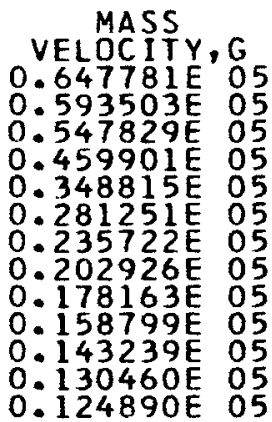

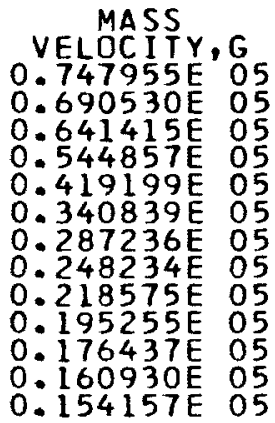


Table 7

COMPUTER INPUT DATA FOR POTASSIUM

$\begin{array}{cc}0.100000 E ~ 01 & 0.500000 E ~ 01 \\ 0.100000 \mathrm{E} 03 & 0.150000 \mathrm{E} 03 \\ 1.000000 \mathrm{E}-02 & 0.300000 \mathrm{E}-01 \\ 0.400000 \mathrm{E}-00 & 0.500000 \mathrm{E} 00 \\ 0.950000 \mathrm{E} 00 & \\ 0.417000 \mathrm{E} 03 & 0.920000 \mathrm{E} 02 \\ 0.580000 \mathrm{E} 01 & 0.401000 \mathrm{E} 01 \\ 0.225000 \mathrm{E}-01 & 0.233600 \mathrm{E}-01 \\ 0.260600 \mathrm{E}-01 & 0.265800 \mathrm{E}-01 \\ 0.123360 \mathrm{E} 01 & 0.116700 \mathrm{E} 01 \\ 0.105060 \mathrm{E} 01 & 0.103640 \mathrm{E} 01 \\ 0.618400 \mathrm{E} 00 & 0.645000 \mathrm{E} 00 \\ 0.708000 \mathrm{E} 00 & 0.719800 \mathrm{E} 00 \\ -0.375000 \mathrm{E} 03 & -0.170000 \mathrm{E} 02 \\ -0.450000 \mathrm{E}-01 & -0.250000 \mathrm{E}-01 \\ -0.400000 \mathrm{E}-01 & -0.9900000 \mathrm{E}-02 \\ -0.350000 \mathrm{E}-03 & -0.220000 \mathrm{E}-03 \\ 0.150000 \mathrm{E}-01 & 0.350000 \mathrm{E}-02 \\ 0.300000 \mathrm{E}-03 & 0.195000 \mathrm{E}-03\end{array}$

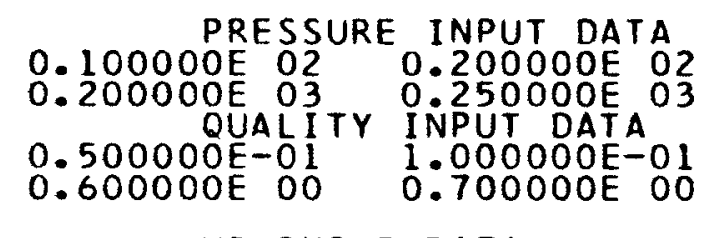

$$
\text { VG INPUT DATA }
$$

$0.470000 E$ O2 $0.244000 E$ O2 $0.315000 \mathrm{E}$ O1 0.260000E O1 0.238400 VE INPUT DATA $\begin{array}{ll}0.238400 \mathrm{E}-01 & 0.243500 \mathrm{E}-01 \\ 0.270000 \mathrm{E}-01 & 0.273500 \mathrm{E}-01\end{array}$ 0.113720 S INPUT DATA 0.11030 OE 01 $\begin{array}{llll}0.113720 E & 01 & 0.111030 E & 01 \\ 0.102730 E & 01 & 0.102020 E & 01\end{array}$ SF INPUT DATA

$0.657100 \mathrm{E}$ OO
$0.728500 \mathrm{E}$ OO 0.670600 E 00 0.728500 O 0.736000 E OO $-0.400000 E^{0} 1$ $-0.125000 \mathrm{E}-\mathrm{O}$
DSGD $-0.390000 E-02$ $-0.162500 \mathrm{E}-03$

$0.185000 \mathrm{E}-02$ $0.167500 E-03$
INPUT DATA -0.875000E-02 INPUT DATA

$-0.192500 E-02$ - $0.125000 \mathrm{E}-03$ INPUT DATA $0.105000 E-02$
$0.150000 E-03$
0.300000 E $02 \quad 0.500000$ E 02

$0.200000 E-00$ $0.800000 E$ OO

$0.300000 E-00$ $0.900000 \mathrm{E} 00$

0.170000 E 02

$0.109000 E 02$

$0.247200 E-01$

$0.252200 E-01$

$0.109440 E$ OI

$0.107550 E$ OI

$0.679500 E 00$

$0.690100 E 00$

$-0.525000 E$ OO

$-0.200000 E-00$

$-0.125000 E-02-0.750000 E-03$

$0.710000 E-03 \quad 0.490000 E-03$ 
Table 8

CRITICAL FLOW DATA FOR POTASSIUM

PRESSURE,PSIA $=0.100000 E 01$

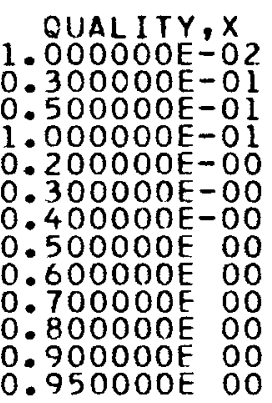

QUAL ITY, $X$

1. 000ODOE-02

$0.300000 \mathrm{E}-01$

$0.500000 \mathrm{E}-0 \mathrm{I}$

1. $000000 E-01$

$0.200000 \mathrm{E}-0 \mathrm{O}$

$0.300000 E-00$

$0.400000 E-00$

0.500000 E 00

0.600000 E 00

0.700000 E 00

0.800000 E 00

0.900000 E 00

0.950000 E OO

QUAL ITY, $X$

1. 000000E-02

0.300000 E-01

$0.500000 \mathrm{E}-0 \mathrm{I}$

$1.000000 \mathrm{E}-01$

$0.200000 \mathrm{E}-00$

$0.300000 \mathrm{E}-00$

$0.400000 E-00$

$0.500000 E$ OO

0.600000 E 00

0.700000 E 00

O. ROODODE OO

0.900000 E OO

0.950000 E 00

QUAL ITY,X

1.000000E-02

$0.300000 \mathrm{E}-01$

$0.500000 \mathrm{E}-01$

$1.000000 \mathrm{E}-01$

$0.200000 \mathrm{E}-0 \mathrm{O}$

$0.300000 \mathrm{E}-00$

$0.400000 \mathrm{E}-0 \mathrm{O}$

0.500000 E 00

0.600000 E 00

0.700000 E 00

0.800000 E 00

0.900000 E 00

$0.950000 \mathrm{E}$ OO

SLIP RATIO $K=0.136137 \mathrm{E}^{0} 3$

$-0.234883 E-01$

$-0.217003 \mathrm{E}-01$

$-0.199122 \mathrm{E}-01$

$-0.154421 \mathrm{E}-01$

$-0.650195 \mathrm{E}-02$

$0.243823 \mathrm{E}-02$

$0.113784 \mathrm{E}-01$

$0.203186 \mathrm{E}-01$

$0.292588 E-01$

$0.381990 \mathrm{E}-01$

$0.471391 \mathrm{E}-01$

$0.560793 \mathrm{E}-01$

PRESSURE, PSIA = SLIP RATIO K =

$D X / D P$

$-0.646552 \mathrm{E}-02$
$-0.598659 \mathrm{E}-02$
$-0.550766 \mathrm{E}-02$
$-0.431034 \mathrm{E}-02$
$-0.191571 \mathrm{E}-02$
$0.478927 \mathrm{E}-03$
$0.287356 \mathrm{E}-02$
$0.526820 \mathrm{E}-02$
$0.766283 \mathrm{E}-02$
$0.100575 \mathrm{E}-01$
$0.124521 \mathrm{E}-01$
$0.148467 \mathrm{E}-01$
$0.160441 \mathrm{E}-01$
$D \times 1 D P$

VOID

$0.578969 \mathrm{~N}, \mathrm{~A}$

$0.808077 \mathrm{E}$ OO

$0.877528 \mathrm{E}$ OO

$0.937990 \mathrm{E}$ OO

$0.971457 \mathrm{E}$ OO

$0.983149 \mathrm{E} \mathrm{OO}$

$0.989102 \mathrm{E}$ 0O

$0.992708 \mathrm{E} \quad 00$

$0.995127 \mathrm{E}$ OO

$0.996862 \mathrm{E}$ 0O

$0.998167 \mathrm{E} 00$

$0.999184 \mathrm{E} \quad 00$

$0.999614 \mathrm{E} 00$

$0.500000 \mathrm{E} 01$

$0.627563 E 02$

PRESSUREIPSIA $=0.100000 E$ O2

$\begin{aligned} & \text { DX/OP } \\ &-0.373360 \mathrm{E}-02 \\ &-0.349406 \mathrm{E}-02 \\ &-0.325453 \mathrm{E}-02 \\ &-0.265570 \mathrm{E}-02 \\ &-0.145803 \mathrm{E}-02 \\ &-0.260362 \mathrm{E}-03 \\ & 0.937305 \mathrm{E}-03 \\ & 0.213497 \mathrm{E}-02 \\ & 0.333264 \mathrm{E}-02 \\ & 0.453031 \mathrm{E}-02 \\ & 0.572797 \mathrm{E}-02 \\ & 0.692564 \mathrm{E}-02 \\ & 0.752447 \mathrm{E}-02\end{aligned}$

VOID

FRACTION, A

$0.309630 \mathrm{E}-00$

$0.578634 \mathrm{E}$ OO

$0.700322 \mathrm{E}$ OO

$0.831465 E$ OO

$0.917358 \mathrm{E}$ DO

0.950073 E OD

$0.967321 \mathrm{~F} 00$

0.977974 E OO

$0.985208 E$ OO

$0.990440 E$ OO

$0.994401 \mathrm{E} 00$

$0.997504 \mathrm{E} 00$

$0.998816 \mathrm{E}$ DO

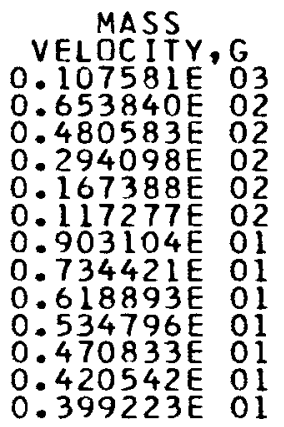

MASS

VELOCI YY, G

$0.366304 E^{\prime} 03$

$0.252970 \mathrm{E} 03$

$0: 196303 \mathrm{E} 03$

$0.127862 \mathrm{E} \quad 03$

0.762680 E 02

$0.545206 \mathrm{E} \quad 2$

$0.424601 E 02$

0.347800 E 02

0.294570 E 02

$0.255491 \mathrm{E} 02$

$0.225576 \mathrm{E}$ O2

$0.201937 \mathrm{E} 02$

0.191885 E 02
PRESSURE,PSIA $=0.200000 E 02$ SLIP RATIO $K=0.316552 \mathrm{E} 02$

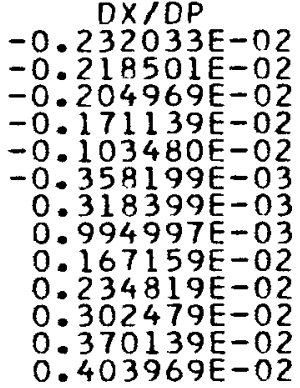

VOID

FRACTION, A

$0.242281 \mathrm{~F}-00$

$0.494701 E-00$

$0.624915 \mathrm{E} \quad 00$

$0.778626 \mathrm{E}$ 0O

$0.887814 E \quad 00$

0.931349 E OO

$0.954758 \mathrm{E}$ OO

0.969377 E OO

0.979374 E 00

$0.986642 \mathrm{E} \quad 00$

$0.992164 \mathrm{E} \quad 00$

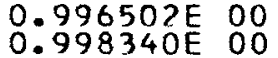

MASS

VELOCITY, G $0.612210 E^{03}$ $0.450308 \mathrm{E} \quad 03$

$0.360751 \mathrm{E} 03$

$0.244672 \mathrm{E} 03$

$0.150903 \mathrm{E} 03$

$0.109534 \mathrm{E} 03$

$0.860635 \mathrm{E} \quad 02$

$0.709071 \mathrm{E} \quad 2$

$0.603019 E$ O2

0.524620 E 02

0.464290 E 02

0.416420 E 02

0.396010 E 02

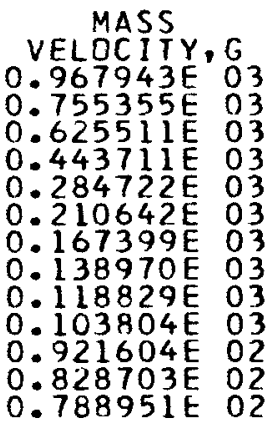


Table 8 (Contd.)

PRESSURE, PSIA $=0.300000 E 02$ SLIP RATIOK $=0.262241 \mathrm{E} 02$

QUAL ITY, X 1. 000000E-02 $0.300000 E-01$ $0.500000 E-01$ 1. $000000 E-01$ $0.200000 E-00$ $0.300000 \mathrm{E}-00$ $0.400000 \mathrm{E}-00$ 0.500000 E 00 0.600000 OO 0.700000 E 00 0.800000 E 00 0.900000 E 00 0.950000 E 00

QUAL ITY, X 1. 00OOOOE -02 0.300000E-01 $0.500000 E-01$ 1. $000000 \mathrm{E}-0 \mathrm{I}$ $0.200000 \mathrm{~F}-00$ $0.300000 \mathrm{E}-00$ $0.400000 E-00$ 0.500000 E DO 0.600000 E 00 $0.700000 \mathrm{~F} 00$ 0.800000 E 00 $0.900000 E$ OO 0.950000 E 00

QUALITY, X 1. OOOOOOE-OZ2 0.3000COE-01 $0.500000 E-01$ 1. $000000 \mathrm{E}-01$ $0.200000 E-00$ $0.300000 \mathrm{E}-00$ $0.400000 E-00$ 0.500000 E 00 0.600000 E OO 0.700000 E 00 0.800000 E OO 0.900000 E 00 0.950000 E 00

QUAL ITY, $X$ $1.000000 E-02$
$0.300000 E-01$
$0.500000 E-01$
$1.000000 E-01$
$0.200000 E-00$
$0.300000 E-00$
$0.400000 E-00$
$0.500000 E$ O0
$0.600000 E$ O0
$0.700000 E$ O0
$0.800000 E$ O0
$0.900000 E$ O0
$0.950000 E$ O0
$\mathrm{D} \times 1 \mathrm{DP}$

$-0.166402 E-02$

$-0.156953 E-02$

$-0.147505 E-02$

$-0.123885 E-02$

$-0.766450 \mathrm{E}-03$

$-0.294047 \mathrm{E}-03$

$0.178356 E-03$

$0.650759 E-03$

$0.112316 \mathrm{E}-02$

$0.159557 \mathrm{E}-02$

$0.206797 E-02$

$0.254037 E-02$

$0.277657 E-02$

PRESSURE PSIA = SLIP RATIO $K=$

$0 \times 10 P$
$-0.123923 E-02$
$-0.117488 \mathrm{E}-02$
$-0.111053 \mathrm{E}-02$
$-0.949663 \mathrm{E}-03$
$-0.627919 \mathrm{E}-03$
$-0.306175 \mathrm{E}-03$
$0.155682 \mathrm{E}-04$
$0.337312 \mathrm{E}-03$
$0.659055 \mathrm{E}-03$
$0.980799 \mathrm{E}-03$
$0.130254 \mathrm{E}-02$
$0.162429 \mathrm{E}-02$
$0.178516 \mathrm{E}-02$

FRAC VOID

0.20947 A

$0.447835 \mathrm{E}-00$

0.579870 E OO

$0.744493 E$ OO

$0.867655 \mathrm{E} \quad 00$

$0.918293 E$ OO

0.945895 E 00

0.963268 E 0

$0.975208 E \quad 00$

0.983920 E 00

0.990557 E 00

$0.995781 \mathrm{E}$ OO 0.997997 E 00

$0.500000 E \quad 02$ $0.207893 \mathrm{E}$ O2

PRESSURE, PSIA $=0.100000 E 03$ SLIP RATIO $K=0.149186 E 02$

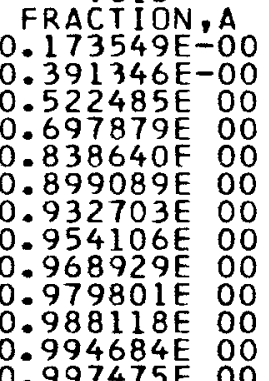

$\mathrm{VOID}$

FRACTION.A

$0.391346 E-00$

$0.522485 E$ OO

0.697879 E 00

$0.899089 \mathrm{E}$ OO

0.932703 E OO

$0.954106 E$ OO

- 97980 L O

$0.988118 E$ OO

0.997475 E

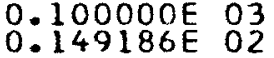

$D X / D P$

$-0.856684 E-03$

$-0.818739 E-03$

$-0.780794 E-03$

$-0.685931 \mathrm{E}-03$

$-0.496205 E-03$

$-0.306480 E-03$

$-0.116754 \mathrm{E}-03$

$0.729714 E-04$

$0.262697 \mathrm{E}-03$

$0.452423 \mathrm{E}-03$

$0.642148 E-03$

$0.831874 E-03$
$0.926737 E-03$

PRESSURE, PSIA = SLIP RATIO K =

$\mathrm{D} \times / \mathrm{DP}$

$-0.602811 E-03$

$-0.576595 E-03$

$-0.550379 E-03$

$-0.484839 \mathrm{E}-03$

$-0.353759 E-03$

$-0.222678 E-03$

$-0.915982 \mathrm{E}-04$

$0.394820 \mathrm{E}-04$

$0.170562 \mathrm{E}-03$

$0.301642 \mathrm{E}-03$

$0.432723 E-03$

$0.563803 \mathrm{E}-03$

$0.629343 \mathrm{E}-03$
VOID

FRACTION, A

O. 130958 E-OO

$0.315724 E-00$

$0.439835 \mathrm{E}-00$

$0.623723 E$ OO

$0.788567 E$ OO

0.864749 E OO

$0.908640 E$ OO

0.937180 E OO

$0.957224 \mathrm{E} \quad 00$

$0.972075 \mathrm{E}$ DO

0.983519 E 00

0.992607 E 00

0.996484 E 00

0.150000 E 03

$0.122827 \mathrm{E}$ OL

VOID

FRACTI DN, A

O. $110374 E-00$

$0.275298 \mathrm{E}-00$

$0.392636 E-00$

$0.577122 \mathrm{E} \quad 00$

$0.754341 \mathrm{E}$ OO

$0.840358 E$ OO

0.891168 E 00

$0.924714 E \quad 00$

$0.948518 E$ OO

$0.966284 E$ OO

$0.980052 E \quad 00$

$\begin{array}{ll}0.991035 \mathrm{E} & 00 \\ 0.995733 \mathrm{E} & 00\end{array}$
MASS

VELDCITY, G

$0.127568 \mathrm{E}^{04}$

$0.101581 \mathrm{E} 04$

$0.849654 \mathrm{E} \quad 03$

$0.609205 E \quad 03$

$0.393266 \mathrm{E} \quad 03$

$0.291427 \mathrm{E} \quad 03$

$0.231744 \mathrm{E} \quad 03$

$0.192439 \mathrm{E} 03$

$0.164570 \mathrm{E} \quad 03$

$0.143769 \mathrm{E} 03$

$0.127645 \mathrm{E} \quad 03$

$0.114778 \mathrm{E} 03$

0.109272 E 03

MASS

VELOC ITY, G $0.170395 E^{\prime} 04$ $0.141102 \mathrm{E} \quad 04$ 0.121090 E 04 $0.902748 \mathrm{E} \quad 03$ $0.605509 \mathrm{E}^{03}$ $0.457633 \mathrm{E} \quad 03$ O. 368370 E 03 0.308447 E 03 0.265378 E 03 $0.232905 E$ O3 $0.207535 \mathrm{E} \quad 03$

$0.187162 \mathrm{E} 03$

0.178409 E 03

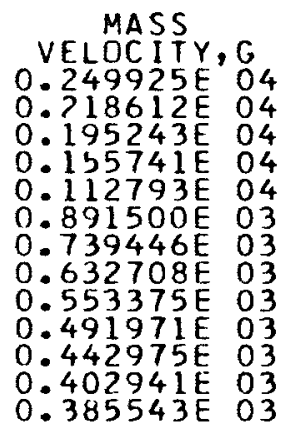

MASS

VELOC ITY, G

$0.330303 E^{04}$ $0.291783 \mathrm{E} \quad 04$ 0.261993 E 04 $0.209934 \mathrm{E} 04$ $0.151634 \mathrm{E} \quad 04$ $0.119205 \mathrm{E} \quad 04$ 0.983737 E 03 $0.838080 \mathrm{E} 03$ $0.730312 \mathrm{E} 03$ $0.647265 \mathrm{E} \quad 03$ $0.581270 \mathrm{E} \quad 3$ $0.527541 \mathrm{E} O 3$ $0.504252 \mathrm{E}$ O3 
Table 8 (Contd.)

PRESSURE, PSIA $=0.200000 E \quad 03$
SLIP RATIO K $=0.108012 \mathrm{E} 02$

QUALITY, $X$

1. $000000 F-02$

$0.300000 E-01$

$0.500000 E-01$

1. $200000 \mathrm{E}-00$

$0.300000 E-00$

$0.400000 E-00$

0.500000 E 00

0.600000 OO

0.700000 E 00

0.800000E OO

0.900000 E 0

0.950000 E 00

QUAL ITY,X 1. 000000E-02

$0.300000 E-01$

$0.500000 E-01$

1. $000000 \mathrm{E}-01$

$0.200000 \mathrm{E}-00$

$0.300000 \mathrm{E}-00$

$0.400000 E-00$

0.500000 E 00

0.600000 E 00

0.700000 E 00

0.800000 E OO

$0.900000 E$ OO

$0.950000 E$ OO
$D \times / D P$

$-0.549531 \mathrm{E}-03$
$-0.527443 \mathrm{E}-03$
$-0.505355 \mathrm{E}-03$
$-0.450134 \mathrm{E}-03$
$-0.339692 \mathrm{E}-03$
$-0.229250 \mathrm{E}-03$
$-0.118809 \mathrm{E}-03$
$-0.836680 \mathrm{E}-05$
$0.102075 \mathrm{E}-03$
$0.212517 \mathrm{E}-03$
$0.322958 \mathrm{E}-03$
$0.433400 \mathrm{E}-03$
$0.488621 \mathrm{E}-03$

FRACTID

FRACTION, A

$0.983708 \mathrm{~F}-01$

$0.250408 \mathrm{E}-00$

$0.362443 \mathrm{E}-00$

$0.545483 \mathrm{E} \quad 00$

0.729752 E 00

$0.822352 \mathrm{E}$ OO

$0.878061 \mathrm{E} \quad 00$

$0.915263 \mathrm{E} \quad 00$

$0.941867 \mathrm{E}$ OO

$0.961836 \mathrm{E} \quad 00$

$0.977378 \mathrm{E} \quad 00$

$0.989818 \mathrm{E} \quad 00$

$0.995151 \mathrm{E}$ OO

PRESSURE, PSIA = SLIP RATIO $K=$

0.250000 E 03 $0.975008 E$ OI

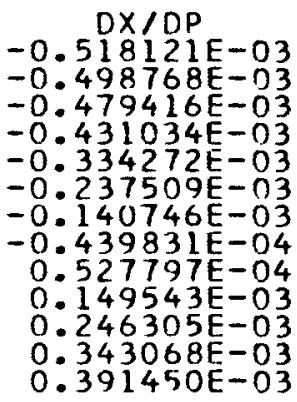

VOID

FRACIION, A

$0.896558 E-01$

$0.231685 E-00$

$0.339132 E-00$

0.520002 E 00

$0.709093 E \quad 00$

0.806898 E 00

$0.866668 E \quad 00$

0.906977 E 00

0.936000 E 00

$0.957895 \mathrm{E}$ OO

$0.975000 E$ OO

$0.988732 \mathrm{E} \quad 00$

$0.994631 \mathrm{E} \quad 00$

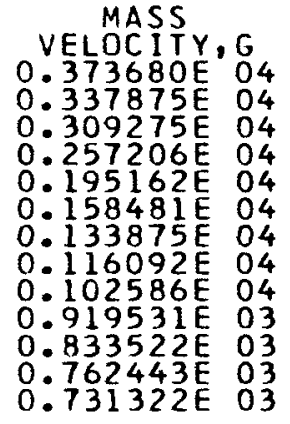

MASS

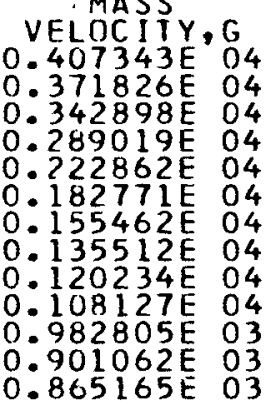


Table 9

COMPUTER INPUT DATA FOR CESIUM

\begin{tabular}{|c|c|c|c|c|}
\hline $\begin{array}{ll}0.100000 E & 01 \\
0: 100000 E & 03\end{array}$ & $\begin{array}{ll}0.500000 E & 01 \\
0.150000 E & 03\end{array}$ & 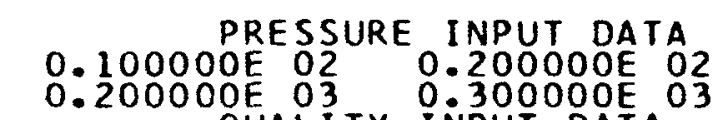 & $0.300000 E 02$ & $0.500000 E 02$ \\
\hline $\begin{array}{l}1.000000 \mathrm{E}-02 \\
0: 400000 \mathrm{E}-00 \\
0.950000 \mathrm{E} 00\end{array}$ & $\begin{array}{l}0.300000 E-01 \\
0.500000 E \text { OO }\end{array}$ & $\begin{array}{l}0.500000 \mathrm{QUAL}-01 \\
0.600000 \mathrm{E}-00\end{array}$ & $\begin{array}{l}0.200000 E-00 \\
0.800000 E 00\end{array}$ & $\begin{array}{l}0.300000 E-00 \\
0.900000 E \text { OD }\end{array}$ \\
\hline $\begin{array}{l}0.100000 E \text { E } \\
0.159000 \mathrm{E}\end{array}$ & $\begin{array}{l}0.231000 E \\
0.111000 E\end{array}$ & $\begin{array}{l}0.124000 \mathrm{VG} \\
0.860000 \mathrm{VE}\end{array}$ & $0.454000 \mathrm{E} \mathrm{O1}$ & $0.293000 E 01$ \\
\hline $\begin{array}{l}0.924200 \mathrm{E}-02 \\
0.991600 \mathrm{E}-02\end{array}$ & $\begin{array}{l}0.940200 \mathrm{E}-02 \\
0.100260 \mathrm{E}-01\end{array}$ & & $0.966100 E-02$ & $0.976000 E-02$ \\
\hline $\begin{array}{l}0.386000 \mathrm{E}-00 \\
0.333900 \mathrm{E}-00\end{array}$ & $\begin{array}{l}0.366300 \mathrm{E}-00 \\
0.330300 \mathrm{E}-00\end{array}$ & $\begin{array}{l}0.358100 \mathrm{~S}-00 \\
0.327900 \mathrm{SE}-00 \\
\text { SF INPU }\end{array}$ & $0.346000 E-00$ & $0.340600 E-00$ \\
\hline $\begin{array}{l}0.216350 E-00 \\
0.245340 \mathrm{E}-00\end{array}$ & $\begin{array}{l}0.224500 \mathrm{E}-00 \\
0.248940 \mathrm{E}-00\end{array}$ & $\begin{array}{l}0.228700 E-00 \\
0.251660 E-00 \\
0.00\end{array}$ & $0.235850 E-00$ & $0.239700 \mathrm{E}-00$ \\
\hline $\begin{array}{l}-0.100000 \mathrm{E} 03 \\
-0: 140000 \mathrm{E}-01\end{array}$ & $\begin{array}{l}-0.400000 E-01 \\
-0.700000 E-02\end{array}$ & $\begin{array}{ll}10000 E & -1 \\
30000 E-02 & -0.3000 \\
0560 P & \text { INPU1750 }\end{array}$ & $-0.135000 E-00$ & $-0.510000 E-01$ \\
\hline $\begin{array}{l}-0.120000 E-01 \\
-0.900000 E-04\end{array}$ & $\begin{array}{l}-0.230000 E-02 \\
-0.600000 E-04\end{array}$ & $\begin{array}{r}-0.550000 E-03 \\
-0.225000 E-04\end{array}$ & $-0.367000 E-03$ & $-0.210000 E-03$ \\
\hline $\begin{array}{l}0.500000 E-02 \\
0.900000 E-04\end{array}$ & $\begin{array}{l}0.120000 \mathrm{E}-02 \\
0.650000 \mathrm{E}-04\end{array}$ & $\begin{array}{l}0.600000 E-03 \\
0.487500 E-04\end{array}$ & $0.225000 E-03$ & $0.160000 E-03$ \\
\hline
\end{tabular}


Table 10

CRITICAL FLOW DATA FOR CESIUM

PRESSURE,PSIA $=0.100000 E$ O1

QUAL ITY, $X$ 1. 000000E-02 $0.300000 E-01$ 1.000000E-0I $0.200000 E-00$ $0.300000 E-00$

$0.400000 E-00$

$0.500000 E$ OO

$0.600000 E$ OO

0.700000 E 00

0.800000 E 00

0.900000 E 00

0.950000 E 00

QUALITY,X

1. 0OOOOOE-O2

$0.300000 \mathrm{E}-01$

$0.500000 E-01$

$1.000000 \mathrm{E}-01$

$0.200000 E-00$

$0.300000 E-00$

$0.400000 \mathrm{E}-00$

0.500000 E 00

0.600000 E 00

0.700000 E 00

0.800000 E 00

$0.950000 \mathrm{E}$ OO

QUALITY,X

1. 00OOOOE-02

$0.300000 \mathrm{E}-01$

$0.500000 E-01$

1. $000000 \mathrm{E}-01$

$0.200000 \mathrm{E}-00$

$0.300000 E-00$

$0.400000 \mathrm{E}-00$

0.500000 E 00

0.600000 E 00

0.700000 E 0

0.800000 E 00

0.900000 E 00
QUALITY, $X$

1. 00000OE-02

$0.300000 \mathrm{~F}-01$

1.000000E-0I

$0.200000 \mathrm{E}-00$

$0.300000 E-00$

$0.400000 E-00$

0.500000 E DO

0.600000 E 00

0.700000 E

0.800000 E 00

0.900000 F DO

0.950000 E 00

VOID

$D \times 1 D P$
$-0.284704 \mathrm{E}-01$
$-0.264663 \mathrm{E}-01$
$-0.244621 \mathrm{E}-01$
$-0.194518 \mathrm{E}-01$
$-0.943118 \mathrm{E}-01$
$0.589449 \mathrm{E}-03$
$0.106101 \mathrm{E}-01$
$0.206307 \mathrm{E}-01$
$0.306513 \mathrm{E}-01$
$0.406720 \mathrm{E}-01$
$0.506926 \mathrm{E}-01$
$0.607132 \mathrm{E}-01$
$0.657235 \mathrm{E}-01$

FRACTION, A

$0.512363 \mathrm{E}^{\circ} 00$

0.762871 IE DO

$0.845554 \mathrm{E} \quad 00$

$0.920368 \mathrm{E}$ OO

$0.962970 \mathrm{E} 00$

$0.978061 \mathrm{E}$ OO

$0.985785 \mathrm{E}$ OO

$0.990478 E$ DO

$0.993632 \mathrm{E} \quad 00$

$0.995897 \mathrm{E} \quad 00$

$0.997602 \mathrm{E}$ OO

$0.998933 \mathrm{E} \quad 00$

$0.999494 \mathrm{E} \quad 00$

PRESSURE, PSIA $=0.500000 E 01$ SLIP RATIO K $=0.495674 \mathrm{E} 02$

$$
\begin{gathered}
D \times / D P \\
-0.821580 \mathrm{E}-02 \\
-0.772214 \mathrm{E}-02 \\
-0.722849 \mathrm{E}-02 \\
-0.599436 \mathrm{E}-02 \\
-0.352609 \mathrm{E}-02 \\
-0.105783 \mathrm{E}-02 \\
0.141044 \mathrm{E}-02 \\
0.387870 \mathrm{E}-02 \\
0.634697 \mathrm{E}-02 \\
0.881523 \mathrm{E}-02 \\
0.112835 \mathrm{E}-01 \\
0.137318 \mathrm{E}-01 \\
0.149859 \mathrm{E}-01
\end{gathered}
$$

PRESSURE, PSIA =

SLIP RATIO $K=$
$D \times / D P$

$-0.449768 \mathrm{E}-02$

$-0.421947 \mathrm{E}-02$

$-0.394127 E-02$

$-0.324575 E-02$

$-0.185471 E-02$

$-0.463679 E-03$

$0.927357 E-03$

$0.231839 E-02$

$0.370943 E-02$

$0.510046 \mathrm{E}-02$

$0.649150 \mathrm{E}-02$

$0.788253 \mathrm{E}-02$
VOID

FRACTION,A

$0.333636 \mathrm{E}-00$

0.605213 E 00

0.722900 E 00

$0.846331 \mathrm{E} 00$

$0.925328 \mathrm{E} \quad 00$

0.955042 E 00

0.970627 E 00

$0.980224 \mathrm{~F} \quad 00$

0.986729 E 00

$0.991428 \mathrm{E}$ 00

$0.994982 \mathrm{E} \quad 00$

0.997763 E 00

$0.998939 \mathrm{E}$ OO

$0.100000 E$ O2

VOID

FRACTION,A

$0.267529 E-00$

0.527927 E 00

$0.655541 \mathrm{E} 00$

$0.800704 \mathrm{E} \quad 00$

$0.900396 \mathrm{E} 00$

0.939382 E OD

0.960169 E 00

0.973089 E 00

$0.981897 \mathrm{E} 00$

0.988286 E 00

$0.993134 E \quad 00$

$0.996937 \mathrm{E} \quad 00$

$0.998547 \mathrm{E} \quad 00$

PRESSURE, PSIA $=0.200000 E 02$

SLIP RATIO K $=0.264319 \mathrm{~F} 02$
$\mathrm{DX} / \mathrm{DP}$

$-0.290708 E-02$

$-0.275362 E-02$

$-0.260017 \mathrm{E}-02$

$-0.221654 E-02$

$-0.144928 \mathrm{E}-02$

$-0.682012 E-03$

$0.852515 E-04$

$0.852515 \mathrm{E}-03$

$0.161978 E-02$

$0.238704 \mathrm{~F}-02$

$0.315431 \mathrm{E}-02$

$0.392157 \mathrm{E}-02$

$0.430520 \mathrm{E}-0$ ?
VOID

FRACIION, A $0.210727 E-00$ $0.449788 E-00$ $0.581791 \mathrm{E}$ OO 0.745991 E OO 0.868559 E 00 $0.918984 \mathrm{E} \quad 00$ $0.946298 E$ OO $0.963546 \mathrm{E}$ OO $0.975398 \mathrm{E} \quad 00$ $0.984045 \mathrm{E} \quad 00$ 0.990630 E 00 $0.995814 \mathrm{E} \quad 00$ 0.998013400
MASS

VELOCITY, G $0.189163 E^{03}$ $0.892438 \mathrm{E} 02$ $0.555033 \mathrm{E}$ 0? $0.319518 E$ O2 $0.224915 \mathrm{E}$ O2 $0.173644 \mathrm{E} \quad 02$ $0.141441 \mathrm{E}$ 02 $0.119326 \mathrm{E}$ O2 $0.103197 \mathrm{E}$ 02 $0.909120 \mathrm{E} 01$ $0.812421 \mathrm{E} \mathrm{O} 1$

$0.771399 \mathrm{E} \mathrm{Ol}$ $0.119605 \mathrm{E} 03$
MASS VELOC IIY, G $0.1 \cup 1903 E^{04}$ $0.778167 \mathrm{E} 03$ $0.636532 \mathrm{E} \quad 03$ $0.444075 \mathrm{E} \quad 03$ $0.280811 \mathrm{E} 03$ $0.206289 \mathrm{E} 03$ 0.163250 E 03 $0.135144 E^{03}$

$0.115324 \mathrm{E} 03$

$0.100588 \mathrm{E} 03$

0.891990 E O2

$0.801306 \mathrm{E} \mathrm{O2}$

$0.762555 \mathrm{E}$ O2
MASS VELOCITY, G $0.155362 \mathrm{E}^{04}$ $0.124986 \mathrm{~F} \quad 04$ $0.105446 E$ E 04

$0.768439 \mathrm{E} \quad 03$

$0.505879 E$ O3

$0.379154 \mathrm{~F} \quad 03$

$0.303739 E$ O3

$0.253536 \mathrm{E} 03$

0.217654 E 03

0.190708 E 03

$0.169720 \mathrm{~F} 03$

$0.152904 E \quad 03$

$0.145690 \mathrm{E} 03$ 
Table 10 (Contd.)

PRESSURE, PSIA $=0.300000 E$ O2 SLIPRATIO K $=0.216779$ E 02

QUALITY,X

1. 000000E-02

$0.300000 E-01$

$0.500000 E-01$

1. $000000 \mathrm{E}-01$

$0.200000 E-00$

$0.300000 E-00$

$0.400000 E-00$

0.500000 E 00

$0.600000 F \quad 00$

0.700000 E OO

0.800000 E 00

0.900000 E OO

0.950000 E OO

QUAL I TY.X 1. OOOOOOF-02 $0.300000 E-01$

$0.500000 E-01$

1.000000E-01

$0.200000 \mathrm{E}-00$

$0.300000 E-00$

0.400000 E-00

$0.500000 E$ OO

$0.600000 E$ OO

0.700000 E OO

$0.800000 \mathrm{~F}$ OO

$0.900000 E$ OO
$0.950000 E$ OO

QUAL I TY, $X$

1. OOOOOOE-O2

0 - $300000 \mathrm{E}-01$

$0.500000 E-01$

1. 000000E-01

$0.200000 E-00$

$0.300000 E-00$

$0.400000 E-00$

0.500000 F 00

0.600000 O 0

$0.700000 E$ OO

$0.800000 E$ OO

$0.900000 E$ OO

QUAL ITY, $X$

1. OODOOOE-02

$0.300000 \mathrm{E}-01$

$0.500000 E-01$

1. $000000 E-01$

$0.200000 E-00$

$0.300000 \mathrm{E}-00$

$0.400000 E-00$

0.500000 E 00

0.600000 E 00

0.700000 E DO

$0.800000 E$ OO

0.900000 E 00

0.950000 E 00
VOID

FRACTION, A

$0.179634 \mathrm{E}-00$

$0.401359 E-00$

$0.532916 E \quad 00$

$0.706629 E \quad 00$

$0.844224 \mathrm{E} \quad 00$

$0.902823 \mathrm{~F} 00$

$0.935283 E \quad 00$

0.970164 E 00

$0.980613 \mathrm{E} \quad 00$

$0.988599 \mathrm{E}$ OO

$0.994901 \mathrm{E}$ OO

O.997578E OO
$0.955904 E$ OO

VELOCITY, G $0.211750 \mathrm{E}^{\circ} \mathrm{O}$ 0.174615 E 04 $0.149491 \mathrm{E} \quad 04$ $0.111117 \mathrm{E} \quad 04$ 0.743758 E 03 $0.561702 E$ E3

$0.451978 E$ O3

0.378378 E 03

0.325503 E 03

0.285649 E 03

$0.25451 \mathrm{AE} 03$

$0.229523 \mathrm{E} 03$

0.218785 E 03

PRESSURE,PSIA $=0.500000 E 02$ SLIP RATIO K $=0.173264 E \quad 02$

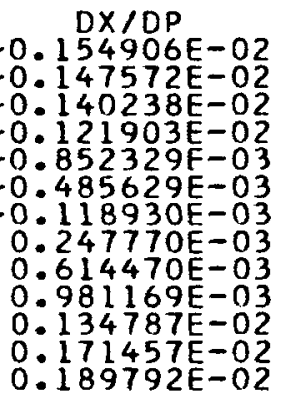

VOID

FRACT ION, A

$0.148947 \mathrm{E}-00$

$0.348903 E-00$

$0.476965 E-00$

0.658138 E 00

0.812439 E 00

$0.881314 \mathrm{E} \quad 00$

$0.920325 \mathrm{E} \quad 00$

$0.945434 \mathrm{~F}$ OO

0.962949 E 00

$0.975862 \mathrm{~F}$ OO

0.985776 E 00

$\begin{array}{ll}0.993628 \mathrm{E} & 00 \\ 0.996972 \mathrm{E} & 00\end{array}$

PRESSURE,PSIA $=0.100000 E 03$ SLIP RATIO $K=0.126628 \mathrm{~K}$ O2

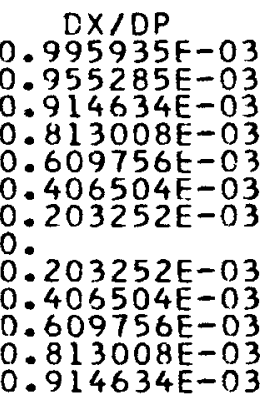

VOID

FRACTION, A

0.113402 E-00

$0.281420 \mathrm{E}-00$

$0.399927 E-00$

$0.584542 E \quad 00$

$0.759945 \mathrm{E} 00$

$0.844405 E$ OO

$0.894089 \mathrm{E} 00$

0.926809 E 00

$0.949986 E$ OO

$0.967263 \mathrm{~F}$ OO

0.980639 E 00

$0.991302 \mathrm{E}$ OO

$0.995861 \mathrm{~F} 00$

PRESSURE, PSIA $=0.150000 E 03$ SLIP RATIO K $=0.105220 \mathrm{E} 02$

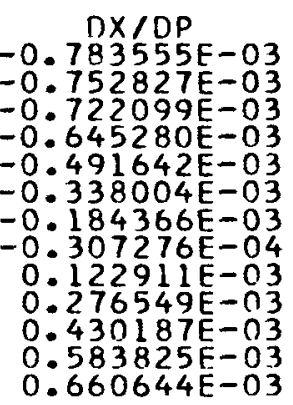

FRACTION, A $0.960719 \mathrm{E}-01$

$0.245523 \mathrm{E}-00$

$0.356412 \mathrm{E}-00$

$0.538981 \mathrm{E}$ OO

$0.724556 \mathrm{E}$ 00

$0.818493 \mathrm{E} \quad 00$

0.875229 E 00

$0.913209 E$ OO

0.940416 E 00

$0.960863 E$ OO

$0.976792 E$ OO

$0.989550 \mathrm{E}$ OO

0.995023 E 00
MASS $0.276042 \mathrm{E}^{\prime} \mathrm{O} 4$ $0.236045 \mathrm{E}$ O4 $0.207292 \mathrm{E} \quad 04$ $0.160603 \mathrm{E} 04$

0.112410 E 04

$0.711794 \mathrm{E}$ O3

$0.602760 \mathrm{E} \quad 03$

0.522991 E 03

0.462020 E 03

$0.413864 \mathrm{E} \quad 03$

$0.374848 \mathrm{E} \quad 3$

$0.357988 \mathrm{E}$ O3

MASS

VELOCITY, $G$

$0.414739 \mathrm{E} 04$

0.367654 E 04

0.331307 E 04

$0.267733 E \quad 04$

$0.196008 \mathrm{E} 04$

$0.155598 \mathrm{E} \quad 04$

$0.129342 \mathrm{E} \quad 04$

$0.110808 \mathrm{E} 04$

$0.969871 \mathrm{E} 03$

$0.862673 E$ O3

$0.777015 \mathrm{E} 03$

$0.706953 \mathrm{E} \quad 03$

$0.676489 E$ O3

MASS

VELDCITY, G $0.520516 E^{\prime} 04$

$0.469378 \mathrm{E} \quad 04$

$0.428429 \mathrm{E} \quad 04$

0.353814 E 04

$0.265240 E$ O4

0.178889 E 04

$0.154198 \mathrm{E} 04$

0.135590 E 04

$0.121039 \mathrm{E} 04$

0.109338 E 04

0.997175 E 03

0.955203 E O3
VELDCITY, G

$0.870363 \mathrm{E} \quad 03$

0.213357 E 04 
Table 10 (Contd.)

PLESSURE,PSIA $=0.200000 E$ O 03

QUAL ITY,X

1. $000000 \mathrm{E}-02$

$0.300000 \mathrm{E}-01$

$0.500000 \mathrm{E}-01$

1.000000E-0 1

$0.200000 E-00$

$0.300000 \mathrm{E}-00$

0.400000 E-00

$0.500000 E$ OO

0.600000 E 00

0.700000 E 00

0.800000 E 00

0.900000 E 0

0.950000 E 00

FRID

FRACTION, A $0.851993 \mathrm{E}-01$

$0.221889 \mathrm{E}-00$

$0.506045 \mathrm{E} 00$

$0.697435 \mathrm{E}$ OD

$0.798043 \mathrm{E} 00$

$0.860078 \mathrm{E}$ OO

$0.902155 \mathrm{E} \quad 00$

$0.932571 E$ OD

0.955583 E OO

$0.973602 \mathrm{E}$ OO

$0.994324 \mathrm{E} \quad 00$

$0.291842 \mathrm{E}-03$

$0.408250 \mathrm{E}-03$

$0.466455 \mathrm{E}-03$
$0.326726 \mathrm{E}-00$

$0.988093 E$ OO

PRESSURE, PSIA =

SLIP RATIO $K=$

$\begin{array}{ll}0.300000 F & 03 \\ 0.777549 E & 01\end{array}$

$D \times / D P$

$-0.481327 \mathrm{E}-03$

$-0.465011 \mathrm{E}-03$

$-0.448695 \mathrm{E}-03$

$-0.407904 \mathrm{E}-03$

$-0.326323 E-03$

$-0.244743 \mathrm{E}-03$

$-0.163162 \mathrm{E}-03$

$-0.815809 \mathrm{E}-04$

$-0.329766 \mathrm{E}-11$

$0.815808 \mathrm{E}-04$

$0.163162 \mathrm{E}-03$

$0.244743 \mathrm{E}-03$

$0.285533 \mathrm{E}-03$
VOIO

FRACTION, A

$0.728210 \mathrm{E}-01$

$0.193860 \mathrm{E}-00$

$0.463503 \mathrm{E}-00$

$0.660311 \mathrm{E} 00$

0.769179 E 00

$0.838284 \mathrm{E} \quad 00$

$0.886046 \mathrm{~F} 00$

$0.921031 \mathrm{E} \mathrm{OO}$

$0.747761 \mathrm{E}$ OD

$0.968849 E$ OO

$0.985911 \mathrm{E}$ OO

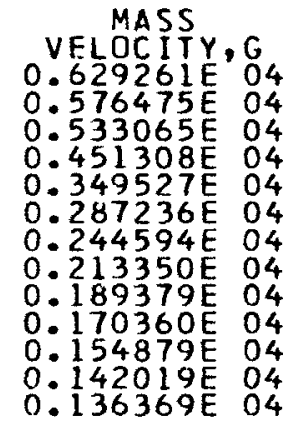

MASS

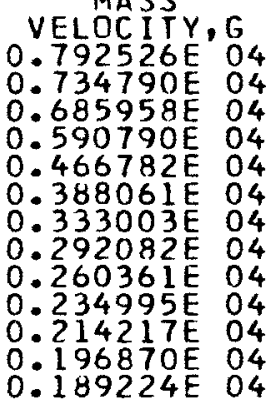


Table 11

COMPUTER INPUT DATA FOR SODIUM

\begin{tabular}{|c|c|c|c|c|}
\hline $\begin{array}{l}0.500000 E-01 \\
0.200000 E \text { O } 2\end{array}$ & $\begin{array}{l}1.000000 E-01 \\
0.300000 \mathrm{E} 02\end{array}$ & 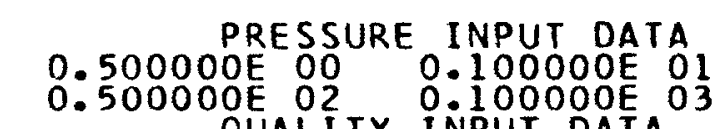 & $\begin{array}{l}0.500000 E \text { O1 } \\
0.150000 \mathrm{E} \\
03\end{array}$ & $0.100000 E 02$ \\
\hline $\begin{array}{l}1.000000 E-02 \\
0.400000 \mathrm{E}-00 \\
0.950000 \mathrm{E} 00\end{array}$ & $\begin{array}{l}0.300000 E-01 \\
0.500000 E \text { OD }\end{array}$ & $\begin{array}{ll}0.500000 \text { QUALITY } & \text { INPUT DATA } \\
0.5000001 & 1.000000 E-01 \\
0.600000 E \text { OD } & 0.700000 E \text { OO }\end{array}$ & $\begin{array}{l}0.200000 E-00 \\
0.800000 E \text { EO }\end{array}$ & $\begin{array}{l}0.300000 E-00 \\
0.900000 E \text { OD }\end{array}$ \\
\hline $\begin{array}{l}0.178000 E \\
0.450000 E\end{array} 02$ & $\begin{array}{l}0.615000 E \\
0.305000 E\end{array}$ & $\begin{array}{ll}O O E E & 03 \\
O O E & 02\end{array}$ & $\begin{array}{ll}\text { OOE } & 03 \\
\text { OOEE O1 }\end{array}$ & $0.845000 \mathrm{~F} 02$ \\
\hline $\begin{array}{l}0.192800 E-01 \\
0.218300 \mathrm{E}-01\end{array}$ & $\begin{array}{l}0.195200 \mathrm{E}-01 \\
0.221100 \mathrm{E}-01\end{array}$ & $0.225100 \mathrm{E}-0$ & $\begin{array}{l}0.209900 \mathrm{E}-01 \\
0.235400 \mathrm{E}-01\end{array}$ & $0.213900 E-01$ \\
\hline $\begin{array}{l}0.226400 E \text { OI } \\
0.179900 \mathrm{E} \text { OI }\end{array}$ & $\begin{array}{l}0.219500 E \text { Ol } \\
0.177300 E \text { OI }\end{array}$ & $0.206900 \mathrm{E}$ OI & $\begin{array}{l}0.189600 E \\
0.167500 \mathrm{E} \\
01\end{array}$ & $0.185100 E 01$ \\
\hline $\begin{array}{l}0.894800 E \text { OO } \\
0.103320 \mathrm{E} \text { O1 }\end{array}$ & $\begin{array}{l}0.906500 E \text { OO } \\
0.104570 \mathrm{E} \text { OI }\end{array}$ & $\begin{array}{l}\text { DAIA } \\
0.956100 E \text { O0 } \\
0.108970 E \text { OI }\end{array}$ & $\begin{array}{l}0.994000 E \text { OO } \\
0.110600 E \text { OI }\end{array}$ & $0.101250 E \quad 01$ \\
\hline $\begin{array}{l}-0.350000 E \text { O6 } \\
-0.200000 \mathrm{E} \text { OI }\end{array}$ & $\begin{array}{l}-0.525000 E \text { OD } \\
-0.104000 \mathrm{E} \text { OI }\end{array}$ & 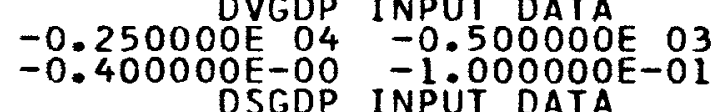 & $\begin{array}{l}-0.350000 E-02 \\
-0.375000 E-01\end{array}$ & $-0.775000 E \quad 01$ \\
\hline $\begin{array}{l}-0.235000 \mathrm{E}-01 \\
-0.320000 \mathrm{E}-02\end{array}$ & $\begin{array}{l}-0.850000 E-00 \\
-0.220000 E-02\end{array}$ & $\begin{array}{l}1.700000 E-01 \\
-0.700000 \\
-0.590000 E-03\end{array}$ & $\begin{array}{l}-0.130000 E-01 \\
-0.400000 E-03\end{array}$ & $-0.700000 E-02$ \\
\hline $\begin{array}{l}0.350000 E-00 \\
0.160000 E-02\end{array}$ & 0.16 & $\begin{array}{l}0.450000 \mathrm{E}-01 \\
0.750000 \mathrm{E}-03\end{array}$ & $\begin{array}{l}\mathrm{OE}-02 \\
\mathrm{OE}-\mathrm{O} 3\end{array}$ & $0.300000 E-02$ \\
\hline
\end{tabular}


Table 12

CRITICAL FLOW DATA FOR SODIUM

PRESSURE, PSIA $=0.500000 E-01$

SLIP RATIO K $=0.960852 E 03$

QUALITY,X

1.000000E-02

$0.300000 \mathrm{E}-01$

$0.500000 \mathrm{E}-01$

$1.000000 \mathrm{E}-01$

$0.200000 \mathrm{E}-0 \mathrm{C}$

$0.300000 \mathrm{E}-0 \mathrm{O}$

$0.400000 \mathrm{E}-00$

$0.500000 \mathrm{E}$ OC

0.600000 E 00

0.700000 E 00

0.800000 E 00

0.900000 E 00

$0.950000 E$ OO

QUAL ITY, $X$

1.000000E-02

$0.300000 \mathrm{E}-01$

$0.500000 \mathrm{E}-01$

1.000000E-OI

$0.200000 E-00$

0.300000 E-OO

$0.400000 \mathrm{E}-0 \mathrm{O}$

$0.500000 E$ DO

0.600000 E 00

0.700000 E 00

0.8000DOE DO

0.900000 E DO

$0.950000 \mathrm{E} 00$

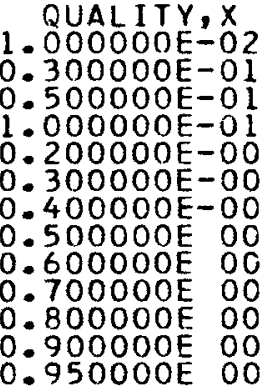

QUAL ITY, $X$

1.000000E-02

$0.300000 \mathrm{E}-01$

$0.500000 \mathrm{E}-01$

1.000000E-0 I

$0.200000 \mathrm{E}-00$

0.300000 E-00

$0.400000 E-00$

0.500000 E DO

0.600000 E 00

0.700000 E 00

0.800000 E 00

0.900000 E 00

0.950000 E 00
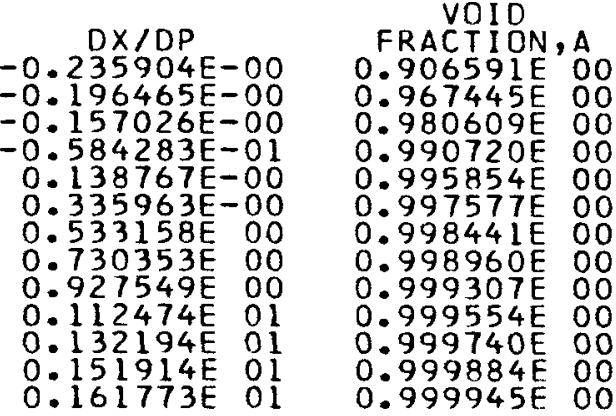

PRESSURE, PSIA $=1.000000 E-01$

SLIP RATIO K $=0.561303 E 03$

$\mathrm{DX} / \mathrm{DP}$

$-0.105929 E-00$

$-0.901436 \mathrm{E}-01$

$-0.506791 \mathrm{E}-01$

$0.282499 \mathrm{E}-01$

$0.107179 E-00$

$0.186108 \mathrm{E}-00$

$0.265037 \mathrm{E}-00$

$0.343966 \mathrm{E}-00$

$0.422895 \mathrm{E}-00$

$0.501824 \mathrm{E} \quad 00$

$0.580753 \mathrm{E}$ 00

$0.620217 E$ OO
$-0.121715 \mathrm{E}-00$

VOID

FRACTION, A

$0.850069 \mathrm{E}$ DO

0.945533 E 00

$0.967259 \mathrm{E}$ OO

$0.984219 \mathrm{E}$ OO

$0.992924 \mathrm{E} \quad 00$

$0.995860 E$ OO

0.997335 E 00

$0.998222 \mathrm{E} \mathrm{OO}$

$0.998814 \mathrm{E}$ OO

$0.999237 \mathrm{E}$ OO

$0.999555 \mathrm{E} \quad 00$

$0.999802 \mathrm{E}$ OO

PRESSURE PSIA $=0.500000 E$ O0

$-0.380762 \mathrm{E}-01$

$-0.344415 \mathrm{E}-01$

$-0.308067 \mathrm{E}-01$

$-0.217199 \mathrm{E}-01$

$-0.354610 \mathrm{E}-02$

$0.146277 \mathrm{E}-01$

$0.328014 \mathrm{E}-01$

$0.509752 \mathrm{E}-01$

$0.691489 \mathrm{E}-01$

$0.873227 \mathrm{E}-01$

$0.105496 \mathrm{E}-00$

$0.123670 \mathrm{E}-00$

O.132757E-OO

PRESSURE, PSIA = SLIP RATIO $K=$

$D X / D P$

$-0.199075 E-01$

$-0.181698 \mathrm{E}-01$

$-0.164321 \mathrm{E}-01$

$-0.120880 \mathrm{E}-01$

$-0.339975 \mathrm{E}-02$

$0.528851 \mathrm{E}-02$

$0.139768 \mathrm{E}-01$

$0.226650 \mathrm{E}-01$

$0.313533 \mathrm{E}-01$

$0.400416 \mathrm{E}-01$

$0.487298 \mathrm{E}-01$

$0.574181 \mathrm{~F}-01$

$0.617622 \mathrm{E}-01$
VOID

FRACIION, A

$0.727902 E^{\circ}$ OO

0.891197 E 0

$0.933061 \mathrm{E} 00$

$0.967134 \mathrm{E}$ OO

$0.985121 \mathrm{E} 00$

$0.991267 \mathrm{E}$ OO

$0.994368 \mathrm{E}$ OO

$0.996238 \mathrm{~F} 00$

0.997489 E 0

$0.998384 \mathrm{E} \quad 00$

0.999057 E OO

$0.999581 E$ OO

$0.999801 E$ OO

0.100000 E 01

$0.185970 \mathrm{E}$ O3

FRACIID

FRACTION,A

$0.652595 \mathrm{E}$ OO

$0.851888 \mathrm{E}$ OD

$0.907304 E$ OO

$0.953839 \mathrm{E}$ OO

$0.978944 \mathrm{E}$ OO

$0.987609 \mathrm{E} 00$

$0.991999 \mathrm{~F}$ OO

$0.996428 \mathrm{E} 00$

$0.997701 \mathrm{E}$ OO

$0.998658 \mathrm{E} 00$

$0.999403 \mathrm{E} \quad 00$

$0.999717 \mathrm{E}$ 00
$0.994652 E$ EO
MASS

VELOCITY, G

$0.594612 E^{\circ}$

0.292419 E 01

$0.198479 \mathrm{E}$ OI

0.111308 E OI

$0.595687 \mathrm{E}$ OO

$0.407060 E-00$

$0.309227 \mathrm{E}-00$

$0.249325 \mathrm{E}-00$

$0.208872 \mathrm{E}-00$

$0.179716 \mathrm{E}-00$

$0.157703 \mathrm{E}-00$

$0.140496 \mathrm{E}-00$

$0.133228 \mathrm{E}-00$

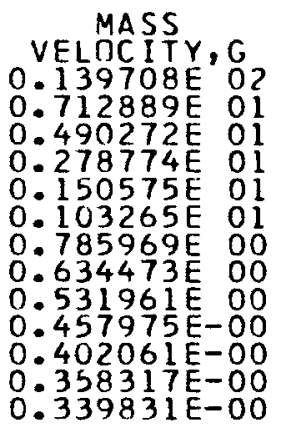

MASS

VELDCITY, G

$0.507178 E^{\circ} 02$

$0.202538 \mathrm{E}$ O2

$0.120160 \mathrm{E}$ 02

$0.669268 E$ E 01

$0.464794 \mathrm{E}$ OI

$0.356196 \mathrm{E}$ OI

$0.288784 \mathrm{E} \quad 01$

$0.242846 \mathrm{E} \quad 1$

$0.209525 \mathrm{E}$ OI

0.184249 E 01

$0.164417 \mathrm{~F}$ OI 
Table 12 (Contd.)

PRESSURE, PSIA $=0.500000 E 01$ SLIPRATIO K $=0.886616 \mathrm{E} \mathrm{O2}$

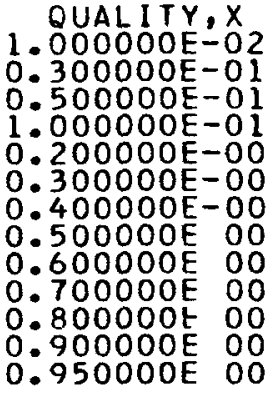

QUALITY, $X$ 1. OOOOOOE-02 $0.300000 \mathrm{E}-01$ $0.500000 E-01$ 1. $000000 E-01$ $0.300000 \mathrm{E}-00$ $0.400000 \mathrm{E}-00$ 0.500COOE OO 0.600000 E 00 0.700000 E 00 0.800000 OO 0.900000 E 00 $0.950000 \mathrm{E} 00$
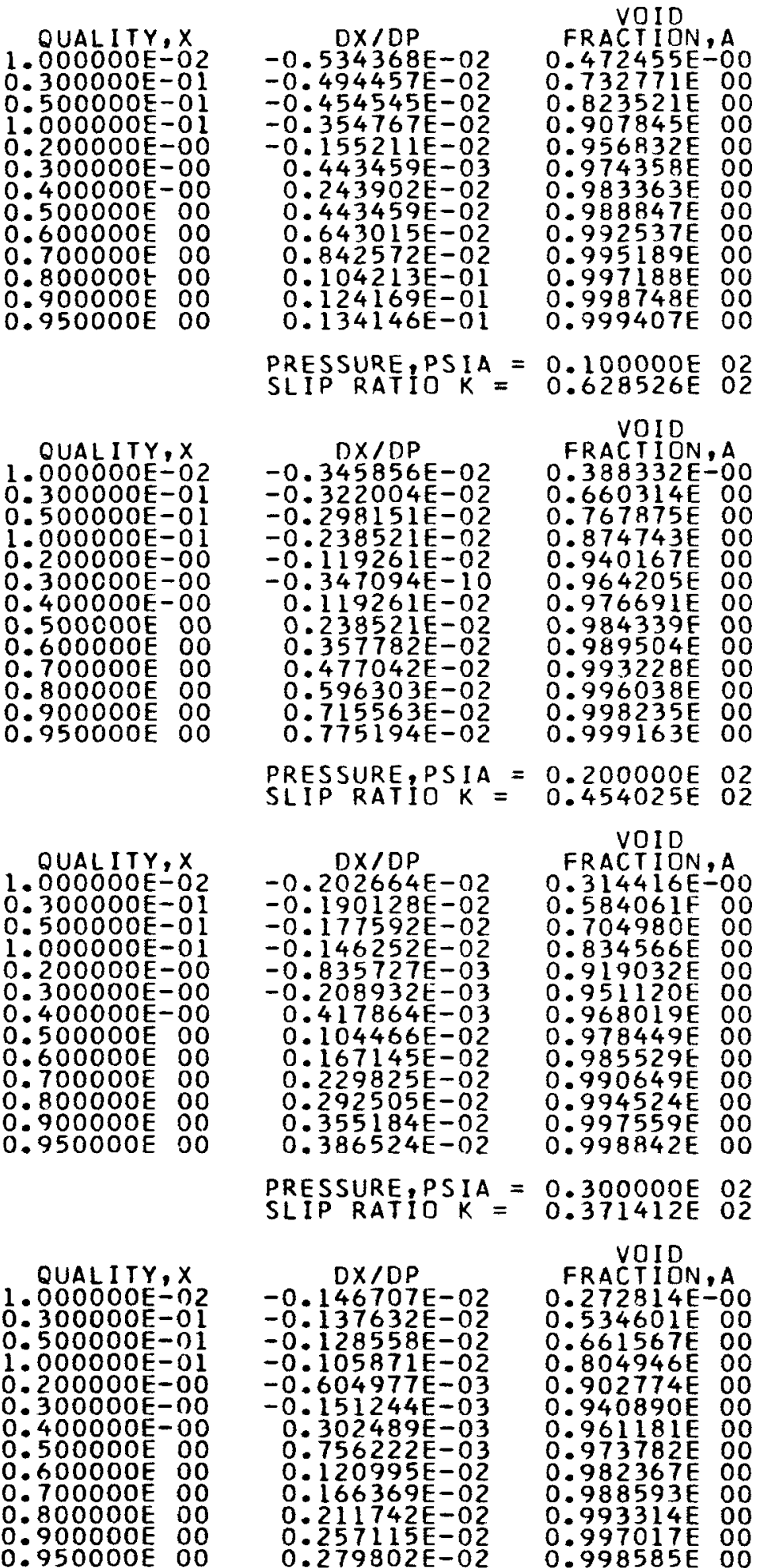

PRESSURE PSIA = SLIP RATIO K =

$0.100000 E \quad 02$

VOIO

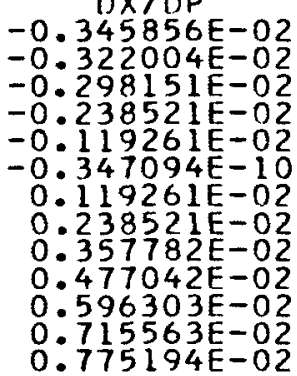

FRACTION A

$0.388332 \mathrm{E}-00$

$0.660314 \mathrm{E} \quad 00$

0.767875 E 00

$0.874743 \mathrm{E}$ OO

$0.940167 \mathrm{E}$ OO

0.964205 E 00

$0.976691 \mathrm{E} 00$

$0.984339 \mathrm{~F} \quad 00$

$0.989504 \mathrm{E} 00$

$0.993228 \mathrm{E}$ OO

$0.996038 \mathrm{E} \quad 00$

$0.998235 \mathrm{E} \quad 00$

$0.999163 E 00$

PRESSURE, PSIA $=0.200000 E 02$ SLIP RATIO K $=0.454025 \mathrm{E} 02$

$D \times 10 P$
$-0.202664 \mathrm{E}-02$
$-0.190128 \mathrm{E}-02$
$-0.177592 \mathrm{E}-02$
$-0.146252 \mathrm{E}-02$
$-0.835727 \mathrm{E}-03$
$-0.208932 \mathrm{E}-03$
$0.417864 \mathrm{E}-03$
$0.104466 \mathrm{E}-02$
$0.167145 \mathrm{E}-02$
$0.229825 \mathrm{E}-02$
$0.292505 \mathrm{E}-02$
$0.355184 \mathrm{E}-02$
$0.386524 \mathrm{E}-02$

FR VOID

FRACTIDN, A

$0.314416 E-00$

$0.584061 \mathrm{~F} 00$

$0.704980 \mathrm{E}$ OD

$0.834566 \mathrm{E}$ OO

$0.919032 \mathrm{E}$ OO

$0.951120 E$ OO

0.968019 E 00

$0.978449 \mathrm{E} 00$

0.985529 E 00

0.990649 E 00

$0.994524 E$ OO

0.997559 E 00

0.998842 E 00

PRESSURE, PSIA $=0.300000 E 02$ SLIP RATIO $K=$

$0.371412 \mathrm{E}$ O2
$D X / D P$

$-0.146707 \mathrm{E}-02$

$-0.137632 \mathrm{E}-02$

$-0.128558 \mathrm{E}-02$

$-0.105871 \mathrm{E}-02$

$-0.604977 \mathrm{E}-03$

$-0.151244 \mathrm{E}-03$

$0.302489 E-03$

$0.756222 \mathrm{E}-03$

$0.120995 \mathrm{E}-02$

$0.166369 E-02$

$0.211742 \mathrm{E}-02$

$0 \cdot 257115 \mathrm{E}-02$
VOID

FRACTIDN, A

$0.272814 \mathrm{E}-00$

$0.534601 \mathrm{E}$ OO

$0.661567 \mathrm{E}$ OO

$0.804946 E$ OO

0.902774 E 00

0.940890 E 00

$0.961181 \mathrm{E} \quad 00$

$0.973782 \mathrm{E}$ OO

0.988593 E 00

$0.993314 \mathrm{E}$ OO

0.997017 E 00
$0.982367 \mathrm{E}$ OO
MASS

VELOCITY, G $0.323482 \mathrm{E}^{0} 3$ 0.206216 E 03

0.153693 E 03

0.950687 E 02

$0.543620 E \quad 02$

$0.381352 \mathrm{E} \quad 02$

$0.293819 \mathrm{E} \quad 2$

$0.239006 E$ O2

$0.201443 E$ O2

0.174090 E 02

$0.153281 \mathrm{E} \quad 02$

$0.136916 \mathrm{E} \quad 02$

0.129979 E 02

MASS

VELOCITY, G

0.525237 E 03

$0.364532 \mathrm{E} 03$

$0.283923 E \quad 03$

$0.186056 \mathrm{E} \quad 03$

$0.111656 \mathrm{E} 03$

0.800611 E 02

0.624647 E 02

0.512286 E 02

$0.434259 \mathrm{E} 02$

$0.376893 \mathrm{E} 02$

$0.332932 \mathrm{E} \quad 02$

$0.298164 \mathrm{E} \quad 02$

0.283370 E 02
VFLOCITY, G

$0.857260 \mathrm{E}^{\circ} \mathrm{O}$

$0.630135 \mathrm{E} 03$

$0.505042 \mathrm{E}$ 03

$0.343100 E$ O3

$0.212116 \mathrm{E} \quad 3$

0.154182 E 03

$0.121254 \mathrm{E} \quad 03$

$0.999627 \mathrm{E} \quad 2$

$0.850508 \mathrm{E} 02$

$0.740191 \mathrm{E} \quad 02$

$0.655251 \mathrm{E} 02$

$0.587823 \mathrm{E}$ O2

0.559065 E 02

MASS

VELDCITY, G $0.113915 E^{\prime} 04$ $0.85551 \mathrm{LE} 03$

$0.691485 \mathrm{E} 03$

$0.472976 \mathrm{E} \quad 3$

$0.292933 E$ O3

$0.212853 \mathrm{E}$ O3

0.167308 E 03

0.137866 E 03

$0.117255 \mathrm{E} \quad 03$

$0.102014 \mathrm{E} 03$

0.902835 E O2

$0.809753 \mathrm{E}$ O2 
Table 12 (Contd.)

PRESSURE,PSIA $=0.500000 E 02$

SLIP RATIO $K=0.292813 E 02$

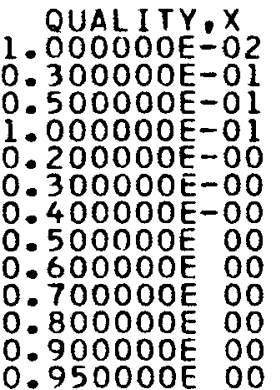

QUALITY,X 1.000000E-02 $0.300000 \mathrm{E}-01$

$0.500000 \mathrm{E}-01$ 1. $000000 \mathrm{E}-01$

$0.200000 \mathrm{E}-00$

$0.300000 \mathrm{E}-00$

$0.400000 \mathrm{E}-00$

$0.500000 E$ OO

$0.600000 E$ OO

0.700000 E 00

$0.800000 E$ OO

$0.900000 E$ OO

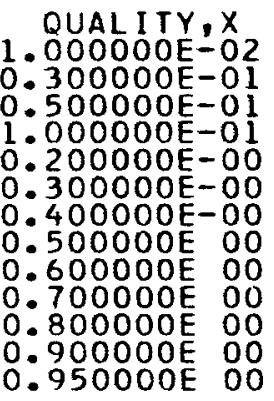

VOID

FRACTION, A

$0.228259 E-00$

$0.475233 \mathrm{E}-00$

$0.606473 \mathrm{E} \quad 00$

$0.764898 \mathrm{E}$ OO

$0.879813 \mathrm{E}$ OD

$0.926195 \mathrm{E}$ 00

0.951269 E DO

$0.966976 \mathrm{E}$ OO

$0.977739 \mathrm{E}$ OO

$0.985575 \mathrm{E}$ OO

$0.991534 \mathrm{E}$ OO

$0.996220 \mathrm{E} 00$

$0.998206 E$ OO

PRESSURE, PSIA =

$0.100000 \mathrm{E} \quad 03$

$-0.681983 E-03$

$-0.648611 \mathrm{E}-03$

$-0.615239 \mathrm{E}-03$

$-0.531810 \mathrm{E}-03$

$-0.364951 E-03$

$-0.198093 \mathrm{E}-03$

$-0.312346 \mathrm{E}-04$

$0.135624 \mathrm{E}-03$

$0.302482 \mathrm{E}-03$

$0.469341 \mathrm{E}-03$

$0.636199 \mathrm{E}-03$

$0.803058 \mathrm{E}-03$

$0.886487 \mathrm{E}-03$

PRESSURE, PS IA =

SLIP RATIO K =

$D \times / D P$

$-0.490580 E-03$

$-0.466467 \mathrm{E}-03$

$-0.442355 E-03$

$-0.382074 \mathrm{E}-03$

$-0.261511 \mathrm{E}-03$

$-0.140949 \mathrm{E}-03$

$-0.203866 E-04$

$0.100176 \mathrm{E}-03$

$0.220738 \mathrm{E}-03$

$0.341301 \mathrm{E}-03$

$0.461863 \mathrm{E}-03$

$0.582425 \mathrm{E}-03$

$0.642706 \mathrm{E}-03$
VOID

FRACTION, A

$0.173542 E-00$

$0.391334 \mathrm{E}-00$

0.522472 E 00

$0.697868 \mathrm{E}$ OO

0.838633 E 00

0.899084 E DO

$0.932700 \mathrm{E} O 0$

$0.954104 \mathrm{E} 00$

$0.968927 \mathrm{E} 00$

$0.979800 E$ OO

0.988117 E OO

$0.994684 \mathrm{E}$ OO

$0.150000 E \quad 03$

$0.172443 \mathrm{E}$ OL

FRACOID

FRACIION,A

$0.347824 \mathrm{E}-00$

$0.475780 \mathrm{E}-00$

$0.657069 \mathrm{E} \mathrm{OO}$

0.811714 E 00

$0.880816 \mathrm{E} 00$

$0.919976 \mathrm{E}$ OO

$0.945188 \mathrm{E}$ OO

0.962779 E OO

0.975750 E OD

0.985710 E 00

0.993598 E 00

$0.996957 \mathrm{E} 00$
MASS

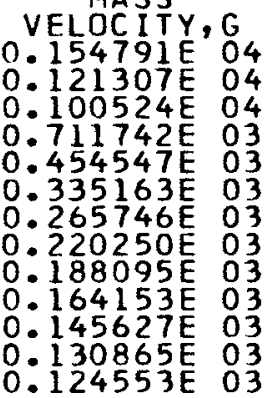

MASS

VELOC IT $Y, G$

$0.239885 \mathrm{E} 04$

$0.198515 \mathrm{E} \quad 04$

$0.170267 \mathrm{E} 04$

$0.126804 \mathrm{E} 04$

$0.849411 E \quad 03$

$0.641468 \mathrm{E} \quad 3$

0.516079 E 03

$0.431967 E \quad 03$

$0.371547 E$ O3

$0.326012 \mathrm{E} 03$

0.290450 E 03

$0.261901 \mathrm{E} \mathrm{D}$

0.249637 E 03

VE MASS

VELOC ITY, G

$0.317004 \mathrm{E}^{04}$

0.271805 E 04

0.239239 E 04

$0.186187 \mathrm{E} \quad 04$

0.131103 E 04

$0.101908 E \quad 04$

0.835726 E 03

0.709167 E 03

0.616300 E 03

$0.545144 E \quad 03$

$0.488831 \mathrm{E} 03$

$0.443131 E$ O3

0.423360 E 03 


\section{ACKNOW LEDGEMENTS}

The author is indebted to the following: J. Zapatka for writing the Fortran Source Program Listing, and D. Quinn for tabulating some of the input data for the IBM-704 computer.

\section{REFERENCES}

1. H. S. Isbin, J. E. Moy, and A. J. R. Cruz, Two-phase, Steam-Water Critical Flow, A.I.Ch.E. Journal, 3, $361-36 \overline{5(1957) .}$

2. D. W. Faletti, Two-phase Critical Flow of Steam-Water Mixtures, Ph.D. Thesis, University of Washington (1959).

3. D. W. Faletti and R. W. Moulton, Two-phase Critical Flow of SteamWater Mixtures, A.I.Ch.E. Journal, 9, 247-253 (1963).

4. H. K. Fauske, Critical Two-phase, Steam-Water Flows, 1961 Proc. Heat Transfer and Fluid Mech. Inst., Stanford University Press, Stanford, p. 79 (1961).

5. H. K. Fauske, Contribution to the Theory of Two-phase, Onecomponent Critical Flow, ANL-6633 (Oct 1962).

6. F. R. Zaloudek, The Low Pressure Critical Discharge of Steam-Water Mixtures from Pipes, HW-68934 Rev. (March 1961).

7. R. James, Steam-Water Critical Flow through Pipes, Proc. of the Institution of Mechanical Engineers, Thermodynamics and Fluid Mechanics Group, 176 (26) (1962).

8. R. S. Pyle, STDY-3, A Program for the Thermal Analysis of a Pressurized Water Nuclear Reactor during Steady-state Operation, Westinghouse Electric Corp, Bettis Atomic Power Laboratory, Pittsburgh, Pa. (June 1961).

9. W. A. Massena, Steam-Water Pressure Drop and Critical Discharge Flow, A Digital Computer Program, HW-65706 (June 1960).

10. A. N. Nahavandi and M. P. Rashevsky, A Digital Computer Program for Critical Flow Discharge of Two-phase Steam-Water Mixtures, CVNA-128 (Feb 1962).

11. W. D. Weatherford, Jr., J. C. Tyler, and P. M. Ku, Properties of Inorganic Energy-Conversion and Heat Transfer Fluids for Space Applications, WADD TR 61-96 (Nov 1961). 


\section{NOMENCLATURE}

G

g

$g_{C}$

k

$\mathrm{p}$

s

$\mathrm{u}$

$\overline{\mathrm{u}}$

$\mathrm{v}$

$\mathrm{x}$

$\alpha$ mass flow rate, $1 b_{m} /(\mathrm{sec})\left(\mathrm{ft}^{2}\right)$

gravitational constant, $32.2 \mathrm{ft} / \mathrm{sec}^{2}$

conversion factor, $32.2 \mathrm{lb} \mathrm{m}^{-\mathrm{ft}} /\left(1 \mathrm{~b}_{\mathrm{f}}\right)\left(\mathrm{sec}^{2}\right)$

$\overline{\mathrm{u}}_{\mathrm{g}} / \overline{\mathrm{u}}_{f}-\operatorname{slip}$ ratio, dimensionless

static pressure, $1 b_{f} / \mathrm{ft}^{2}$

entropy, Btu/1b

velocity, $\mathrm{ft} / \mathrm{sec}$

average velocity, $\mathrm{ft} / \mathrm{sec}$

specific volume, $\mathrm{ft}^{3} / \mathrm{lb}_{\mathrm{m}}$

length in direction of flow, ft

\section{Greek Letters}

void fraction, dimensionless

density, $1 b_{m} / f^{3}$

shear force, $1 b_{f} / f t^{2}$

quality, dimensionless

Subscripts

refers to liquid phase

refers to vapor phase

change in properties between the vapor and liquid state

refers to the ith phase

refers to differentiation under conditions of constant entropy 